Supporting Information

\title{
Reactive Ketimino Radical Acceptors: Intermolecular Alkyl Radical Addition to Imines with a Phenolic Hydroxyl Group
}

Hideto Miyabe, Yousuke Yamaoka, and Yoshiji Takemoto*

Graduate School of Pharmaceutical Sciences, Kyoto University, Yoshida, Sakyo-ku, Kyoto 606-8501, Japan.

takemoto@pharm.kyoto-u.ac.jp

\section{Table of contents}

Experimental procedure and characterization data for $\mathbf{8 a - 2 1 d}, \mathbf{2 3 b}, \mathbf{2 4 b}, \mathbf{2 5 b}-\mathbf{2 9 d}$ and 31-36: S1-S13.

${ }^{1} \mathrm{H}$ and ${ }^{13} \mathrm{C}$ NMR spectrum of all obtained compounds 8a-38: S14-S99.

General. Melting points were taken on a micromelting point apparatus and are uncorrected. ${ }^{1} \mathrm{H}$ and ${ }^{13} \mathrm{C}$ NMR spectra were recorded in $\mathrm{CDCl}_{3}$ at $500 \mathrm{MHz}$, and at $125 \mathrm{MHz}$, respectively. IR spectra were recorded on a Fourier-transfer infrared spectrometer. Mass spectra were obtained by EI or FAB method. Optical rotations were recorded on a polarimeter. Preparative TLC separations were carried out on precoated silica gel plates (E. Merck $60 \mathrm{~F}_{254}$.

Preparation of Aldimines 4-7. Ketimines 4-7 were prepared by the method described in literature. $^{1-4}$

General Procedure for Radical Addition to Aldimines 4-7. To a solution of aldimine 4-7 $(0.5 \mathrm{mmol})$ in $\mathrm{CH}_{2} \mathrm{Cl}_{2}(5 \mathrm{~mL})$ were added $\mathrm{RI}(15 \mathrm{mmol})$ and $\mathrm{Et}_{3} \mathrm{~B}(1.0 \mathrm{M}$ in hexane, 1.25 $\mathrm{mL}, 1.25 \mathrm{mmol}$ ) under nitrogen atmosphere at $20{ }^{\circ} \mathrm{C}$. After being stirred at the same 
temperature for $5 \mathrm{~min}$, the reaction mixture was diluted with saturated $\mathrm{NaHCO}_{3}$ and then extracted with AcOEt. The organic phase was dried over $\mathrm{MgSO}_{4}$ and concentrated at reduced pressure. Purification of the residue by preparative TLC (hexane:AcOEt=10:1-4:1, 2-fold development) afforded $\mathbf{8 a - 1 1 b . ~}$

8a: A colorless crystal. ${ }^{5} \mathrm{mp} 92-94{ }^{\circ} \mathrm{C}$ (AcOEt/hexane). IR $\left(\mathrm{CHCl}_{3}\right) 1707,1497,1457 \mathrm{~cm}^{-1}$. ${ }^{1} \mathrm{H}$ NMR $\left(\mathrm{CDCl}_{3}\right) \delta$ 7.35-7.21 (5H, m), $4.81(1 \mathrm{H}$, br s), $4.54(1 \mathrm{H}$, br s), 1.77 (2H, br m), $1.42(9 \mathrm{H}, \mathrm{s}), 0.89(3 \mathrm{H}, \mathrm{t}, J=7.3 \mathrm{~Hz}) .{ }^{13} \mathrm{C} \mathrm{NMR}\left(\mathrm{CDCl}_{3}\right) \delta 155.4,142.9,128.5,127.1,126.4$, 79.3, 56.3, 29.8, 28.3, 10.6. MS $\left(\mathrm{EI}^{+}\right): \mathrm{m} / \mathrm{z} 236\left(\mathrm{M}+\mathrm{H}^{+}, 23\right), 106$ (100). Anal. Calcd for $\mathrm{C}_{14} \mathrm{H}_{21} \mathrm{NO}_{2}$ : C, 71.46; H, 8.99; N, 5.95. Found: C, 71.20; H, 9.02; N, 5.90.

9a: A colorless crystal. ${ }^{6} \mathrm{mp} 52-54{ }^{\circ} \mathrm{C}$ (AcOEt/hexane). IR $\left(\mathrm{CHCl}_{3}\right) 1718,1509,1457 \mathrm{~cm}^{-1}$.

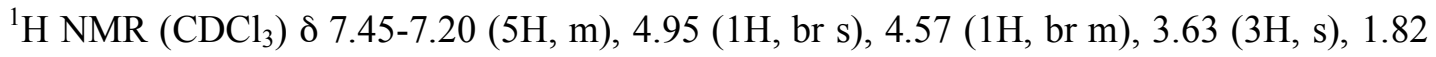
$(2 \mathrm{H}$, br m), 0.89 (3H, t, $J=7.6 \mathrm{~Hz}) .{ }^{13} \mathrm{C} \mathrm{NMR}\left(\mathrm{CDCl}_{3}\right) \delta 156.5,142.6,128.5,127.2,126.4$, 56.8, 51.9, 29.5, 10.5. MS (EI $\left.{ }^{+}\right): \mathrm{m} / \mathrm{z} 193\left(\mathrm{M}^{+}, 5\right), 164(100)$. Anal. Calcd for $\mathrm{C}_{11} \mathrm{H}_{15} \mathrm{NO}_{2}: \mathrm{C}$, 68.37; H, 7.82; N, 7.25. Found: C, 68.36; H, 7.91; N, 7.16.

10a: A colorless oil. ${ }^{7} \mathrm{IR}\left(\mathrm{CHCl}_{3}\right) 1603,1502 \mathrm{~cm}^{-1} .{ }^{1} \mathrm{H} \mathrm{NMR}\left(\mathrm{CDCl}_{3}\right) \delta$ 7.35-7.30 (4H, m), $7.21(1 \mathrm{H}, \mathrm{m}), 7.07(2 \mathrm{H}, \mathrm{br} \mathrm{t}, J=8.0 \mathrm{~Hz}), 6.62(1 \mathrm{H}, \mathrm{t}, J=7.3 \mathrm{~Hz}), 6.50(2 \mathrm{H}, \mathrm{d}, J=8.0 \mathrm{~Hz}), 4.22$ $(1 \mathrm{H}, \mathrm{t}, J=6.7 \mathrm{~Hz}), 4.05(1 \mathrm{H}, \mathrm{br} \mathrm{s}), 1.89-1.75(2 \mathrm{H}, \mathrm{m}), 0.95(3 \mathrm{H}, \mathrm{t}, J=7.6 \mathrm{~Hz}) .{ }^{13} \mathrm{C}$ NMR $\left(\mathrm{CDCl}_{3}\right) \delta 147.5,143.9,129.1,128.5,126.9,126.5,117.2,113.3,59.7,31.6,10.7 . \mathrm{MS}$ $\left(\mathrm{EI}^{+}\right): \mathrm{m} / \mathrm{z} 211\left(\mathrm{M}^{+}, 11\right), 182(100)$. HRMS $\left(\mathrm{EI}^{+}\right)$: calcd for $\mathrm{C}_{15} \mathrm{H}_{17} \mathrm{~N}\left(\mathrm{M}^{+}\right): 211.1361$, Found: 211.1363.

11a: A colorless oil. ${ }^{8} \mathrm{IR}\left(\mathrm{CHCl}_{3}\right) 1517,1449 \mathrm{~cm}^{-1} .{ }^{1} \mathrm{H}$ NMR $\left(\mathrm{CDCl}_{3}\right) \delta$ 7.36-7.17 (5H, m), $6.68(2 \mathrm{H}, \mathrm{m}), 6.54(1 \mathrm{H}$, br t,$J=7.6 \mathrm{~Hz}), 6.43(1 \mathrm{H}$, br d, $J=7.6 \mathrm{~Hz}), 4.75(2 \mathrm{H}$, br s $), 4.20(1 \mathrm{H}$, br t, $J=6.4 \mathrm{~Hz}), 1.93-1.78(2 \mathrm{H}, \mathrm{m}), 0.96(3 \mathrm{H}, \mathrm{t}, J=7.3 \mathrm{~Hz}) .{ }^{13} \mathrm{C} \mathrm{NMR}\left(\mathrm{CDCl}_{3}\right) \delta 143.9$, $143.5,136.2,128.5,126.9,126.6,121.5,117.5,114.2,113.6,60.1,31.4,10.7 . \mathrm{MS}_{\left(\mathrm{EI}^{+}\right) \text {: }}$ m/z $227\left(\mathrm{M}^{+}, 38\right), 198(100)$. HRMS $\left(\mathrm{EI}^{+}\right)$: calcd for $\mathrm{C}_{15} \mathrm{H}_{17} \mathrm{NO}\left(\mathrm{M}^{+}\right)$: 227.1310, Found: 227.1316 .

11b: A colorless oil. IR $\left(\mathrm{CHCl}_{3}\right) 1517,1448 \mathrm{~cm}^{-1} .{ }^{1} \mathrm{H} \mathrm{NMR}\left(\mathrm{CDCl}_{3}\right) \delta$ 7.32-7.17 (5H, m), 6.69-6.63 (2H, m), $6.51(1 \mathrm{H}$, br t, $J=7.6 \mathrm{~Hz}), 6.37(1 \mathrm{H}$, br d, $J=7.6 \mathrm{~Hz}), 4.72(1 \mathrm{H}$, br s), $4.47(1 \mathrm{H}, \mathrm{br} \mathrm{s}), 4.09$ (1H, br d, J=5.8 Hz), $2.07(1 \mathrm{H}, \mathrm{m}), 1.02(3 \mathrm{H}, \mathrm{d}, J=6.7 \mathrm{~Hz}), 0.93(3 \mathrm{H}, \mathrm{d}$, $J=7.0 \mathrm{~Hz}) .{ }^{13} \mathrm{C} \mathrm{NMR}\left(\mathrm{CDCl}_{3}\right) \delta 143.3,142.7,136.6,128.1,127.2,126.8,121.6,117.0$, 


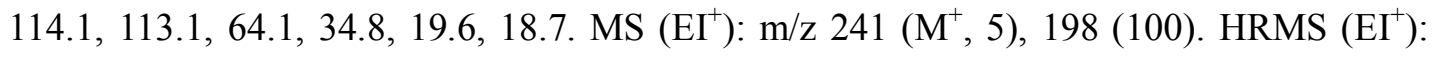
calcd for $\mathrm{C}_{16} \mathrm{H}_{19} \mathrm{NO}\left(\mathrm{M}^{+}\right)$: 241.1467, Found: 241.1462.

Preparation of Ketimines 12, 13, and 15-19. Ketimines 12, 13, and 15-19 were prepared by a modification of the method described in literature. ${ }^{9}$

12: A colorless crystal. ${ }^{9} \mathrm{mp} 96-99{ }^{\circ} \mathrm{C}$ (AcOEt/hexane). IR $\left(\mathrm{CHCl}_{3}\right) 1741 \mathrm{~cm}^{-1} .{ }^{1} \mathrm{H}$ NMR $\left(\mathrm{CDCl}_{3}\right) \delta 7.70(1 \mathrm{H}$, br d, $J=8.0 \mathrm{~Hz}), 7.46(1 \mathrm{H}$, br t,$J=8.0 \mathrm{~Hz}), 7.35(1 \mathrm{H}$, br t, $J=8.0 \mathrm{~Hz})$, $7.27(1 \mathrm{H}$, br d, $J=8.0 \mathrm{~Hz}), 2.57(3 \mathrm{H}, \mathrm{s}) .{ }^{13} \mathrm{C} \mathrm{NMR}\left(\mathrm{CDCl}_{3}\right) \delta 155.2,153.2,146.6,131.1$, 130.5, 128.6, 125.4, 116.4, 21.2. MS (EI $): \mathrm{m} / \mathrm{z} 161\left(\mathrm{M}^{+}, 48\right), 133$ (100). Anal. Calcd for $\mathrm{C}_{9} \mathrm{H}_{7} \mathrm{NO}_{2}$ : C, 67.07; H, 4.38; N, 8.69. Found: C, 67.19; H, 4.41; N, 8.65.

13: A colorless crystal. mp 126-128 ${ }^{\circ} \mathrm{C}$ (AcOEt/hexane). IR $\left(\mathrm{CHCl}_{3}\right) 1739 \mathrm{~cm}^{-1} .{ }^{1} \mathrm{H} \mathrm{NMR}$ $\left(\mathrm{CDCl}_{3}\right) \delta 7.34(1 \mathrm{H}$, br t, $J=7.9 \mathrm{~Hz}), 7.18(1 \mathrm{H}$, br d, $J=7.9 \mathrm{~Hz}), 7.10(1 \mathrm{H}$, br d, $J=7.9 \mathrm{~Hz})$, $2.61(3 \mathrm{H}, \mathrm{s}), 2.58(3 \mathrm{H}, \mathrm{s}) .{ }^{13} \mathrm{C} \mathrm{NMR}\left(\mathrm{CDCl}_{3}\right) \delta 153.5,153.4,146.9,138.0,130.1,129.8$, 126.6, 114.0, 21.3, 16.8. MS $\left(\mathrm{EI}^{+}\right): \mathrm{m} / \mathrm{z} 175\left(\mathrm{M}^{+}, 85\right), 147$ (100). Anal. Calcd for $\mathrm{C}_{10} \mathrm{H}_{9} \mathrm{NO}_{2}$ : C, 68.56; H, 5.18; N, 8.00. Found: C, 68.63; H, 5.39; N, 8.03.

15: A colorless crystal. ${ }^{9} \mathrm{mp} 136-138{ }^{\circ} \mathrm{C}\left(\mathrm{AcOEt} /\right.$ hexane). IR $\left(\mathrm{CHCl}_{3}\right) 1741 \mathrm{~cm}^{-1} .{ }^{1} \mathrm{H} \mathrm{NMR}$ $\left(\mathrm{CDCl}_{3}\right) \delta 7.35(1 \mathrm{H}$, br t, $J=8.3 \mathrm{~Hz}), 7.17(1 \mathrm{H}, \mathrm{br} \mathrm{s}), 6.88(1 \mathrm{H}, \mathrm{br} \mathrm{d}, J=8.3 \mathrm{~Hz}), 6.79(1 \mathrm{H}$, br d, $J=8.3 \mathrm{~Hz}), 2.56(3 \mathrm{H}, \mathrm{s}) .{ }^{13} \mathrm{C} \mathrm{NMR}\left(\mathrm{CDCl}_{3}\right) \delta 153.8,152.7,152.5,147.1,131.8,119.8$, 110.5, 107.2, 21.0. MS $\left(\mathrm{EI}^{+}\right): \mathrm{m} / \mathrm{z} 177\left(\mathrm{M}^{+}, 70\right), 107$ (100). Anal. Calcd for $\mathrm{C}_{9} \mathrm{H}_{7} \mathrm{NO}_{3}: \mathrm{C}$, 61.02; H, 3.98; N, 7.91. Found: C, 60.94; H, 4.12; N, 7.87.

16: A colorless crystal. mp 80-82 ${ }^{\circ} \mathrm{C}$ (AcOEt/hexane). IR $\left(\mathrm{CHCl}_{3}\right) 1763 \mathrm{~cm}^{-1} .{ }^{1} \mathrm{H} \mathrm{NMR}$ $\left(\mathrm{CDCl}_{3}\right) \delta 7.90(1 \mathrm{H}, \mathrm{d}, J=7.9 \mathrm{~Hz}), 7.65(1 \mathrm{H}, \mathrm{t}, J=8.2 \mathrm{~Hz}), 7.45(1 \mathrm{H}, \mathrm{t}, J=7.9 \mathrm{~Hz}), 7.36(1 \mathrm{H}$, d, $J=8.2 \mathrm{~Hz}), 4.51(2 \mathrm{H}, \mathrm{q}, J=7.3 \mathrm{~Hz}), 1.45(3 \mathrm{H}, \mathrm{t}, J=7.3 \mathrm{~Hz}) \cdot{ }^{13} \mathrm{C} \mathrm{NMR}\left(\mathrm{CDCl}_{3}\right) \delta 161.8$, 149.7, 147.2, 145.1, 133.8, 130.5, 130.2, 126.1, 116.7, 63.0, 14.0. $\mathrm{MS}\left(\mathrm{EI}^{+}\right): \mathrm{m} / \mathrm{z} 219\left(\mathrm{M}^{+}\right.$, 22), 91 (100). Anal. Calcd for $\mathrm{C}_{11} \mathrm{H}_{9} \mathrm{NO}_{4}$ : C, 60.27; H, 4.14; N, 6.39. Found: C, 60.25; H, $4.28 ; \mathrm{N}, 6.40$.

17: A colorless crystal. mp 115-117 ${ }^{\circ} \mathrm{C}$ (AcOEt/hexane). IR $\left(\mathrm{CHCl}_{3}\right) 1764,1715 \mathrm{~cm}^{-1} \cdot{ }^{1} \mathrm{H}$ NMR $\left(\mathrm{CDCl}_{3}\right) \delta 7.53(1 \mathrm{H}, \mathrm{t}, J=8.5 \mathrm{~Hz}), 7.38(1 \mathrm{H}, \mathrm{s}), 6.95(1 \mathrm{H}, \mathrm{d}, J=8.5 \mathrm{~Hz}), 6.85(1 \mathrm{H}, \mathrm{d}$, $J=8.5 \mathrm{~Hz}), 4.59(2 \mathrm{H}, \mathrm{q}, J=7.0 \mathrm{~Hz}), 1.44(3 \mathrm{H}, \mathrm{t}, J=7.0 \mathrm{~Hz}) .{ }^{13} \mathrm{C} \mathrm{NMR}\left(\mathrm{CDCl}_{3}\right) \delta 161.6$, 154.6, 150.1, 147.9, 141.3, 135.7, 119.6, 111.5, 107.4, 63.0, 14.0. $\mathrm{MS}\left(\mathrm{EI}^{+}\right): \mathrm{m} / \mathrm{z} 235\left(\mathrm{M}^{+}\right.$, 65), 107 (100). Anal. Calcd for $\mathrm{C}_{11} \mathrm{H}_{9} \mathrm{NO}_{5}$ : C, 56.17; H, 3.86; N, 5.96. Found: C, 56.04; H, 
$3.89 ; \mathrm{N}, 5.90$.

18: A colorless crystal. ${ }^{10} \mathrm{mp} 80-82{ }^{\circ} \mathrm{C}\left(\mathrm{AcOEt} /\right.$ hexane). IR $\left(\mathrm{CHCl}_{3}\right) 1741 \mathrm{~cm}^{-1} .{ }^{1} \mathrm{H}$ NMR $\left(\mathrm{CDCl}_{3}\right) \delta 8.33(2 \mathrm{H}, \mathrm{d}, J=7.3 \mathrm{~Hz}), 7.85(1 \mathrm{H}, \mathrm{d}, J=7.6 \mathrm{~Hz}), 7.57-7.45(4 \mathrm{H}, \mathrm{m}), 7.39(1 \mathrm{H}, \mathrm{t}$, $J=7.6 \mathrm{~Hz}), 7.33(1 \mathrm{H}, \mathrm{d}, J=8.2 \mathrm{~Hz}) .{ }^{13} \mathrm{C} \mathrm{NMR}\left(\mathrm{CDCl}_{3}\right) \delta 152.3,150.9,146.5,134.1,131.7$, $131.4,131.2,129.5,128.4,125.6,116.2$. One peak was missing due to overlapping. MS $\left(\mathrm{EI}^{+}\right): \mathrm{m} / \mathrm{z} 223\left(\mathrm{M}^{+}, 29\right), 195$ (100). Anal. Calcd for $\mathrm{C}_{14} \mathrm{H}_{9} \mathrm{NO}_{2}: \mathrm{C}, 75.33 ; \mathrm{H}, 4.06 ; \mathrm{N}, 6.27$. Found: C, 75.38; H, 4.24; N, 6.31.

19: A colorless crystal. ${ }^{9} \mathrm{mp} 185-187{ }^{\circ} \mathrm{C}\left(\mathrm{AcOEt} /\right.$ hexane). IR $\left(\mathrm{CHCl}_{3}\right) 1741 \mathrm{~cm}^{-1} .{ }^{1} \mathrm{H} \mathrm{NMR}$ $\left(\mathrm{CDCl}_{3}\right) \delta 8.28(2 \mathrm{H}, \mathrm{d}, J=6.7 \mathrm{~Hz}), 7.57-7.45(3 \mathrm{H}, \mathrm{m}), 7.41(1 \mathrm{H}, \mathrm{t}, J=8.2 \mathrm{~Hz}), 7.29(1 \mathrm{H}, \mathrm{s})$, $6.93(1 \mathrm{H}, \mathrm{d}, J=8.2 \mathrm{~Hz}), 6.84(1 \mathrm{H}, \mathrm{d}, J=8.2 \mathrm{~Hz}) .{ }^{13} \mathrm{C} \mathrm{NMR}\left(\mathrm{CDCl}_{3}\right) \delta 153.4,152.8,148.1$,

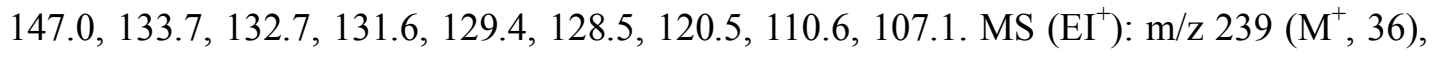
211 (100). Anal. Calcd for $\mathrm{C}_{14} \mathrm{H}_{9} \mathrm{NO}_{3}$ : C, 70.29; H, 3.79; N, 5.86. Found: C, 70.33; H, 3.68; N, 5.86 .

Preparation of Ketimine 14. To a solution of ketimine 13 (500 mg, $2.83 \mathrm{mmol})$ in DMF $(10 \mathrm{~mL})$ were added $\mathrm{K}_{2} \mathrm{CO}_{3}(780 \mathrm{mg}, 5.66 \mathrm{mmol})$ and $\mathrm{MeI}(0.53 \mathrm{~mL}, 8.49 \mathrm{mmol})$ under argon atmosphere at $20{ }^{\circ} \mathrm{C}$. After being stirred at the same temperature for $10 \mathrm{~h}$, the reaction mixture was diluted with $\mathrm{H}_{2} \mathrm{O}$ and then extracted with $\mathrm{Et}_{2} \mathrm{O}$. The organic phase was dried over $\mathrm{MgSO}_{4}$ and concentrated at reduced pressure. Purification of the residue by chromatography (hexame:AcOEt=3:1) afforded $14(216 \mathrm{mg}, 40 \%)$ as a colorless crystal. mp 145-147 ${ }^{\circ} \mathrm{C}$ (AcOEt/hexane). IR $\left(\mathrm{CHCl}_{3}\right) 1741 \mathrm{~cm}^{-1} .{ }^{1} \mathrm{H}$ NMR $\left(\mathrm{CDCl}_{3}\right) \delta 7.41(1 \mathrm{H}, \mathrm{br} \mathrm{t}$, $J=8.2 \mathrm{~Hz}), 6.89(1 \mathrm{H}$, br d, $J=8.2 \mathrm{~Hz}), 6.85(1 \mathrm{H}, \mathrm{br} \mathrm{d}, J=8.2 \mathrm{~Hz}), 4.03(3 \mathrm{H}, \mathrm{s}), 2.56(3 \mathrm{H}, \mathrm{s})$. ${ }^{13} \mathrm{C} \mathrm{NMR}\left(\mathrm{CDCl}_{3}\right) \delta 155.6,153.4,153.3,147.9,131.1,121.8,108.6,107.1,56.6,21.4 . \mathrm{MS}$ $\left(\mathrm{EI}^{+}\right): \mathrm{m} / \mathrm{z} 191\left(\mathrm{M}^{+}, 82\right), 134$ (100). Anal. Calcd for $\mathrm{C}_{10} \mathrm{H}_{9} \mathrm{NO}_{3}: \mathrm{C}, 62.82 ; \mathrm{H}, 4.74 ; \mathrm{N}, 7.33$. Found: C, 62.98; H, 4.80; N, 7.35.

General Procedure for Radical Addition to Ketimines 12-14 in the presence of Yb(OTf) 3 . To a solution of ketimine 12-14 $(0.5 \mathrm{mmol})$ in $\mathrm{THF}-\mathrm{CH}_{2} \mathrm{Cl}_{2}(1: 2, \mathrm{v} / \mathrm{v}, 3 \mathrm{~mL})$ were added $\mathrm{Yb}(\mathrm{OTf})_{3}(310 \mathrm{mg}, 0.5 \mathrm{mmol}), \mathrm{RI}(7.5 \mathrm{mmol})$ and $\mathrm{Et}_{3} \mathrm{~B}(1.0 \mathrm{M}$ in hexane, 1.25 $\mathrm{mL}, 1.25 \mathrm{mmol}$ ) under nitrogen atmosphere at $20{ }^{\circ} \mathrm{C}$. After being stirred at the same temperature for $5 \mathrm{~min}, \mathrm{Et}_{3} \mathrm{~B}(1.0 \mathrm{M}$ in hexane, $1.25 \mathrm{~mL}, 1.25 \mathrm{mmol})$ added to the reaction mixture. After being stirred at the same temperature for $5 \mathrm{~min}, \mathrm{Et}_{3} \mathrm{~B}$ (1.0 $\mathrm{M}$ in hexane, 1.25 
$\mathrm{mL}, 1.25 \mathrm{mmol}$ ) added to the reaction mixture. After being stirred for 5-50 min, the reaction mixture was diluted with saturated $\mathrm{NaHCO}_{3}$ and then extracted with AcOEt. The organic phase was dried over $\mathrm{MgSO}_{4}$ and concentrated at reduced pressure. Purification of the residue by preparative TLC (hexane:AcOEt=4:1, 2-fold development) afforded

\section{0a-24b.}

20a: A colorless crystal. mp $45-48{ }^{\circ} \mathrm{C}$ (hexane). IR $\left(\mathrm{CHCl}_{3}\right) 1731 \mathrm{~cm}^{-1} .{ }^{1} \mathrm{H}$ NMR $\left(\mathrm{CDCl}_{3}\right)$

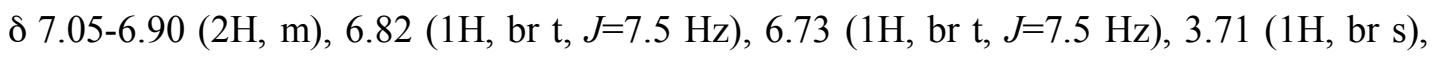
$1.85(1 \mathrm{H}, \mathrm{m}), 1.68(1 \mathrm{H}, \mathrm{m}), 1.45(3 \mathrm{H}, \mathrm{s}), 0.97(3 \mathrm{H}, \mathrm{t}, J=7.3 \mathrm{~Hz}) .{ }^{13} \mathrm{C} \mathrm{NMR}\left(\mathrm{CDCl}_{3}\right) \delta 168.6$,

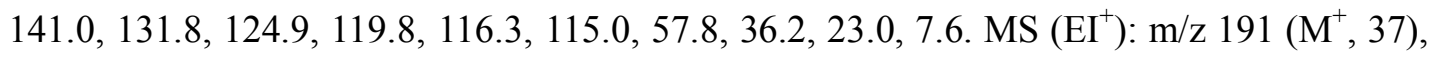
134 (100). Anal. Calcd for $\mathrm{C}_{11} \mathrm{H}_{13} \mathrm{NO}_{2}: \mathrm{C}, 69.09 ; \mathrm{H}, 6.85 ; \mathrm{N}, 7.32$. Found: $\mathrm{C}, 68.89 ; \mathrm{H}$, $6.75 ; \mathrm{N}, 7.16$.

20b: A colorless crystal. mp 117-119 ${ }^{\circ} \mathrm{C}$ (AcOEt/hexane). IR $\left(\mathrm{CHCl}_{3}\right) 1762 \mathrm{~cm}^{-1} .{ }^{1} \mathrm{H}$ NMR $\left(\mathrm{CDCl}_{3}\right) \delta$ 7.00-6.85 $(2 \mathrm{H}, \mathrm{m}), 6.80(1 \mathrm{H}$, br t, $J=7.9 \mathrm{~Hz}), 6.72(1 \mathrm{H}, \mathrm{br} \mathrm{d}, J=7.9 \mathrm{~Hz}), 3.88(1 \mathrm{H}$, br s), $2.12(1 \mathrm{H}, \mathrm{m}), 1.40(3 \mathrm{H}, \mathrm{s}), 0.96(3 \mathrm{H}, \mathrm{d}, J=7.0 \mathrm{~Hz}), 0.92(3 \mathrm{H}, \mathrm{d}, J=7.0 \mathrm{~Hz}) .{ }^{13} \mathrm{C} \mathrm{NMR}$ $\left(\mathrm{CDCl}_{3}\right) \delta 168.2,141.1,131.5,124.9,119.7,116.2,114.8,60.4,31.7,19.2,16.8,15.8 . \mathrm{MS}$ $\left(\mathrm{EI}^{+}\right): \mathrm{m} / \mathrm{z} 205\left(\mathrm{M}^{+}, 17\right), 162$ (100). Anal. Calcd for $\mathrm{C}_{12} \mathrm{H}_{15} \mathrm{NO}_{2}: \mathrm{C}, 70.22 ; \mathrm{H}, 7.37 ; \mathrm{N}, 6.82$. Found: C, 70.18; H, 7.46; N, 6.72 .

20c: A colorless crystal. mp 120-123 ${ }^{\circ} \mathrm{C}$ (AcOEt/hexane). IR $\left(\mathrm{CHCl}_{3}\right) 1763 \mathrm{~cm}^{-1} .{ }^{1} \mathrm{H}$ NMR $\left(\mathrm{CDCl}_{3}\right) \delta$ 7.00-6.85 $(2 \mathrm{H}, \mathrm{m}), 6.80(1 \mathrm{H}, \mathrm{br} \mathrm{t}, J=7.6 \mathrm{~Hz}), 6.69(1 \mathrm{H}, \mathrm{br} \mathrm{d}, J=7.6 \mathrm{~Hz}), 3.83(1 \mathrm{H}$, br s), 1.83-1.60 (6H, m), $1.42(3 \mathrm{H}, \mathrm{s}), 1.23-1.02(5 \mathrm{H}, \mathrm{m}) .{ }^{13} \mathrm{C} \mathrm{NMR}\left(\mathrm{CDCl}_{3}\right) \delta 168.1,141.0$, 131.5, 124.9, 119.5, 116.2, 114.6, 60.5, 42.2, 27.0, 26.1 (3C), 25.9, 20.4. MS (EI $\left.{ }^{+}\right): \mathrm{m} / \mathrm{z} 245$ $\left(\mathrm{M}^{+}, 15\right), 162$ (100). Anal. Calcd for $\mathrm{C}_{15} \mathrm{H}_{19} \mathrm{NO}_{2}$ : C, 73.44; H, 7.81; N, 5.71. Found: $\mathrm{C}$, 73.47; H, 7.90; N, 5.72.

20d: A colorless crystal. mp 137-140 ${ }^{\circ} \mathrm{C}$ (AcOEt/hexane). IR $\left(\mathrm{CHCl}_{3}\right) 1754 \mathrm{~cm}^{-1} .{ }^{1} \mathrm{H} \mathrm{NMR}$ $\left(\mathrm{CDCl}_{3}\right) \delta$ 6.95-6.85 (2H, m), $6.70(1 \mathrm{H}$, br t, $J=7.9 \mathrm{~Hz}), 6.64(1 \mathrm{H}$, br d, $J=7.9 \mathrm{~Hz}), 3.90(1 \mathrm{H}$, br s), $1.51(3 \mathrm{H}, \mathrm{s}), 1.03(9 \mathrm{H}, \mathrm{s}) .{ }^{13} \mathrm{C} \mathrm{NMR}\left(\mathrm{CDCl}_{3}\right) \delta 167.5,140.4,132.5,125.0,118.4$,

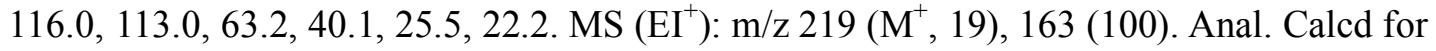
$\mathrm{C}_{13} \mathrm{H}_{17} \mathrm{NO}_{2}$ : C, 71.21; H, 7.81; N, 6.39. Found: C, 71.15; H, 7.85; N, 6.41.

21a: A colorless crystal. mp 49-51 ${ }^{\circ} \mathrm{C}$ (AcOEt/hexane). IR $\left(\mathrm{CHCl}_{3}\right) 1762 \mathrm{~cm}^{-1} .{ }^{1} \mathrm{H}$ NMR $\left(\mathrm{CDCl}_{3}\right) \delta 6.87$ (2H, br d, $\left.J=7.6 \mathrm{~Hz}\right), 6.74$ (1H, br t, $\left.J=7.6 \mathrm{~Hz}\right), 3.54$ (1H, br s), $2.19(3 \mathrm{H}, \mathrm{s})$, 
$1.82(1 \mathrm{H}, \mathrm{m}), 1.66(1 \mathrm{H}, \mathrm{m}), 1.47(3 \mathrm{H}, \mathrm{s}), 0.95(3 \mathrm{H}, \mathrm{t}, J=7.6 \mathrm{~Hz}) .{ }^{13} \mathrm{C} \mathrm{NMR}\left(\mathrm{CDCl}_{3}\right) \delta$ 168.7,

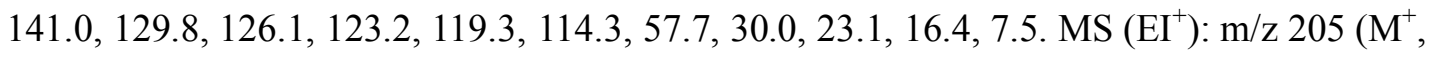
40), 148 (100). Anal. Calcd for $\mathrm{C}_{12} \mathrm{H}_{15} \mathrm{NO}_{2}$ : C, 70.22; H, 7.37; N, 6.82. Found: C, 70.25; H, $7.35 ; \mathrm{N}, 6.79$.

21b: A colorless crystal. mp 83-85 ${ }^{\circ} \mathrm{C}$ (AcOEt/hexane). IR $\left(\mathrm{CHCl}_{3}\right) 1761 \mathrm{~cm}^{-1} .{ }^{1} \mathrm{H}$ NMR $\left(\mathrm{CDCl}_{3}\right) \delta$ 6.88-6.83 (2H, m), $6.73(1 \mathrm{H}$, br t, $J=7.9 \mathrm{~Hz}), 3.67(1 \mathrm{H}, \mathrm{br} \mathrm{s}), 2.20(3 \mathrm{H}, \mathrm{s}), 2.06$ $(1 \mathrm{H}, \mathrm{m}), 1.43(3 \mathrm{H}, \mathrm{s}), 0.96(3 \mathrm{H}, \mathrm{d}, J=6.7 \mathrm{~Hz}), 0.89(3 \mathrm{H}, \mathrm{d}, J=6.7 \mathrm{~Hz}) .{ }^{13} \mathrm{C} \mathrm{NMR}\left(\mathrm{CDCl}_{3}\right)$ $\delta 168.2,141.1,129.5,126.1,122.8,119.1,114.0,60.4,31.4,18.9,16.9,16.3,15.7 . \mathrm{MS}$ $\left(\mathrm{EI}^{+}\right): \mathrm{m} / \mathrm{z} 219\left(\mathrm{M}^{+}, 45\right), 69(100)$. Anal. Calcd for $\mathrm{C}_{13} \mathrm{H}_{17} \mathrm{NO}_{2}: \mathrm{C}, 71.21 ; \mathrm{H}, 7.81 ; \mathrm{N}, 6.39$. Found: C, 71.24; H, 7.88; N, 6.32 .

21d: A colorless crystal. mp 99-102 ${ }^{\circ} \mathrm{C}$ (AcOEt/hexane). IR $\left(\mathrm{CHCl}_{3}\right) 1753 \mathrm{~cm}^{-1} .{ }^{1} \mathrm{H}$ NMR $\left(\mathrm{CDCl}_{3}\right) \delta 6.82(1 \mathrm{H}$, br d, $J=7.9 \mathrm{~Hz}), 6.78(1 \mathrm{H}$, br d, $J=7.9 \mathrm{~Hz}), 6.63(1 \mathrm{H}$, br t, $J=7.9 \mathrm{~Hz})$, $3.64(1 \mathrm{H}, \mathrm{br} \mathrm{s}), 2.19(3 \mathrm{H}, \mathrm{s}), 1.54(3 \mathrm{H}, \mathrm{s}), 1.01(9 \mathrm{H}, \mathrm{s}) .{ }^{13} \mathrm{C} \mathrm{NMR}\left(\mathrm{CDCl}_{3}\right) \delta 167.5,140.3$,

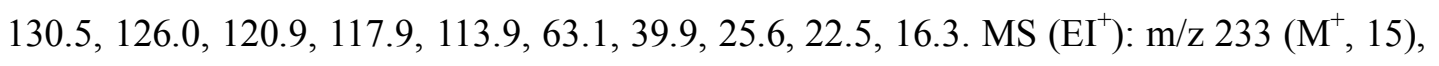
176 (100). Anal. Calcd for $\mathrm{C}_{14} \mathrm{H}_{19} \mathrm{NO}_{2}$ : C, 72.07; H, 8.21; N, 6.00. Found: C, 71.88; H, 8.26; N, 5.92.

23b: A colorless oil. IR $\left(\mathrm{CHCl}_{3}\right) 1758 \mathrm{~cm}^{-1} .{ }^{1} \mathrm{H} \mathrm{NMR}\left(\mathrm{CDCl}_{3}\right) \delta 6.96(1 \mathrm{H}$, br d, $J=6.7 \mathrm{~Hz})$, 6.97-6.80 (2H, m), $3.81(1 \mathrm{H}$, br s), $2.88(1 \mathrm{H}, \mathrm{m}), 2.08(1 \mathrm{H}, \mathrm{m}), 1.42(3 \mathrm{H}, \mathrm{s}), 1.31(3 \mathrm{H}, \mathrm{d}$, $J=6.7 \mathrm{~Hz}), 1.23(3 \mathrm{H}, \mathrm{d}, J=6.7 \mathrm{~Hz}), 0.96(3 \mathrm{H}, \mathrm{d}, J=6.7 \mathrm{~Hz}), 0.90(3 \mathrm{H}, \mathrm{d}, J=6.7 \mathrm{~Hz}) .{ }^{13} \mathrm{C}$ $\operatorname{NMR}\left(\mathrm{CDCl}_{3}\right) \delta 168.3,141.5,133.9,128.2,121.2,119.6,113.8,60.1,31.3,27.3,22.8$, 22.3, 18.9, 16.9, 15.9. MS (EI'): $\mathrm{m} / \mathrm{z} 247\left(\mathrm{M}^{+}, 7\right), 204$ (100). HRMS (EI $\left.{ }^{+}\right)$: calcd for $\mathrm{C}_{15} \mathrm{H}_{21} \mathrm{NO}_{2}\left(\mathrm{M}^{+}\right)$: 247.1572, Found: 247.1579.

24b: A colorless solid. IR $\left(\mathrm{CHCl}_{3}\right) 1760 \mathrm{~cm}^{-1} .{ }^{1} \mathrm{H}$ NMR $\left(\mathrm{CDCl}_{3}\right) \delta 6.84(1 \mathrm{H}, \mathrm{s}), 6.83(1 \mathrm{H}$, br d, $J=7.0 \mathrm{~Hz}), 6.64(1 \mathrm{H}$, br d, $J=7.0 \mathrm{~Hz}), 3.75(1 \mathrm{H}, \mathrm{br} \mathrm{s}), 2.82(1 \mathrm{H}, \mathrm{m}), 2.11(1 \mathrm{H}, \mathrm{m}), 1.38$ $(3 \mathrm{H}, \mathrm{s}), 1.21(6 \mathrm{H}, \mathrm{d}, J=7.0 \mathrm{~Hz}), 0.95(3 \mathrm{H}, \mathrm{d}, J=7.0 \mathrm{~Hz}), 0.92(3 \mathrm{H}, \mathrm{d}, J=7.0 \mathrm{~Hz}) .{ }^{13} \mathrm{C} \mathrm{NMR}$ $\left(\mathrm{CDCl}_{3}\right) \delta 168.5,141.1,141.0,129.0,122.7,114.7,114.0,60.4,33.2,31.3,24.0,19.0,16.8$, 15.7. One peak was missing due to overlapping. $\mathrm{MS}\left(\mathrm{EI}^{+}\right): \mathrm{m} / \mathrm{z} 247\left(\mathrm{M}^{+}, 15\right), 204(100)$. HRMS (EI ${ }^{+}$): calcd for $\mathrm{C}_{15} \mathrm{H}_{21} \mathrm{NO}_{2}\left(\mathrm{M}^{+}\right)$: 247.1572, Found: 247.1568 .

General Procedure for Radical Addition to Ketimine 15 in $\mathrm{CH}_{2} \mathrm{Cl}_{2}$ at $20{ }^{\circ} \mathrm{C}$. To a solution of ketimine 15 (100 mg, $0.57 \mathrm{mmol})$ in $\mathrm{CH}_{2} \mathrm{Cl}_{2}(4 \mathrm{~mL})$ were added $\mathrm{Bu}_{3} \mathrm{SnH}(0.46$ 
$\mathrm{mL}, 1.7 \mathrm{mmol})$, RI $(17 \mathrm{mmol})$ and $\mathrm{Et}_{3} \mathrm{~B}(1.0 \mathrm{M}$ in hexane, $1.43 \mathrm{~mL}, 1.43 \mathrm{mmol})$ under nitrogen atmosphere at $20^{\circ} \mathrm{C}$. After being stirred at the same temperature for $5 \mathrm{~min}$, the reaction mixture was diluted with saturated $\mathrm{NaHCO}_{3}$ and then extracted with AcOEt. The organic phase was dried over $\mathrm{MgSO}_{4}$ and concentrated at reduced pressure. Purification of the residue by preparative TLC (hexane:AcOEt=4:1, 2-fold development) afforded 25a-25d.

25b: A colorless crystal. mp 99-101 ${ }^{\circ} \mathrm{C}\left(\mathrm{Et}_{2} \mathrm{O} /\right.$ hexane). IR $\left(\mathrm{CHCl}_{3}\right) 1762 \mathrm{~cm}^{-1} .{ }^{1} \mathrm{H}$ NMR $\left(\mathrm{CDCl}_{3}\right) \delta 6.70(1 \mathrm{H}$, br t, $J=8.0 \mathrm{~Hz}), 6.64-6.57(2 \mathrm{H}, \mathrm{m}), 5.63(1 \mathrm{H}, \mathrm{br} \mathrm{s}), 4.01(1 \mathrm{H}, \mathrm{br} \mathrm{s})$, $2.08(1 \mathrm{H}, \mathrm{m}), 1.42(3 \mathrm{H}, \mathrm{s}), 0.96(3 \mathrm{H}, \mathrm{d}, J=6.7 \mathrm{~Hz}), 0.92(3 \mathrm{H}, \mathrm{d}, J=7.0 \mathrm{~Hz}) .{ }^{13} \mathrm{C}$ NMR $\left(\mathrm{CDCl}_{3}\right) \delta 168.9,144.3,142.6,120.0,119.6,111.0,108.7,60.4,31.3,18.8,16.9,15.7 . \mathrm{MS}$ $\left(\mathrm{EI}^{+}\right): \mathrm{m} / \mathrm{z} 221\left(\mathrm{M}^{+}, 7\right), 178(100)$. Anal. Calcd for $\mathrm{C}_{12} \mathrm{H}_{15} \mathrm{NO}_{3}: \mathrm{C}, 65.14 ; \mathrm{H}, 6.83 ; \mathrm{N}, 6.33$. Found: C, 65.12; H, 6.82; N, 6.22.

25c: A colorless crystal. mp 110-112 ${ }^{\circ} \mathrm{C}\left(\mathrm{Et}_{2} \mathrm{O} /\right.$ hexane $)$. IR $\left(\mathrm{CHCl}_{3}\right) 1761 \mathrm{~cm}^{-1} .{ }^{1} \mathrm{H}$ NMR $\left(\mathrm{CDCl}_{3}\right) \delta 6.69(1 \mathrm{H}$, br t, $J=8.0 \mathrm{~Hz}), 6.62(1 \mathrm{H}$, br d, $J=8.0 \mathrm{~Hz}), 6.57(1 \mathrm{H}$, br d, $J=8.0 \mathrm{~Hz})$, $5.35(1 \mathrm{H}, \mathrm{s}), 3.99(1 \mathrm{H}, \mathrm{s}), 1.65-1.67(6 \mathrm{H}, \mathrm{m}), 1.44(3 \mathrm{H}, \mathrm{s}), 1.45-1.05(5 \mathrm{H}, \mathrm{m}) .{ }^{13} \mathrm{C} \mathrm{NMR}$ $\left(\mathrm{CDCl}_{3}\right) \delta 169.2,144.0,142.1,120.2,119.1,111.1,108.5,60.5,41.8,27.1,26.1$ (2C), 26.0, 25.7, 20.1. MS (EI $\left.{ }^{+}\right): \mathrm{m} / \mathrm{z} 261\left(\mathrm{M}^{+}, 4\right), 178$ (100). Anal. Calcd for $\mathrm{C}_{15} \mathrm{H}_{19} \mathrm{NO}_{3}: \mathrm{C}, 68.94 ; \mathrm{H}$, 7.33; N, 5.36. Found: C, 68.88; H, 7.42; N, 5.33.

25d: A colorless crystal. mp 118-119 ${ }^{\circ} \mathrm{C}\left(\mathrm{Et}_{2} \mathrm{O} /\right.$ hexane). IR $\left(\mathrm{CHCl}_{3}\right) 1752 \mathrm{~cm}^{-1} .{ }^{1} \mathrm{H}$ NMR

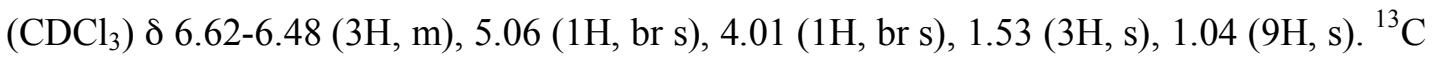
NMR $\left(\mathrm{CDCl}_{3}\right) \delta 168.2,142.2,141.4,121.1,117.8,111.0,108.7,63.1,39.9,25.6,22.3 . \mathrm{MS}$ $\left(\mathrm{EI}^{+}\right): \mathrm{m} / \mathrm{z} 235\left(\mathrm{M}^{+}, 5\right), 178$ (100). Anal. Calcd for $\mathrm{C}_{13} \mathrm{H}_{17} \mathrm{NO}_{3}: \mathrm{C}, 66.36 ; \mathrm{H}, 7.28 ; \mathrm{N}, 5.95$. Found: C, 66.38; H, 7.14; N, 5.92.

General Procedure for Radical Addition to Ketimines 16, 18, and 19 in the presence of $\mathrm{Zn}(\mathrm{OTf})_{2}$. To a solution of ketimine $\mathbf{1 6 - 1 9}(0.5 \mathrm{mmol})$ in $\mathrm{CH}_{2} \mathrm{Cl}_{2}(5 \mathrm{~mL})$ were added $\mathrm{Zn}(\mathrm{OTf})_{2}(182 \mathrm{mg}, 0.5 \mathrm{mmol}), \mathrm{RI}(7.5 \mathrm{mmol}), \mathrm{Bu}_{3} \mathrm{SnH}(0.40 \mathrm{~mL}, 1.5 \mathrm{mmol}$ or none $)$ and $\mathrm{Et}_{3} \mathrm{~B}(1.0 \mathrm{M}$ in hexane, $1.25 \mathrm{~mL}, 1.25 \mathrm{mmol})$ under nitrogen atmosphere at $20{ }^{\circ} \mathrm{C}$. After being stirred for 5-60 min, the reaction mixture was diluted with saturated $\mathrm{NaHCO}_{3}$ and then extracted with AcOEt. The organic phase was dried over $\mathrm{MgSO}_{4}$ and concentrated at reduced pressure. Purification of the residue by preparative TLC (hexane:AcOEt=4:1, 
2-fold development) afforded 26a-b, 28a and 29a-b.

General Procedure for Radical Addition to Ketimines 17 and 19. To a solution of ketimines 17 or $19(0.5 \mathrm{mmol})$ in $\mathrm{CH}_{2} \mathrm{Cl}_{2}(5 \mathrm{~mL})$ were added RI (7.5 mmol), Bu 3 SnH (0.40 $\mathrm{mL}, 1.5 \mathrm{mmol}$ or none) and $\mathrm{Et}_{3} \mathrm{~B}(1.0 \mathrm{M}$ in hexane, $1.25 \mathrm{~mL}, 1.25 \mathrm{mmol})$ under nitrogen atmosphere at $20{ }^{\circ} \mathrm{C}$. After being stirred at the same temperature for $5 \mathrm{~min}$, the reaction mixture was diluted with saturated $\mathrm{NaHCO}_{3}$ and then extracted with AcOEt. The organic phase was dried over $\mathrm{MgSO}_{4}$ and concentrated at reduced pressure. Purification of the residue by preparative TLC (hexane:AcOEt=4:1, 2-fold development) afforded 27a-d and 29d.

26a: A colorless oil. IR $\left(\mathrm{CHCl}_{3}\right)$ 1777, $1738 \mathrm{~cm}^{-1} .{ }^{1} \mathrm{H} \mathrm{NMR}\left(\mathrm{CDCl}_{3}\right) \delta 7.01(2 \mathrm{H}, \mathrm{m}), 6.87$ $(2 \mathrm{H}, \mathrm{m}), 4.42(1 \mathrm{H}, \mathrm{br} \mathrm{s}), 4.13(2 \mathrm{H}, \mathrm{m}), 2.26(1 \mathrm{H}, \mathrm{m}), 2.10(1 \mathrm{H}, \mathrm{m}), 1.13(3 \mathrm{H}, \mathrm{t}, J=7.0 \mathrm{~Hz})$, $1.12(3 \mathrm{H}, \mathrm{t}, J=7.6 \mathrm{~Hz}) .{ }^{13} \mathrm{C} \mathrm{NMR}\left(\mathrm{CDCl}_{3}\right) \delta 170.2,163.4,140.8,132.1,125.1,121.1,116.7$,

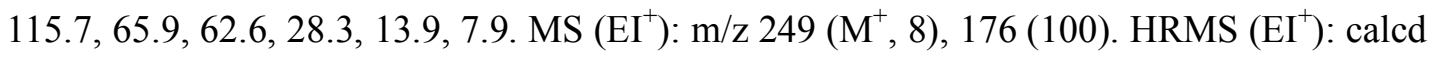
for $\mathrm{C}_{13} \mathrm{H}_{15} \mathrm{NO}_{4}\left(\mathrm{M}^{+}\right)$: 249.1001, Found: 249.0992 .

26b: A colorless oil. IR $\left(\mathrm{CHCl}_{3}\right)$ 1775, $1737 \mathrm{~cm}^{-1} .{ }^{1} \mathrm{H} \mathrm{NMR}\left(\mathrm{CDCl}_{3}\right) \delta$ 7.03-6.97 (2H, m), 6.89-6.82 (2H, m), $4.35(1 \mathrm{H}, \mathrm{br} \mathrm{s}), 4.22-4.08(2 \mathrm{H}, \mathrm{m}), 2.68(1 \mathrm{H}, \mathrm{m}), 1.18(3 \mathrm{H}, \mathrm{d}, J=6.7 \mathrm{~Hz})$, $1.16(3 \mathrm{H}, \mathrm{t}, J=7.1 \mathrm{~Hz}), 1.07(3 \mathrm{H}, \mathrm{d}, J=7.0 \mathrm{~Hz}) \cdot{ }^{13} \mathrm{C} \mathrm{NMR}\left(\mathrm{CDCl}_{3}\right) \delta 169.7,162.8,140.2$,

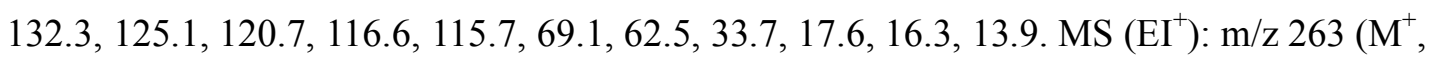
10), 190 (100). HRMS (EI $\left.{ }^{+}\right)$: calcd for $\mathrm{C}_{14} \mathrm{H}_{17} \mathrm{NO}_{4}\left(\mathrm{M}^{+}\right)$: 263.1158, Found: 263.1151.

27a: A colorless crystal. mp 92-94 ${ }^{\circ} \mathrm{C}$ (AcOEt/hexane). IR $\left(\mathrm{CHCl}_{3}\right) 1773,1739 \mathrm{~cm}^{-1} .{ }^{1} \mathrm{H}$ $\operatorname{NMR}\left(\mathrm{CDCl}_{3}\right) \delta 6.72(1 \mathrm{H}$, br t,$J=7.9 \mathrm{~Hz}), 6.65(1 \mathrm{H}$, br d, $J=7.9 \mathrm{~Hz}), 6.56(1 \mathrm{H}$, br d, $J=7.9$ $\mathrm{Hz}), 5.58(1 \mathrm{H}, \mathrm{br} \mathrm{s}), 4.74(1 \mathrm{H}$, br s), $4.16(2 \mathrm{H}, \mathrm{m}), 2.27(1 \mathrm{H}, \mathrm{m}), 2.14(1 \mathrm{H}, \mathrm{m}), 1.16(3 \mathrm{H}, \mathrm{t}$, $J=7.0 \mathrm{~Hz}), 1.13(3 \mathrm{H}, \mathrm{t}, J=7.3 \mathrm{~Hz}) .{ }^{13} \mathrm{C}$ NMR $\left(\mathrm{CDCl}_{3}\right) \delta 170.7,164.0,144.4,141.6,121.1$, 120.3, 111.4, 108.8, 66.0, 62.8, 28.0, 13.7, 7.9. MS $\left(\mathrm{EI}^{+}\right): \mathrm{m} / \mathrm{z} 265\left(\mathrm{M}^{+}, 26\right), 192$ (100). Anal. Calcd for $\mathrm{C}_{13} \mathrm{H}_{15} \mathrm{NO}_{5}$ : C, 58.86; H, 5.70; N, 5.28. Found: C, 58.56; H, 5.60; N, 5.26. 27b: A colorless oil. IR $\left(\mathrm{CHCl}_{3}\right)$ 1774, $1740 \mathrm{~cm}^{-1} .{ }^{1} \mathrm{H}$ NMR $\left(\mathrm{CDCl}_{3}\right) \delta 7.09(1 \mathrm{H}, \mathrm{t}, J=8.2$ $\mathrm{Hz}), 6.77(1 \mathrm{H}, \mathrm{d}, J=8.2 \mathrm{~Hz}), 6.67(1 \mathrm{H}, \mathrm{d}, J=8.2 \mathrm{~Hz}), 5.90(1 \mathrm{H}, \mathrm{br} \mathrm{s}), 4.69(1 \mathrm{H}, \mathrm{s}), 4.10(2 \mathrm{H}$, q, $J=7.0 \mathrm{~Hz}), 3.41(1 \mathrm{H}, \mathrm{m}), 1.30(3 \mathrm{H}, \mathrm{d}, J=6.7 \mathrm{~Hz}), 1.10(3 \mathrm{H}, \mathrm{t}, J=7.0 \mathrm{~Hz}), 1.09(3 \mathrm{H}, \mathrm{d}$, $J=6.1 \mathrm{~Hz}) .{ }^{13} \mathrm{C} \mathrm{NMR}\left(\mathrm{CDCl}_{3}\right) \delta 166.1,163.9,151.5,147.6,126.9,119.0,112.0,108.6,62.7$, 59.6, 54.3, 20.5, 19.0, 13.6. $\mathrm{MS}\left(\mathrm{EI}^{+}\right): \mathrm{m} / \mathrm{z} 279\left(\mathrm{M}^{+}, 28\right), 164$ (100). HRMS $\left(\mathrm{EI}^{+}\right)$: calcd for 
$\mathrm{C}_{14} \mathrm{H}_{17} \mathrm{NO}_{5}\left(\mathrm{M}^{+}\right)$: 279.1107, Found: 279.1111.

27d: A colorless oil. IR $\left(\mathrm{CHCl}_{3}\right) 1776,1736 \mathrm{~cm}^{-1} .{ }^{1} \mathrm{H} \mathrm{NMR}\left(\mathrm{CDCl}_{3}\right) \delta 6.68(1 \mathrm{H}, \mathrm{br} \mathrm{t}, J=8.2$ $\mathrm{Hz}), 6.60(1 \mathrm{H}$, br d, $J=8.2 \mathrm{~Hz}), 6.53(1 \mathrm{H}$, br d, $J=8.2 \mathrm{~Hz}), 5.69$ (1H, br s), 5.00 (1H, br s), $4.14(2 \mathrm{H}, \mathrm{m}), 1.32(9 \mathrm{H}, \mathrm{s}), 1.13(3 \mathrm{H}, \mathrm{t}, J=7.3 \mathrm{~Hz}) .{ }^{13} \mathrm{C} \mathrm{NMR}\left(\mathrm{CDCl}_{3}\right) \delta 170.1,163.1,144.4$,

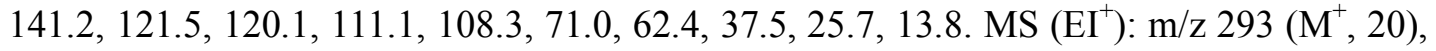
191 (100). HRMS (EI $\left.{ }^{+}\right)$: calcd for $\mathrm{C}_{15} \mathrm{H}_{19} \mathrm{NO}_{5}\left(\mathrm{M}^{+}\right)$: 293.1263, Found: 293.1271.

28a: A colorless crystal. mp 130-133 ${ }^{\circ} \mathrm{C}$ (AcOEt/hexane). IR $\left(\mathrm{CHCl}_{3}\right) 1760 \mathrm{~cm}^{-1} .{ }^{1} \mathrm{H} \mathrm{NMR}$ $\left(\mathrm{CDCl}_{3}\right) \delta 7.36(2 \mathrm{H}, \mathrm{d}, J=7.6 \mathrm{~Hz}), 7.31-7.20(3 \mathrm{H}, \mathrm{m}), 6.99(1 \mathrm{H}, \mathrm{t}, J=7.6 \mathrm{~Hz}), 6.89(1 \mathrm{H}, \mathrm{d}$, $J=7.6 \mathrm{~Hz}), 6.86(1 \mathrm{H}, \mathrm{d}, J=7.6 \mathrm{~Hz}), 6.75(1 \mathrm{H}, \mathrm{t}, J=7.6 \mathrm{~Hz}), 4.41(1 \mathrm{H}, \mathrm{br} \mathrm{s}), 2.41(1 \mathrm{H}, \mathrm{m})$, $2.04(1 \mathrm{H}, \mathrm{m}), 1.00(3 \mathrm{H}, \mathrm{t}, J=7.3 \mathrm{~Hz}) .{ }^{13} \mathrm{C} \mathrm{NMR}\left(\mathrm{CDCl}_{3}\right) \delta 166.4,140.8,139.3,132.1,128.7$, 128.0, 125.6, 125.0, 120.0, 116.5, 115.0, 64.2, 33.3, 8.1. MS (EI $\left.{ }^{+}\right): \mathrm{m} / \mathrm{z} 253\left(\mathrm{M}^{+}, 9\right), 196$ (100). Anal. Calcd for $\mathrm{C}_{16} \mathrm{H}_{15} \mathrm{NO}_{2}: \mathrm{C}, 75.87 ; \mathrm{H}, 5.97 ; \mathrm{N}, 5.53$. Found: C, 75.65; H, 6.02; N, 5.45 .

29a: A colorless oil. IR $\left(\mathrm{CHCl}_{3}\right) 1760 \mathrm{~cm}^{-1} .{ }^{1} \mathrm{H}$ NMR $\left(\mathrm{CDCl}_{3}\right) \delta$ 7.30-7.19 (5H, m), 6.64 $(1 \mathrm{H}, \mathrm{t}, J=7.9 \mathrm{~Hz}), 6.56(1 \mathrm{H}, \mathrm{d}, J=7.9 \mathrm{~Hz}), 6.50(1 \mathrm{H}, \mathrm{d}, J=7.9 \mathrm{~Hz}), 5.22(1 \mathrm{H}, \mathrm{br} \mathrm{s}), 4.51(1 \mathrm{H}$, br s), $2.40(1 \mathrm{H}, \mathrm{m}), 2.08(1 \mathrm{H}, \mathrm{m}), 1.01(3 \mathrm{H}, \mathrm{t}, J=7.3 \mathrm{~Hz}) .{ }^{13} \mathrm{C} \mathrm{NMR}\left(\mathrm{CDCl}_{3}\right) \delta 167.3,143.9$, $142.0,138.8,128.8,128.1,125.3,120.9,119.8,110.9,108.9,64.4,33.4,8.1 . \mathrm{MS}\left(\mathrm{EI}^{+}\right): \mathrm{m} / \mathrm{z}$ $269\left(\mathrm{M}^{+}, 19\right), 240(100)$. HRMS $\left(\mathrm{EI}^{+}\right)$: calcd for $\mathrm{C}_{16} \mathrm{H}_{15} \mathrm{NO}_{3}\left(\mathrm{M}^{+}\right)$: 269.1052, Found: 269.1057.

29b: A colorless crystal. mp 158-160 ${ }^{\circ} \mathrm{C}$ (AcOEt/hexane). IR $\left(\mathrm{CHCl}_{3}\right) 1756 \mathrm{~cm}^{-1} .{ }^{1} \mathrm{H}$ NMR $\left(\mathrm{CDCl}_{3}\right) \delta 7.38-7.18(5 \mathrm{H}, \mathrm{m}), 6.62(1 \mathrm{H}$, br t,$J=7.9 \mathrm{~Hz}), 6.57(1 \mathrm{H}, \mathrm{br} \mathrm{d}, J=7.9 \mathrm{~Hz}), 6.48(1 \mathrm{H}$, br d, $J=7.9 \mathrm{~Hz}), 5.23(1 \mathrm{H}$, br s), $4.49(1 \mathrm{H}$, br s), $2.87(1 \mathrm{H}, \mathrm{m}), 1.25(3 \mathrm{H}, \mathrm{d}, J=6.7 \mathrm{~Hz}), 0.71$ $(3 \mathrm{H}, \mathrm{d}, J=7.0 \mathrm{~Hz}) .{ }^{13} \mathrm{C} \mathrm{NMR}\left(\mathrm{CDCl}_{3}\right) \delta 167.4,143.4,141.2,138.4,128.8,128.0,125.7$, 121.1, 119.2, 110.9, 109.0, 67.4, 36.7, 18.3, 15.5. $\mathrm{MS}\left(\mathrm{EI}^{+}\right): \mathrm{m} / \mathrm{z} 283\left(\mathrm{M}^{+}, 7\right), 240(100)$. Anal. Calcd for $\mathrm{C}_{17} \mathrm{H}_{17} \mathrm{NO}_{3}$ : C, 72.07; H, 6.05; N, 4.94. Found: C, 72.00; H, 5.98; N, 4.93.

29d: A colorless oil. IR $\left(\mathrm{CHCl}_{3}\right)$ 1761, $1733 \mathrm{~cm}^{-1} .{ }^{1} \mathrm{H} \mathrm{NMR}\left(\mathrm{CDCl}_{3}\right) \delta$ 7.33-7.16 (5H, m), $6.56(1 \mathrm{H}$, br t, $J=7.9 \mathrm{~Hz}), 6.50(1 \mathrm{H}$, br d, $J=7.9 \mathrm{~Hz}), 6.38(1 \mathrm{H}$, br d, $J=7.9 \mathrm{~Hz}), 5.55(1 \mathrm{H}, \mathrm{br}$ s), $4.88\left(1 \mathrm{H}\right.$, br s), $1.22(9 \mathrm{H}, \mathrm{s}) .{ }^{13} \mathrm{C} \mathrm{NMR}\left(\mathrm{CDCl}_{3}\right) \delta 165.5,144.0,142.3,134.8,128.0$, 127.8, 127.5, 121.3, 119.8, 110.4, 108.5, 69.5, 38.1, 26.0. MS (EI $\left.{ }^{+}\right): \mathrm{m} / \mathrm{z} 297\left(\mathrm{M}^{+}, 3\right), 240$ (100). HRMS (EI ${ }^{+}$): calcd for $\mathrm{C}_{18} \mathrm{H}_{19} \mathrm{NO}_{3}\left(\mathrm{M}^{+}\right)$: 297.1365, Found: 297.1350 . 
General Procedure for Aminolysis of Cyclic Esters 30 and 35. To a solution of $\mathbf{3 0}$ or $35^{11}(0.36 \mathrm{mmol})$ in THF $(10 \mathrm{~mL})$ were added $\mathrm{PhCH}_{2} \mathrm{CH}_{2} \mathrm{NH}_{2}(1.1 \mathrm{~g}, 9.0 \mathrm{mmol})$ and 2-pyridinol (170 mg, $1.8 \mathrm{mmol})$ under nitrogen atmosphere at $20^{\circ} \mathrm{C}$. After being stirred at the same temperature for $12 \mathrm{~h}$, the reaction mixture was diluted with water and then extracted with AcOEt. The organic phase was dried over $\mathrm{MgSO}_{4}$ and concentrated at reduced pressure. Purification of the residue by column chromatography (hexane:AcOEt=4:1) afforded $\mathbf{3 1}$ or $\mathbf{3 6}$.

31: A colorless oil. IR $\left(\mathrm{CHCl}_{3}\right) 3440,3310,1636 \mathrm{~cm}^{-1} .{ }^{1} \mathrm{H} \mathrm{NMR}\left(\mathrm{CDCl}_{3}\right) \delta 7.96(2 \mathrm{H}, \mathrm{s})$, 7.31-7.20 (3H, m), 7.12 (2H, d, J=7.0 Hz), 6.30 (2H, s), 5.91 (1H, br m), 3.60 (2H, m), 3.32 $(1 \mathrm{H}, \mathrm{br} \mathrm{s}), 2.83(2 \mathrm{H}, \mathrm{t}, J=6.7 \mathrm{~Hz}), 2.50(2 \mathrm{H}, \mathrm{q}, J=7.6 \mathrm{~Hz}), 1.78-1.64(2 \mathrm{H}, \mathrm{m}), 1.18(3 \mathrm{H}, \mathrm{t}$, $J=7.6 \mathrm{~Hz}), 1.09(3 \mathrm{H}, \mathrm{s}), 0.93(3 \mathrm{H}, \mathrm{t}, J=7.6 \mathrm{~Hz}) .{ }^{13} \mathrm{C} \mathrm{NMR}\left(\mathrm{CDCl}_{3}\right) \delta 180.5,155.2,144.6$, 138.2, 128.8, 128.7, 126.8, 113.8, 106.0, 63.0, 41.5, 36.4, 35.2, 28.7, 21.0, 15.1, 8.2. MS $\left(\mathrm{FAB}^{+}\right): \mathrm{m} / \mathrm{z} 357\left(\mathrm{M}+\mathrm{H}^{+}, 47\right), 208(100)$. HRMS $\left(\mathrm{FAB}^{+}\right)$: calcd for $\mathrm{C}_{21} \mathrm{H}_{29} \mathrm{~N}_{2} \mathrm{O}_{3}\left(\mathrm{M}+\mathrm{H}^{+}\right)$: 357.2178, Found: 357.2169. $[\alpha]^{24}+14.9\left(c 1.0, \mathrm{CHCl}_{3}\right)$.

36: A colorless solid. IR $\left(\mathrm{CHCl}_{3}\right) 3350,1656 \mathrm{~cm}^{-1} .{ }^{1} \mathrm{H}$ NMR $\left(\mathrm{CDCl}_{3}\right) \delta$ 7.34-7.13 (10H, m), $3.76(1 \mathrm{H}, \mathrm{dd}, J=8.6,4.6 \mathrm{~Hz}), 3.57(1 \mathrm{H}, \mathrm{dd}, J=10.7,4.6 \mathrm{~Hz}), 3.47-3.36(2 \mathrm{H}, \mathrm{m}), 3.28(1 \mathrm{H}$, m), $2.66(2 \mathrm{H}, \mathrm{t}, J=7.0 \mathrm{~Hz}), 2.10(3 \mathrm{H}$, br s), $1.70(1 \mathrm{H}, \mathrm{m}), 1.63(1 \mathrm{H}, \mathrm{m}), 1.07(3 \mathrm{H}, \mathrm{s}), 0.76$ $(3 \mathrm{H}, \mathrm{t}, J=7.6 \mathrm{~Hz}) .{ }^{13} \mathrm{C} \mathrm{NMR}\left(\mathrm{CDCl}_{3}\right) \delta 176.5,142.8,139.2,128.8,128.6(2 \mathrm{C}), 127.4,127.1$, 126.4, 67.7, 62.7, 59.2, 40.1, 35.4, 30.6, 23.8, 8.0. MS (FAB $\left.{ }^{+}\right): \mathrm{m} / \mathrm{z} 341\left(\mathrm{M}+\mathrm{H}^{+}, 94\right), 192$ (100). HRMS (FAB ${ }^{+}$): calcd for $\mathrm{C}_{21} \mathrm{H}_{29} \mathrm{~N}_{2} \mathrm{O}_{2}\left(\mathrm{M}+\mathrm{H}^{+}\right)$: 341.2229, Found: 341.2232. Anal. Calcd for $\mathrm{C}_{21} \mathrm{H}_{28} \mathrm{~N}_{2} \mathrm{O}_{2}$ : C, 74.08; H, 8.29; N, 8.23. Found: C, 73.82; H, 8.27; N, 8.15. $[\alpha]^{26}{ }_{D}$ -28.7 (c 1.0, $\left.\mathrm{CHCl}_{3}\right)$.

Methylation of 31. To a solution of $31(249 \mathrm{mg}, 0.70 \mathrm{mmol})$ in DMF $(15 \mathrm{~mL})$ were added $\mathrm{K}_{2} \mathrm{CO}_{3}(496 \mathrm{mg}, 3.4 \mathrm{mmol})$ and $\mathrm{MeI}(0.24 \mathrm{~mL}, 3.4 \mathrm{mmol})$ under nitrogen atmosphere at $20{ }^{\circ} \mathrm{C}$. After being stirred at the same temperature for $5 \mathrm{~h}$, the reaction mixture was diluted with $\mathrm{Et}_{2} \mathrm{O}$ and then washed with water. The organic phase was dried over $\mathrm{MgSO}_{4}$ and concentrated at reduced pressure. Purification of the residue by column chromatography (hexane:AcOEt=6:1) afforded 32 (263 mg, 98\%).

32: A colorless oil. IR $\left(\mathrm{CHCl}_{3}\right) 3383,1652 \mathrm{~cm}^{-1} .{ }^{1} \mathrm{H} \mathrm{NMR}\left(\mathrm{CDCl}_{3}\right) \delta 7.66(1 \mathrm{H}, \mathrm{br} \mathrm{m})$, 7.28-7.17 (5H, m), $6.35(2 \mathrm{H}, \mathrm{s}), 4.30(1 \mathrm{H}, \mathrm{br} \mathrm{s}), 3.75(6 \mathrm{H}, \mathrm{s}), 3.58(1 \mathrm{H}, \mathrm{m}), 3.47(1 \mathrm{H}, \mathrm{m})$, 
$2.82(2 \mathrm{H}, \mathrm{m}), 2.58(2 \mathrm{H}, \mathrm{q}, J=7.6 \mathrm{~Hz}), 1.81-1.69(2 \mathrm{H}, \mathrm{m}), 1.22(3 \mathrm{H}, \mathrm{t}, J=7.6 \mathrm{~Hz}), 1.16(3 \mathrm{H}$, s), $0.88(3 \mathrm{H}, \mathrm{t}, J=7.6 \mathrm{~Hz}) .{ }^{13} \mathrm{C} \mathrm{NMR}\left(\mathrm{CDCl}_{3}\right) \delta 178.3,151.3,139.6,137.4,128.8,128.4$, 126.2, 121.5, 103.7, 61.9, 55.4, 40.7, 36.2, 33.5, 29.0, 21.3, 15.6, 8.1. MS (EI $\left.{ }^{+}\right): \mathrm{m} / \mathrm{z} 384$ $\left(\mathrm{M}^{+}, 4\right), 236(100)$. HRMS $\left(\mathrm{EI}^{+}\right)$: calcd for $\mathrm{C}_{23} \mathrm{H}_{32} \mathrm{~N}_{2} \mathrm{O}_{3}\left(\mathrm{M}^{+}\right)$: 384.2413, Found: 384.2404 . $[\alpha]^{25}+30.1\left(c 0.9, \mathrm{CHCl}_{3}\right)$.

Reaction of 32 with CAN. To a solution of $32(22.7 \mathrm{mg}, 0.06 \mathrm{mmol})$ in wet $\mathrm{MeOH}(1 \mathrm{~mL})$ was added a solution of cerium ammonium nitrare (132 $\mathrm{mg}, 0.24 \mathrm{mmol})$ in wet $\mathrm{MeOH}(1$ $\mathrm{mL}$ ) under nitrogen atmosphere at $-45^{\circ} \mathrm{C}$. After being stirred at the same temperature for 2 $\mathrm{h}$, the reaction mixture was diluted with water, and then solid $\mathrm{Na}_{2} \mathrm{CO}_{3}$ was added till $\mathrm{pH}$ of solution became $>7$. Insoluble inorganic materials were filtered and the aqueous layer was extracted with AcOEt. The organic phase was dried over $\mathrm{MgSO}_{4}$ and concentrated at reduced pressure. Purification of the residue by column chromatography $\left(\mathrm{CH}_{3} \mathrm{Cl}: \mathrm{MeOH}=10: 1\right)$ afforded $33(8.2 \mathrm{mg}, 62 \%)$. The amino acid derivative 33 was also prepared by hydrogenolysis of $\mathbf{3 6}$ under the standard reaction conditions.

33: A colorless oil. IR $\left(\mathrm{CHCl}_{3}\right) 3367,1654 \mathrm{~cm}^{-1} .{ }^{1} \mathrm{H} \mathrm{NMR}\left(\mathrm{CDCl}_{3}\right) \delta 7.60(1 \mathrm{H}$, br m$)$, 7.25-7.11 (5H, m), 3.48-3.36 (2H, m), $2.74(2 \mathrm{H}, \mathrm{m}), 1.77(1 \mathrm{H}, \mathrm{m}), 1.49(2 \mathrm{H}, \mathrm{br} \mathrm{s}), 1.39(1 \mathrm{H}$, m), $1.19(3 \mathrm{H}, \mathrm{s}), 0.74(3 \mathrm{H}, \mathrm{t}, J=7.6 \mathrm{~Hz}) .{ }^{13} \mathrm{C} \mathrm{NMR}\left(\mathrm{CDCl}_{3}\right) \delta 176.7,139.1,128.7,128.5$,

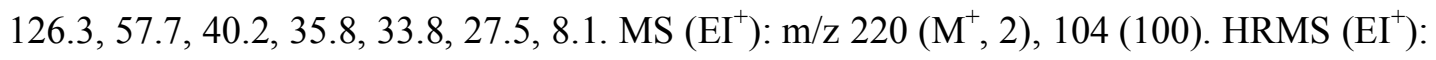
calcd for $\mathrm{C}_{13} \mathrm{H}_{20} \mathrm{~N}_{2} \mathrm{O}\left(\mathrm{M}^{+}\right)$: 220.1576, Found: 220.1578 . A sample from 32 gave $[\alpha]^{25}$ $+14.0\left(c\right.$ 0.4, $\left.\mathrm{CHCl}_{3}\right)$. A sample from 36 gave $[\alpha]^{25}{ }_{\mathrm{D}}+18.8\left(c 1.2, \mathrm{CHCl}_{3}\right)$.

\section{A hypothesis for the reversal in enantioselectivity between the zinc- and copper-mediated reactions.}
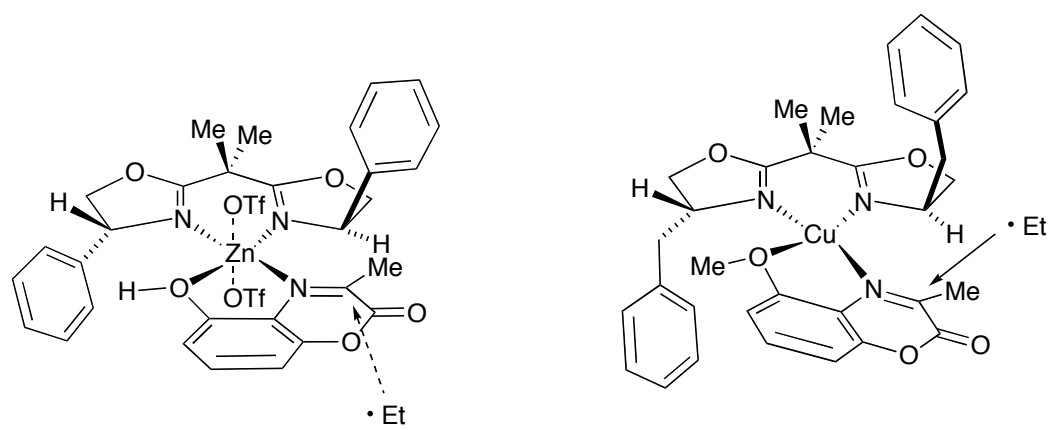


\section{References}

(1) For aldimine 4, see: (a) Gizecki, P.; Ait Youcef, R.; Poulard, C.; Dhal, R.; Dujardin, G. Tetrahedron Lett. 2004, 45, 9589. (b) Wenzel, A. G.; Jacobsen, E. N. J. Am. Chem. Soc. 2002, 124, 12964. (c) Kanazawa, A. M.; Denis, J.-N.; Greene, A. E. J. Org. Chem. 1994, 59, 1238.

(2) For aldimine 5, see: (a) Trost, B. M.; Jonasson, C. Angew. Chem., Int. Ed. 2003, 42, 2063. (b) Vidal, J.; Damestoy, S.; Guy, L.; Hannachi, J.-C.; Aubry, A.; Collet, A. Chem. Eur. J. 1997, 3, 1691. (c) Kupfer, R.; Meier, S.; Wuerthwein, E. U. Synthesis 1984, 688.

(3) For aldimine 6, see: (a) Casey, C. P.; Johnson, J. B. J. Am. Chem. Soc. 2005, 127, 1883. (b) Chakraborti, A. K.; Bhagat, S.; Rudrawar, S. Tetrahedron Lett. 2004, 45, 7641. (c) Vazquez, M. A.; Landa, M.; Reyes, L.; Miranda, R.; Tamariz, J.; Delgado, F. Synth. Commun. 2004, 34, 2705.

(4) For aldimine 7, see: (a) Lopez, C.; Caubet, A.; Perez, S.; Solans, X.; Font-Bardia, M. J. Organomet. Chem. 2003, 681, 82. (b) Alva Astudillo, M. E.; Chokotho, N. C. J.; Jarvis, T. C.; Johnson, C. D.; Lewis, C. C.; McDonnell, P. D. Tetrahedron 1985, 41, 5919.

(5) For product 8a, see: (a) Lebel, H.; Leogane, O. Org. Lett. 2005, 7, 4107. (b) Park, Y. S.; Boys, M. L.; Beak, P. J. Am. Chem. Soc. 1996, 118, 3757.

(6) For product 9a, see: (a) Arai, S.; Bellemin-Laponnaz, S.; Fu, G. C. Angew. Chem., Int. Ed. 2001, 40, 234. (b) Katritzky, A. R.; Harris, P. A. Tetrahedron Asymmetry 1992, 3, 437.

(7) For product 10a, see: (a) Denhez, C.; Vasse, J.-L.; Szymoniak, J. Synthesis 2005, 2075. (b) Cho, B. T.; Kang, S. K. Tetrahedron 2005, 61, 5725. (c) Tsvelikhovsky, D.; Gelman, D.; Molander, G. A.; Blum, J. Org. Lett. 2004, 6, 1995. (d) Alonso, E.; Ramon, D. J.; Yus, M. Tetrahedron 1996, 52, 14341.

(8) For product 11a, see: Akullian, L. C.; Porter, J. R.; Traverse, J. F.; Snapper, M. L.; Hoveyda, A. H. Adv. Synth. Catal. 2005, 347, 417.

(9) Moffett, R. B. J. Med. Chem. 1966, 9, 475.

(10) For ketimine 18, see: (a) Saitz, C.; Rodriguez, H.; Marquez, A.; Canete, A.; Jullian, C.; Zanocco, A. Synth. Commun. 2001, 31, 135. (b) Chioccara, F.; Ponsiglione, E.; Prota, 
G.; Thomson, R. H. Tetrahedron 1976, 32, 2033.

(11) (a) Miyabe, H.; Yamaoka, Y.; Takemoto, Y. J. Org. Chem. 2005, 70, 3324. (b) Harwood, L. M.; Vines, K. J.; Drew, M. G. B. Synlett 1996, 1051. 


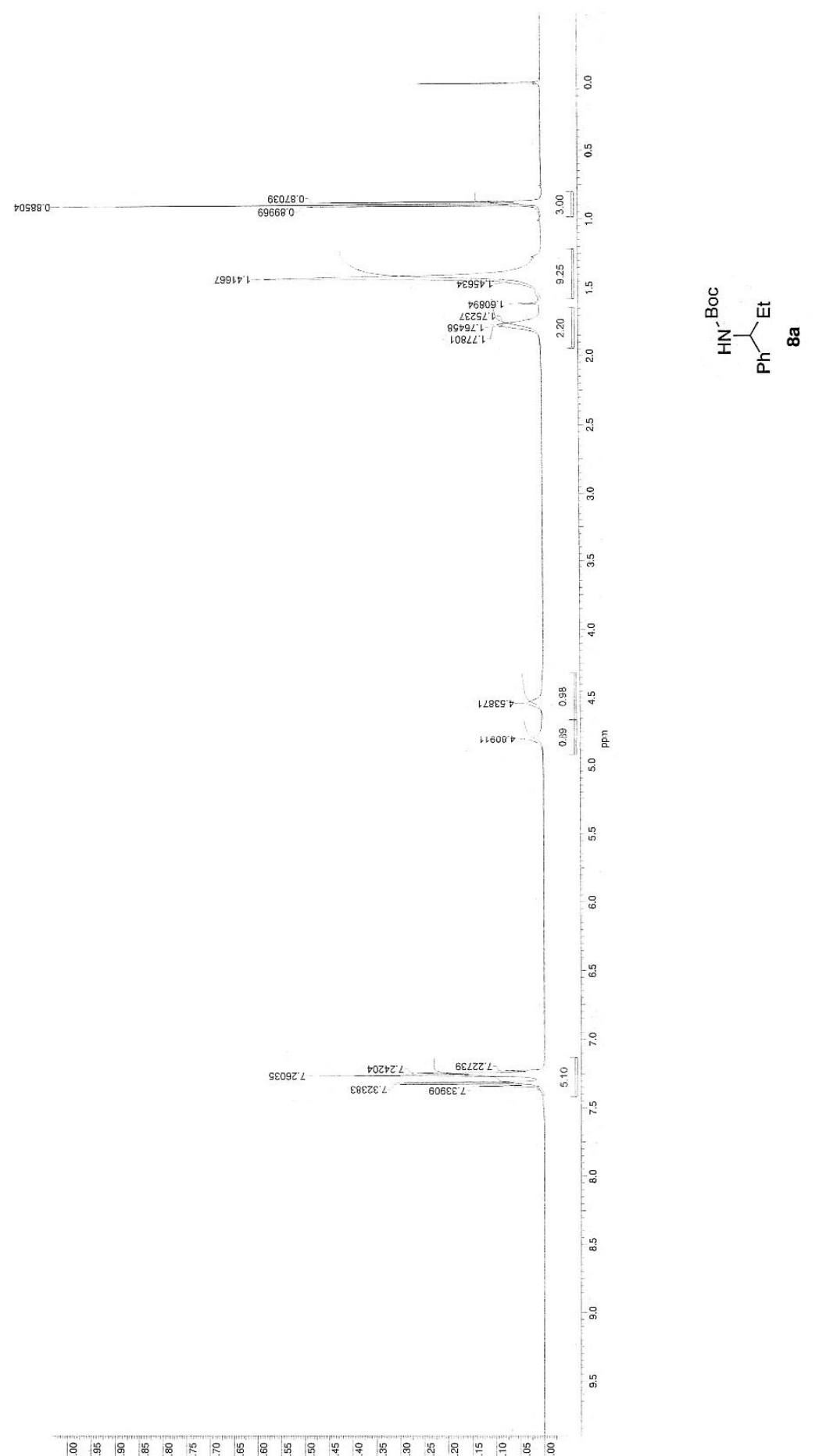

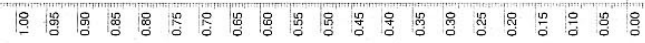

$\mathrm{S} 14$ 

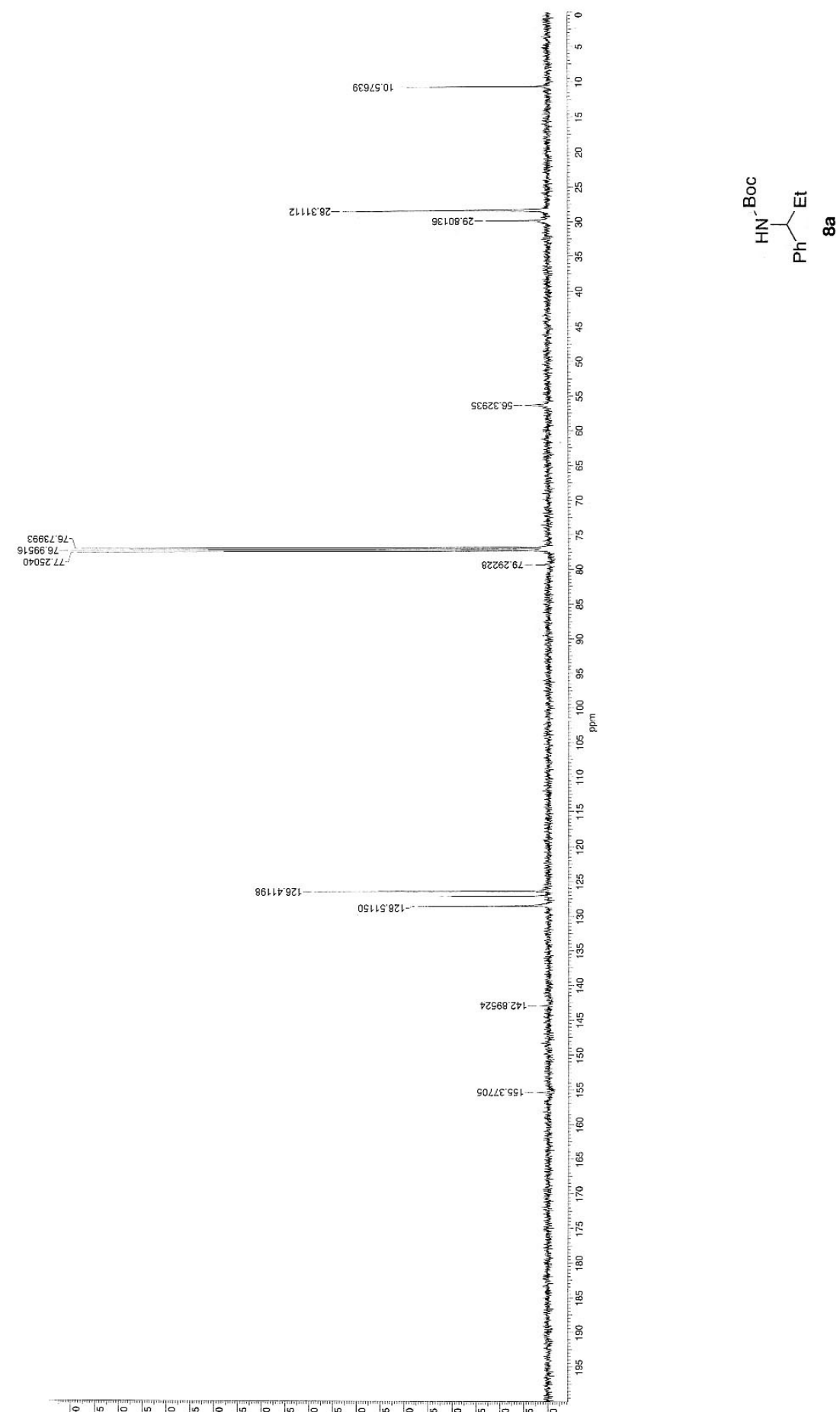

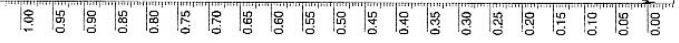

$\mathrm{S} 15$ 


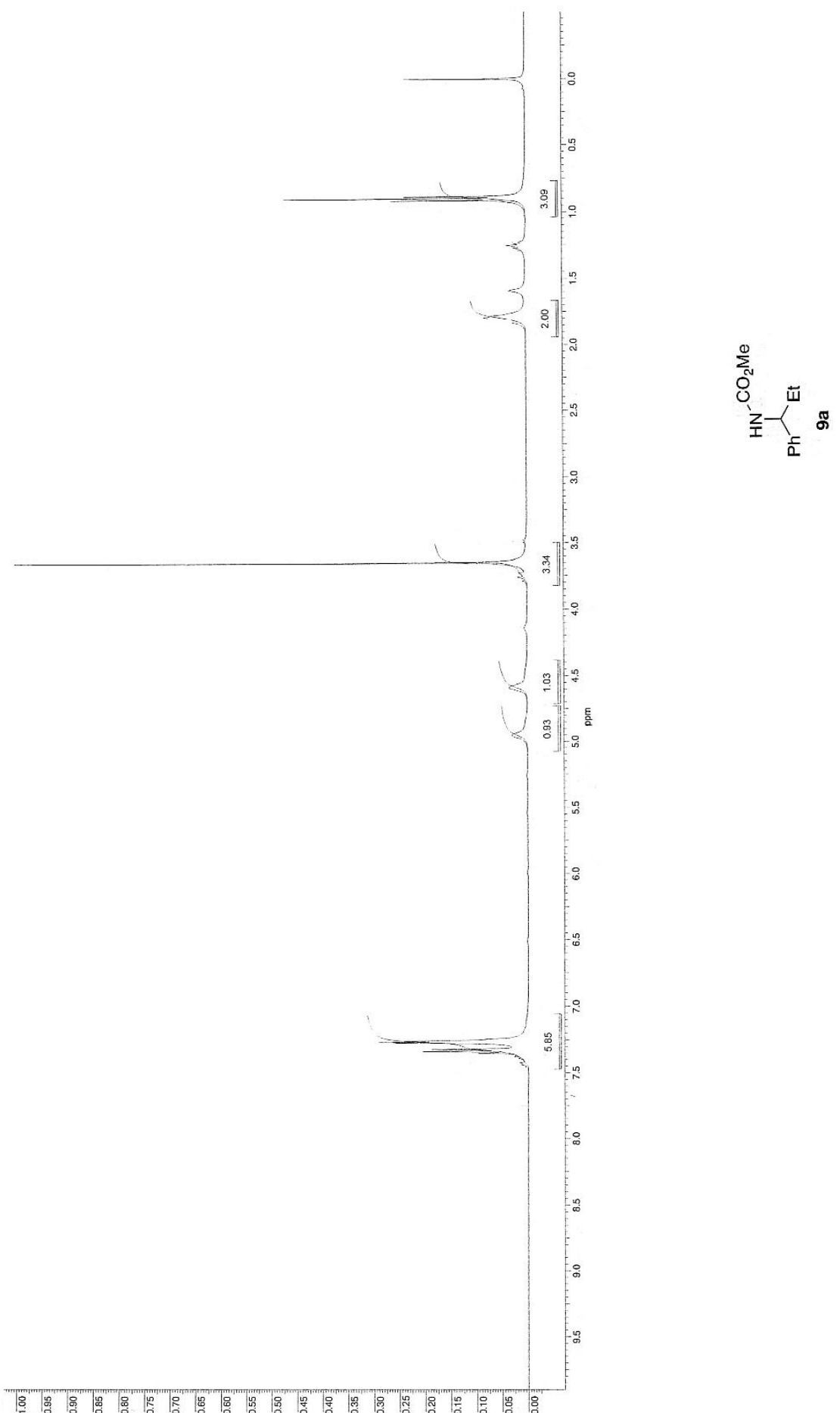

S16 

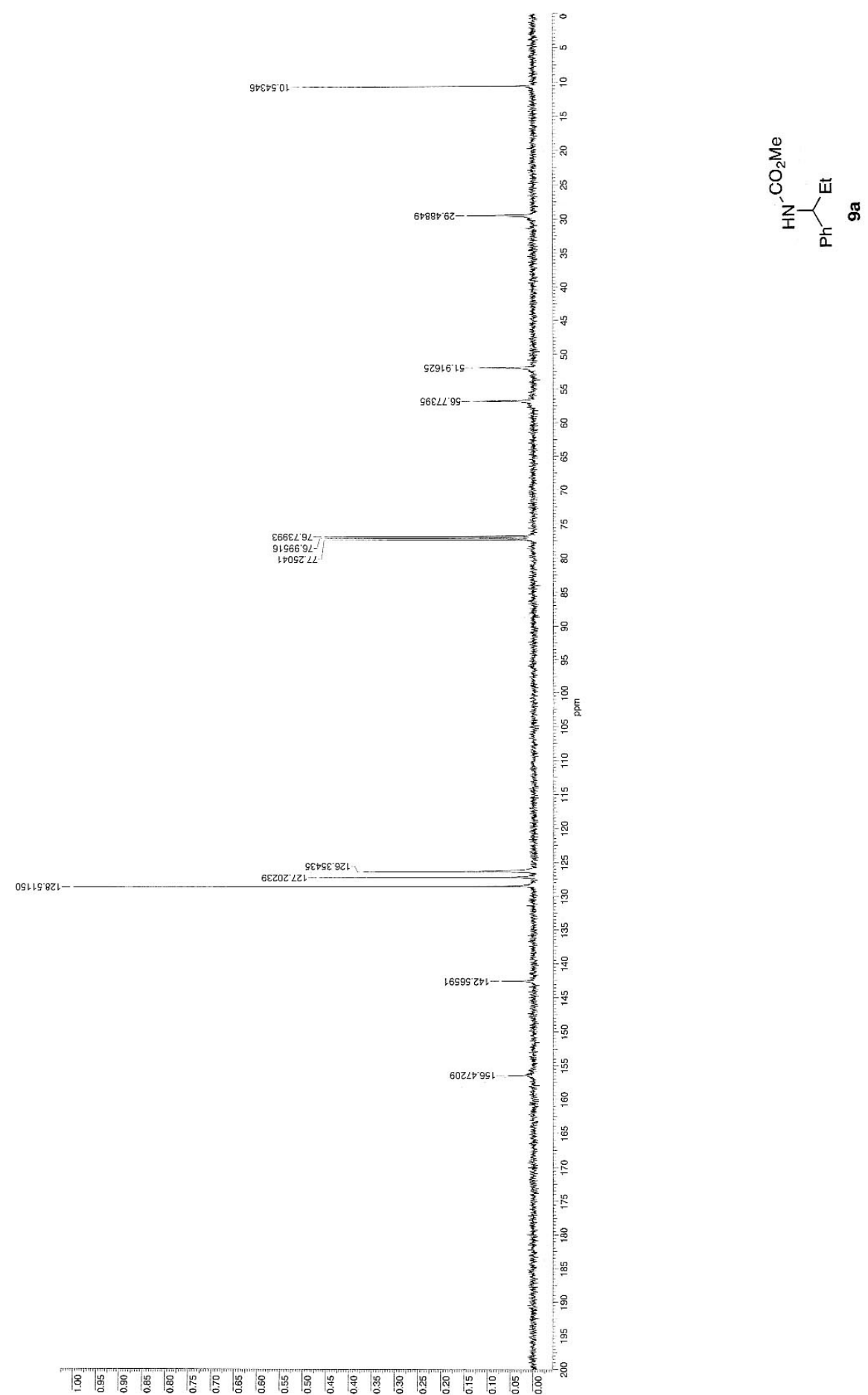


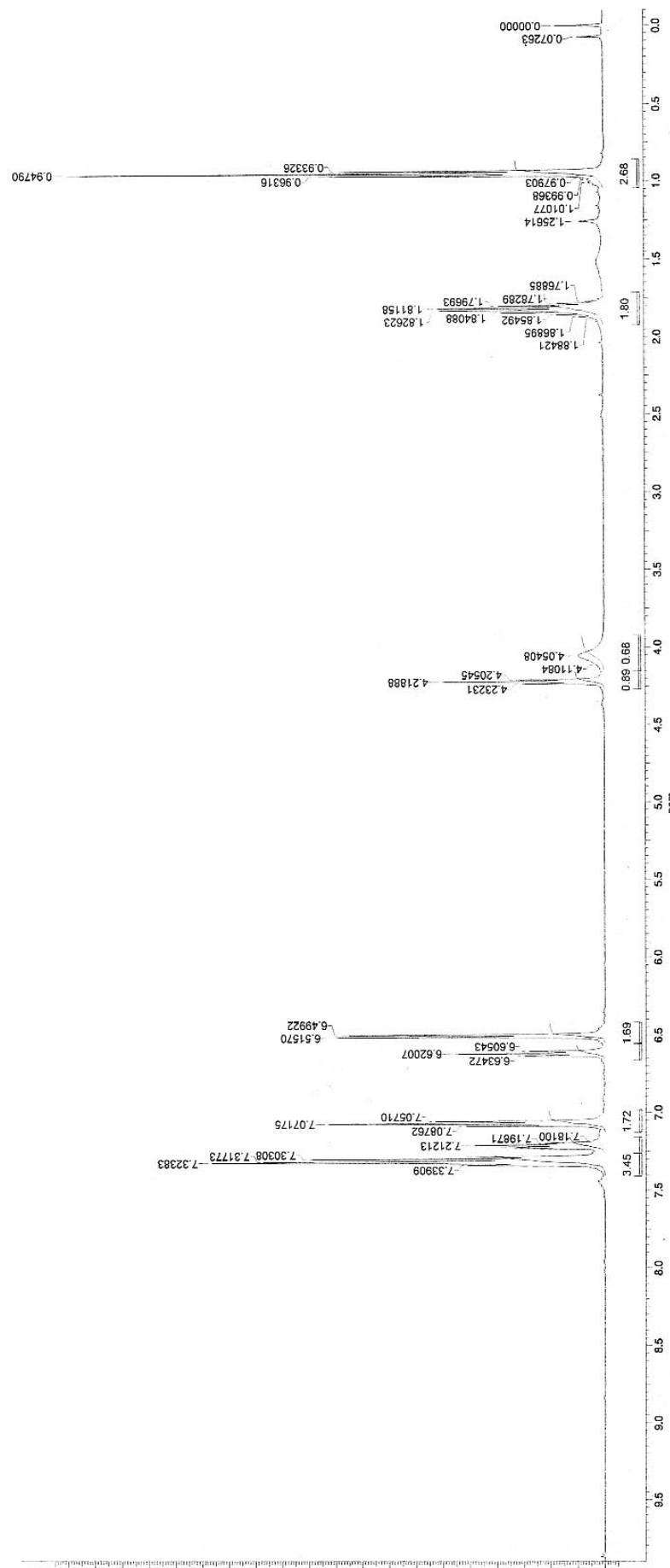

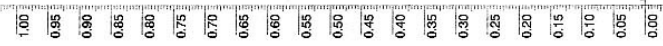

$\mathrm{S} 18$ 


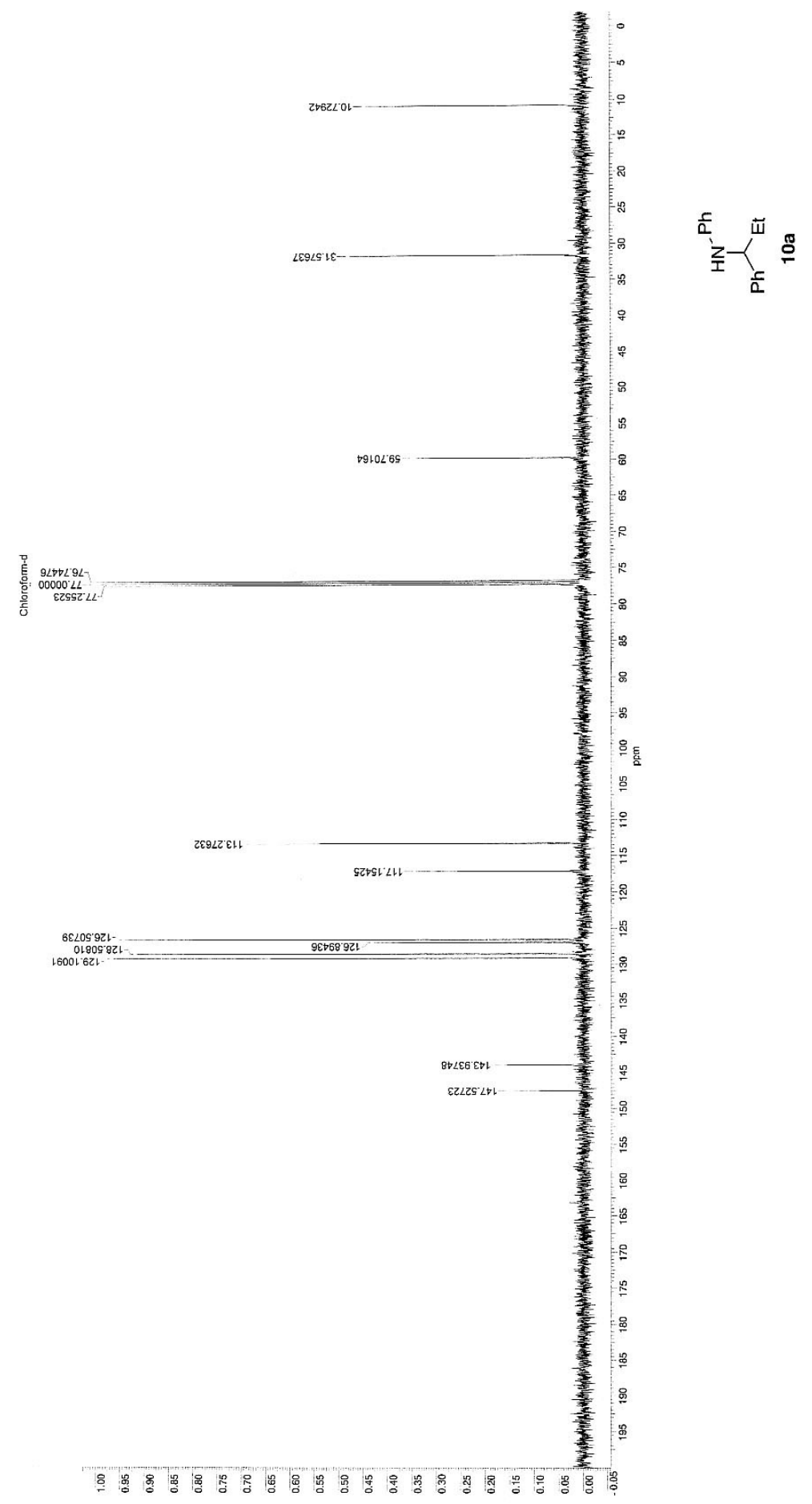

S19 


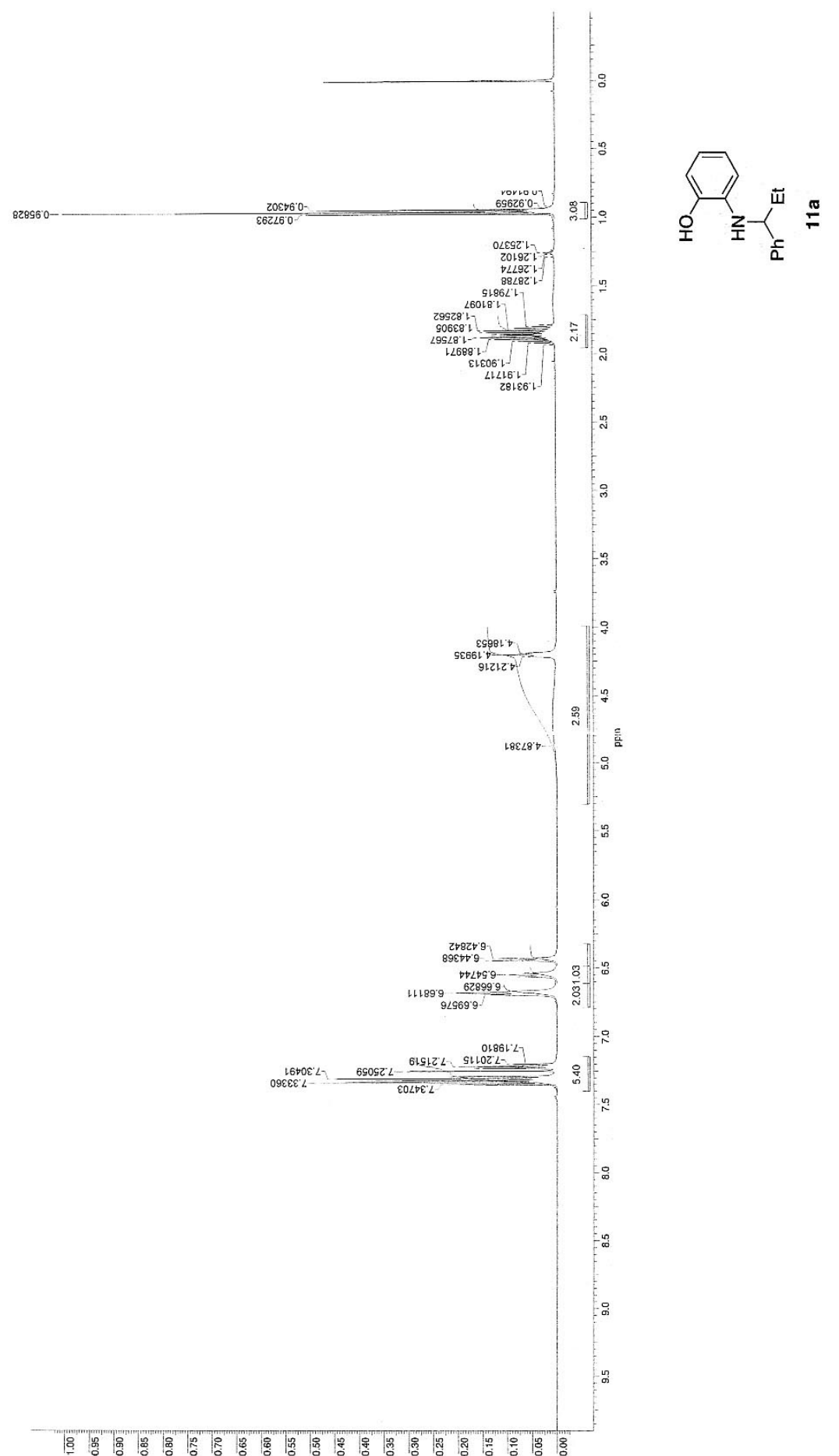




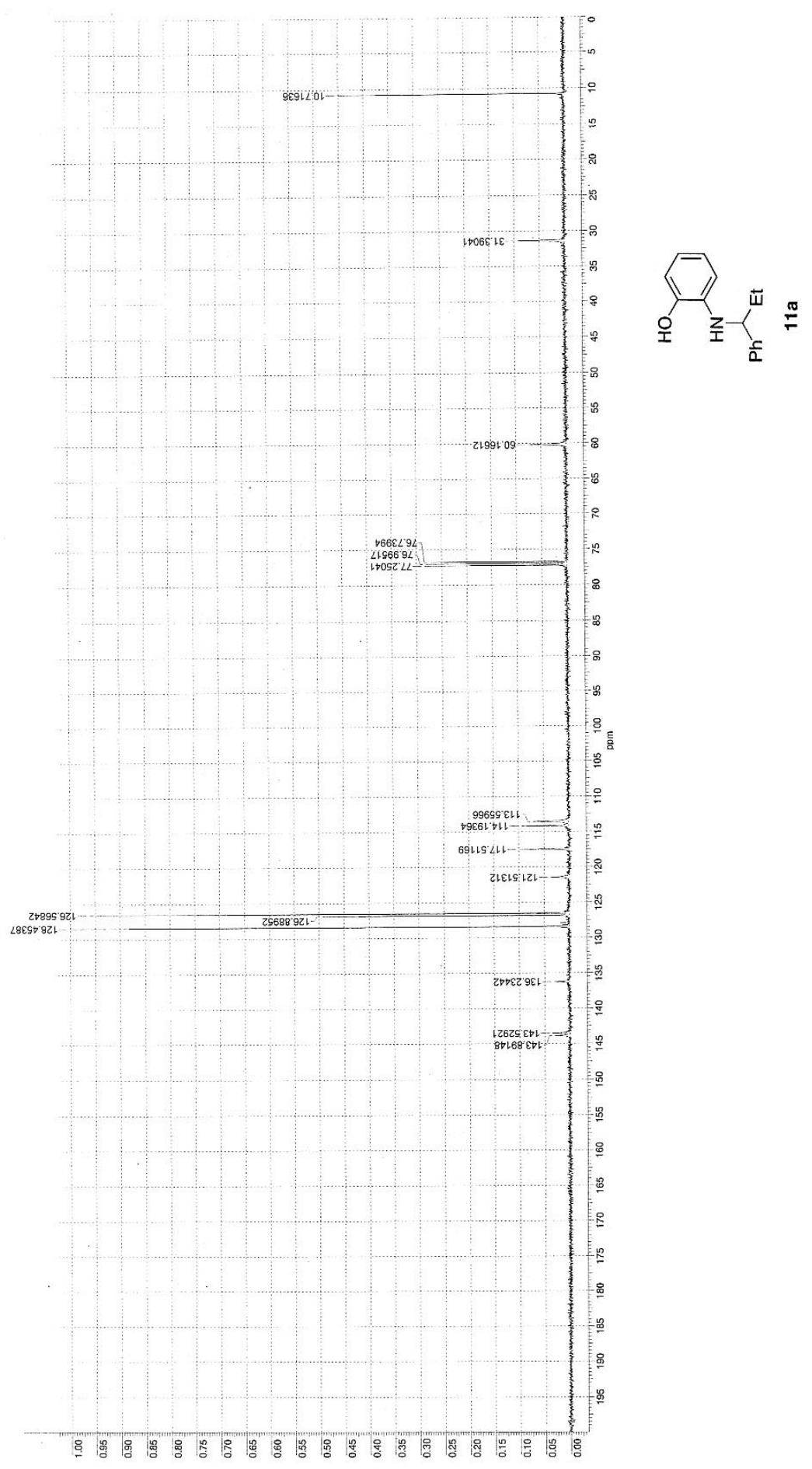

S21 


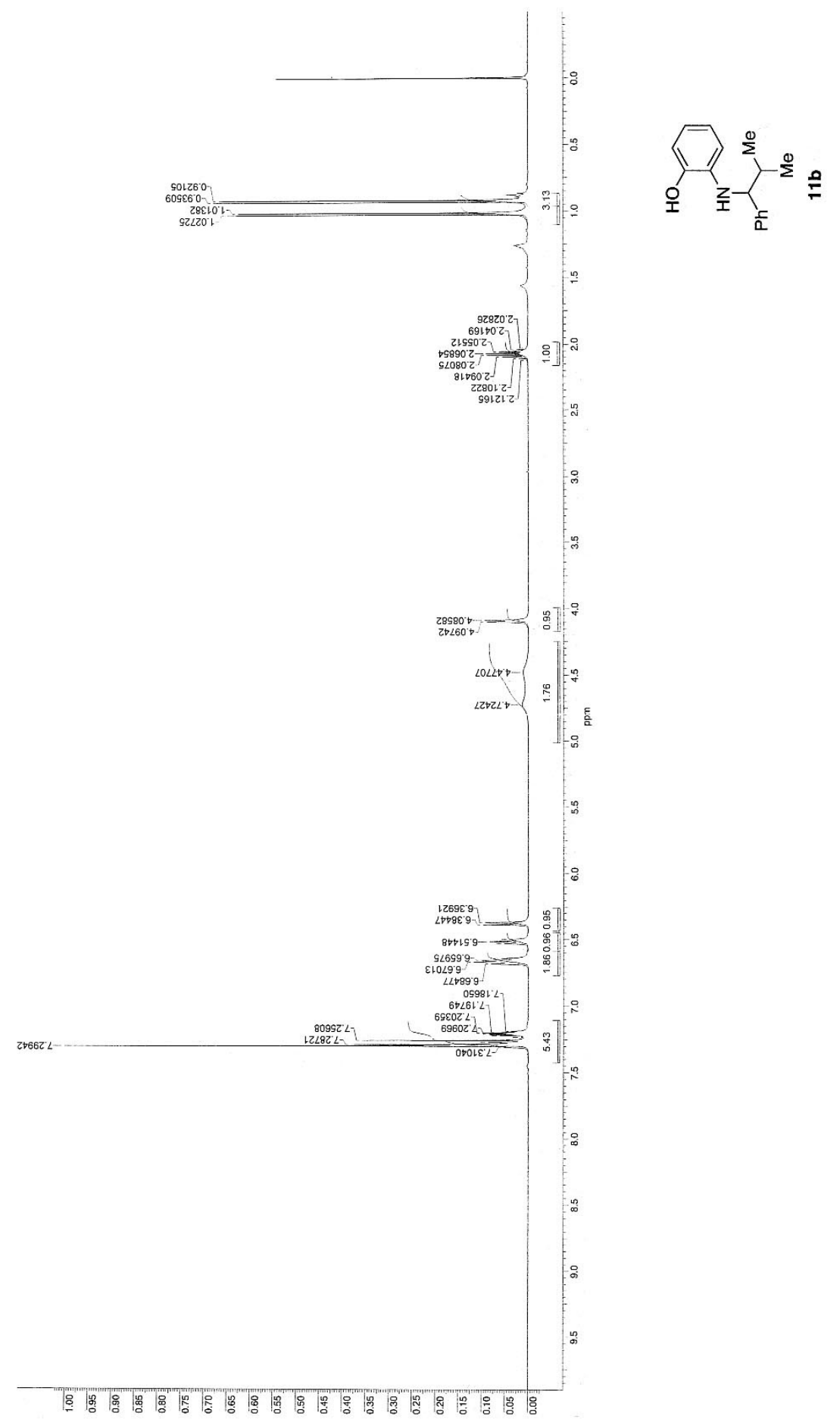




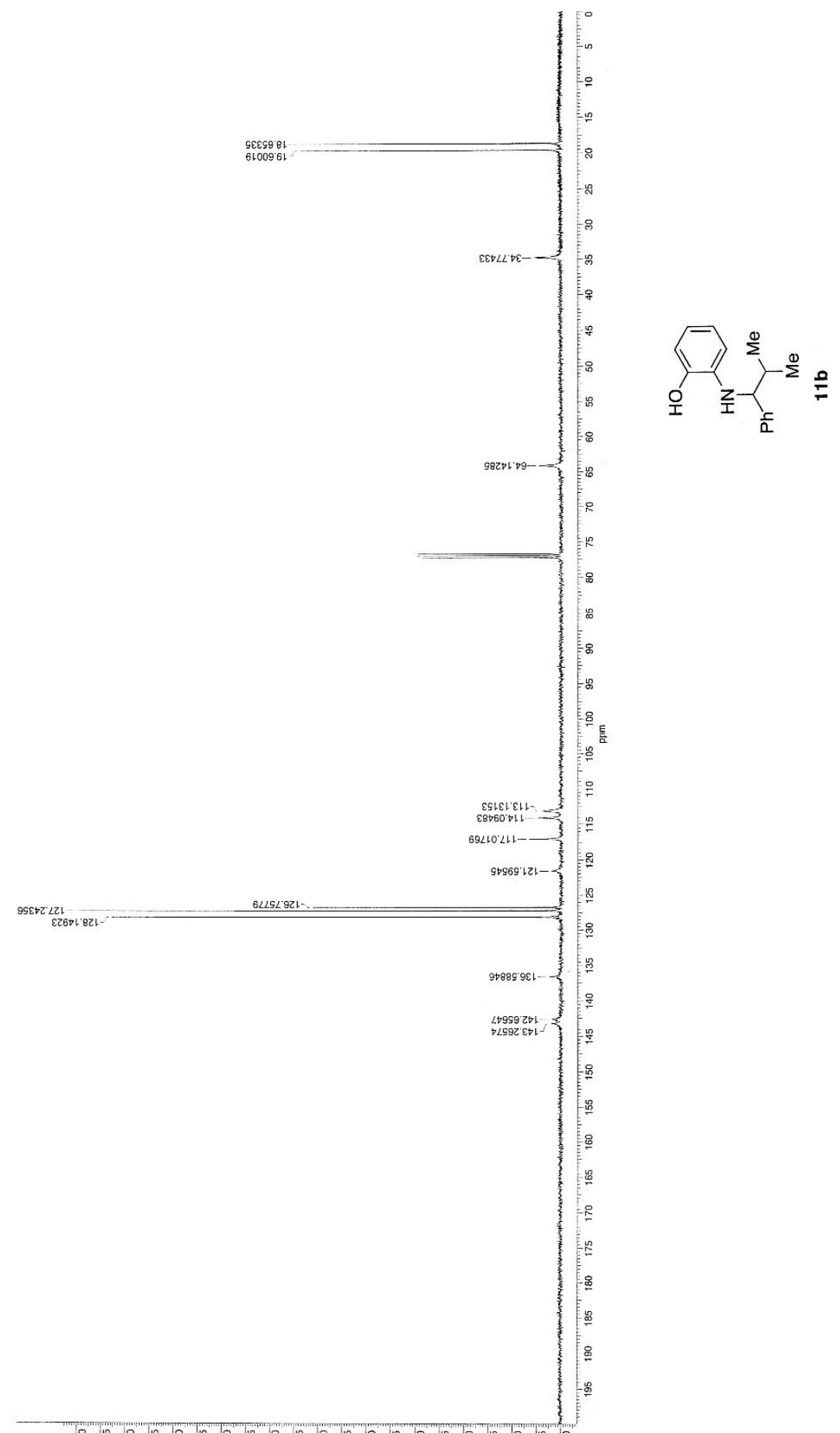

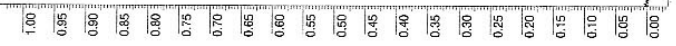




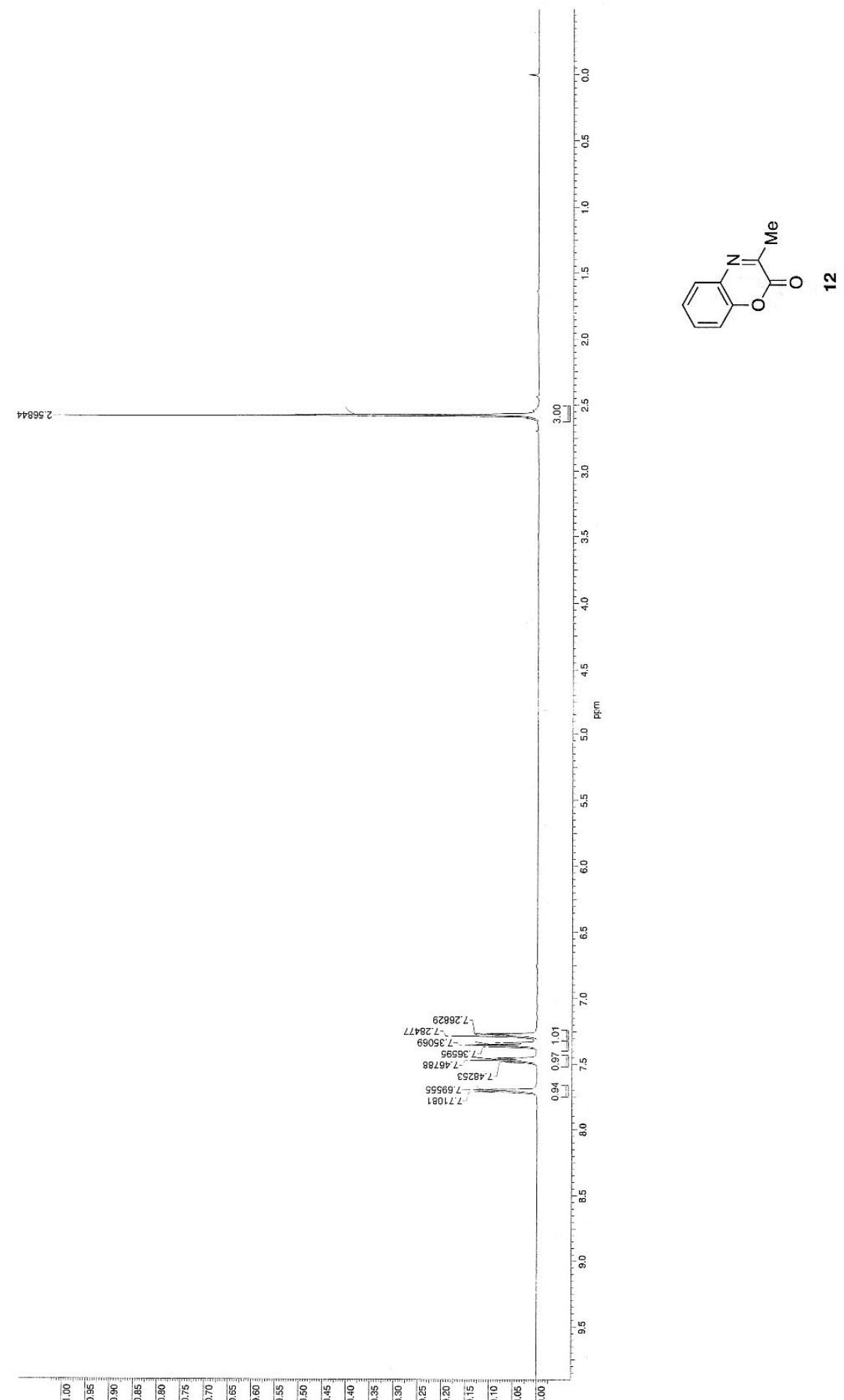

S24 


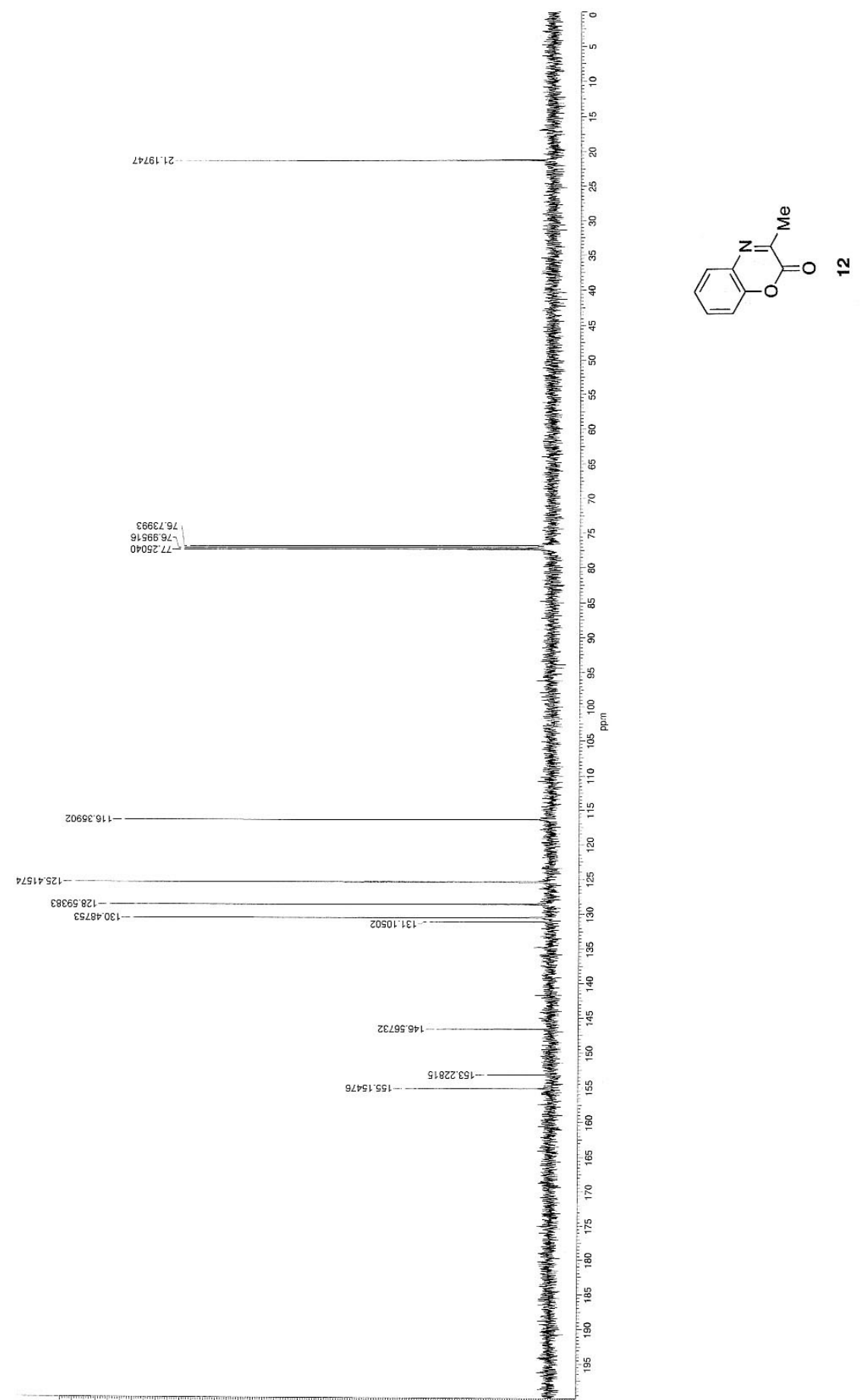

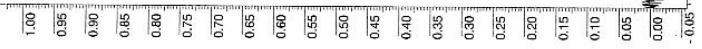




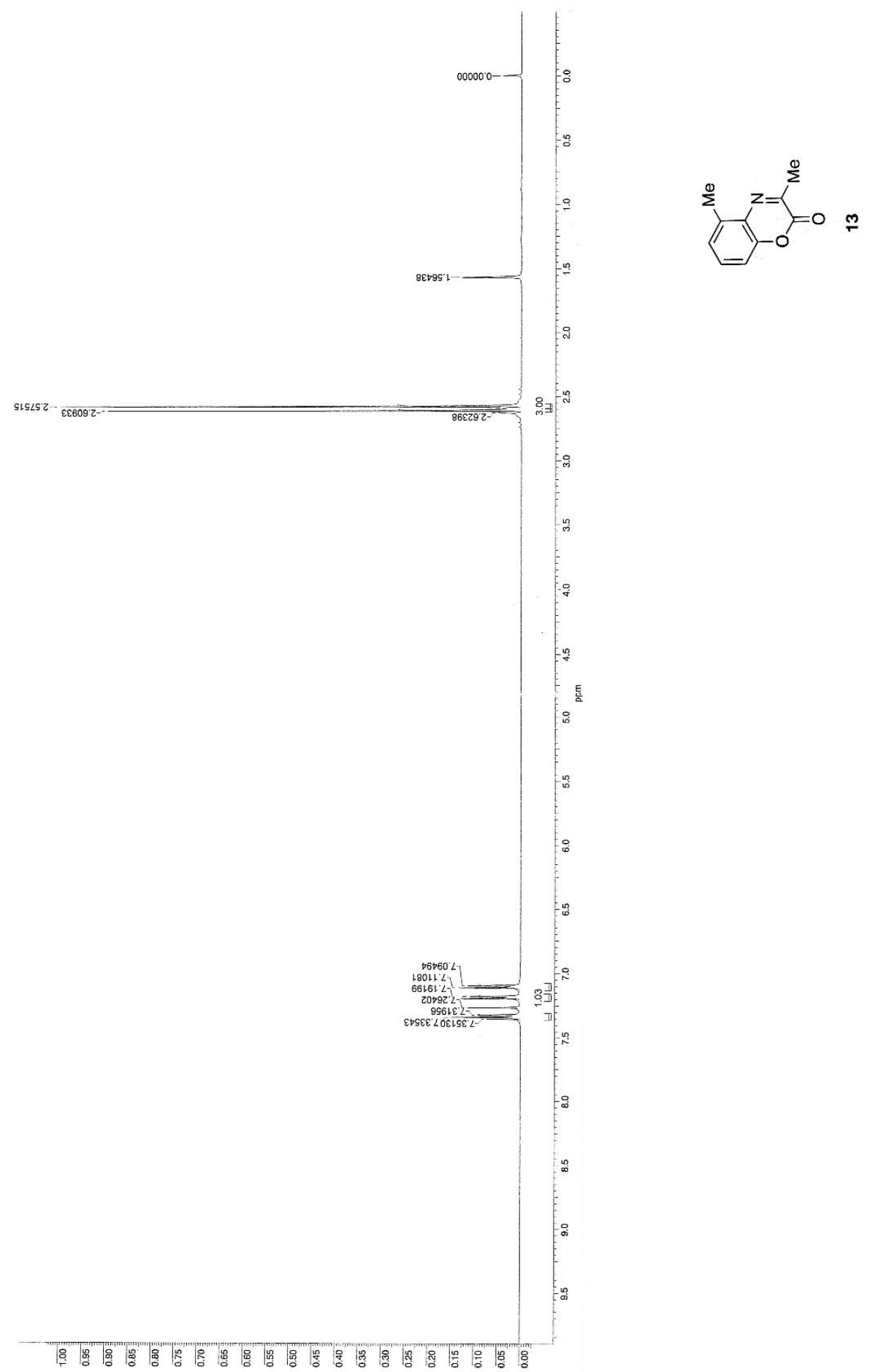

S26 


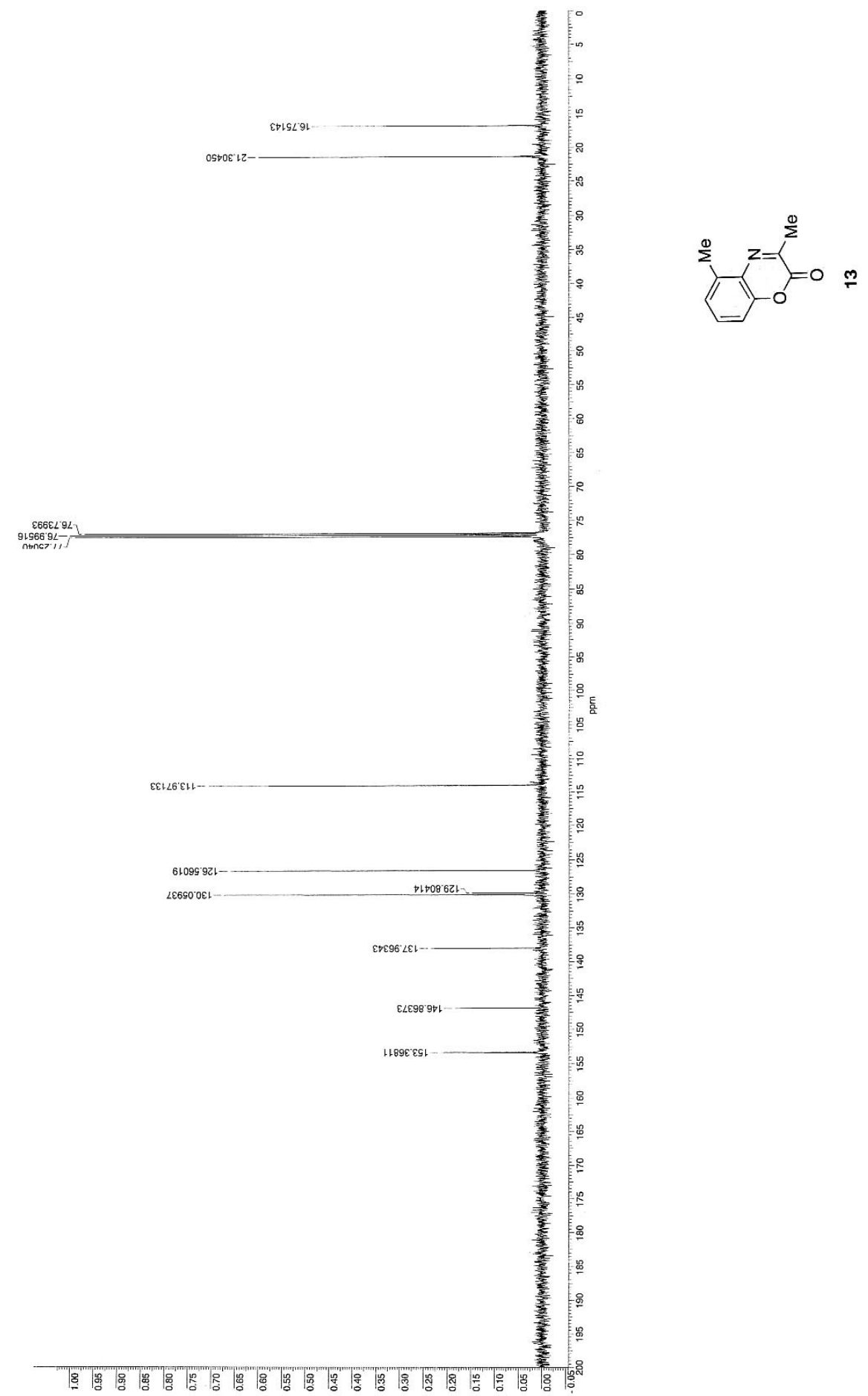




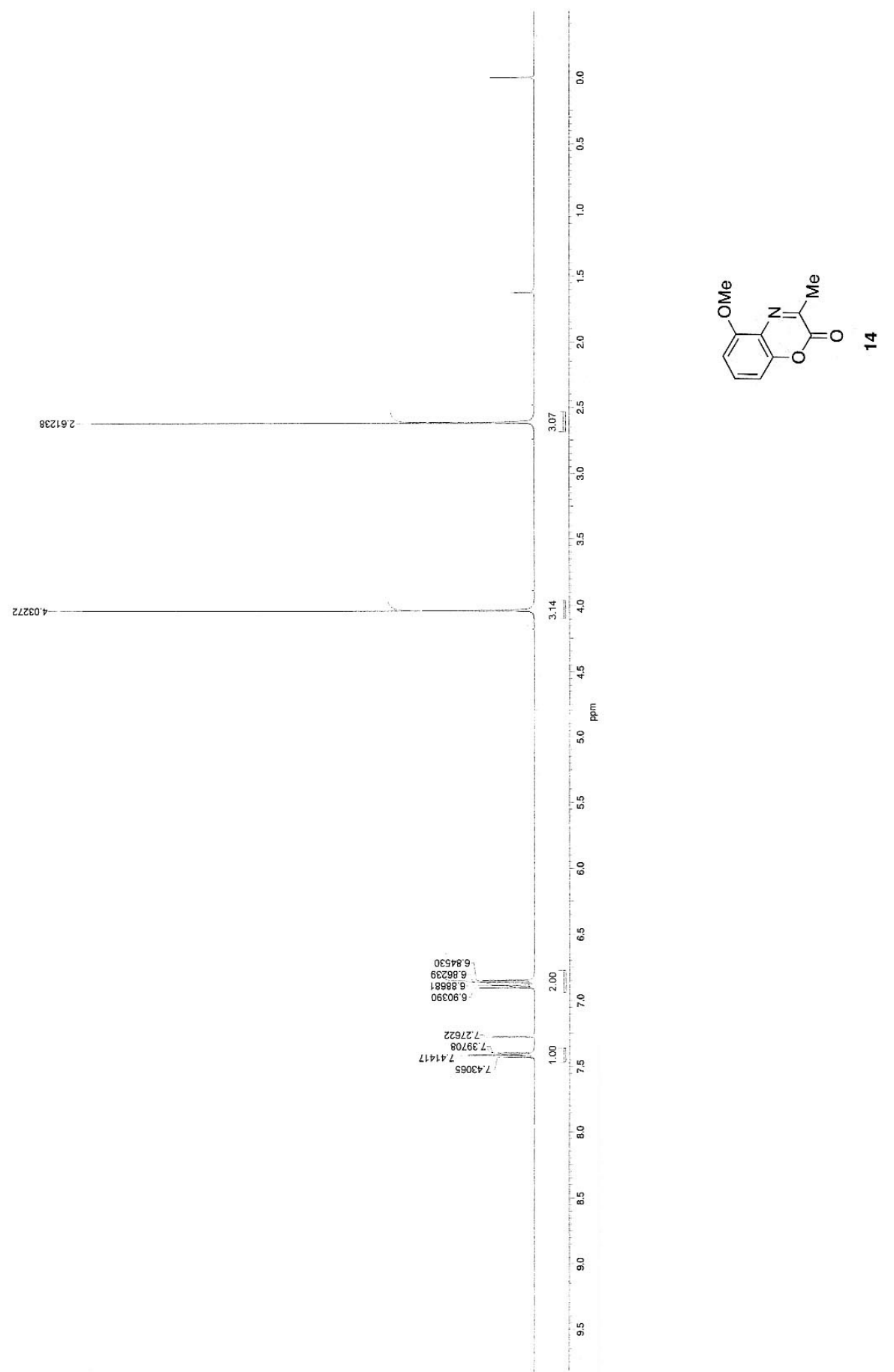

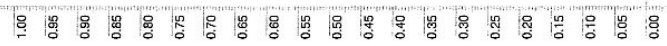




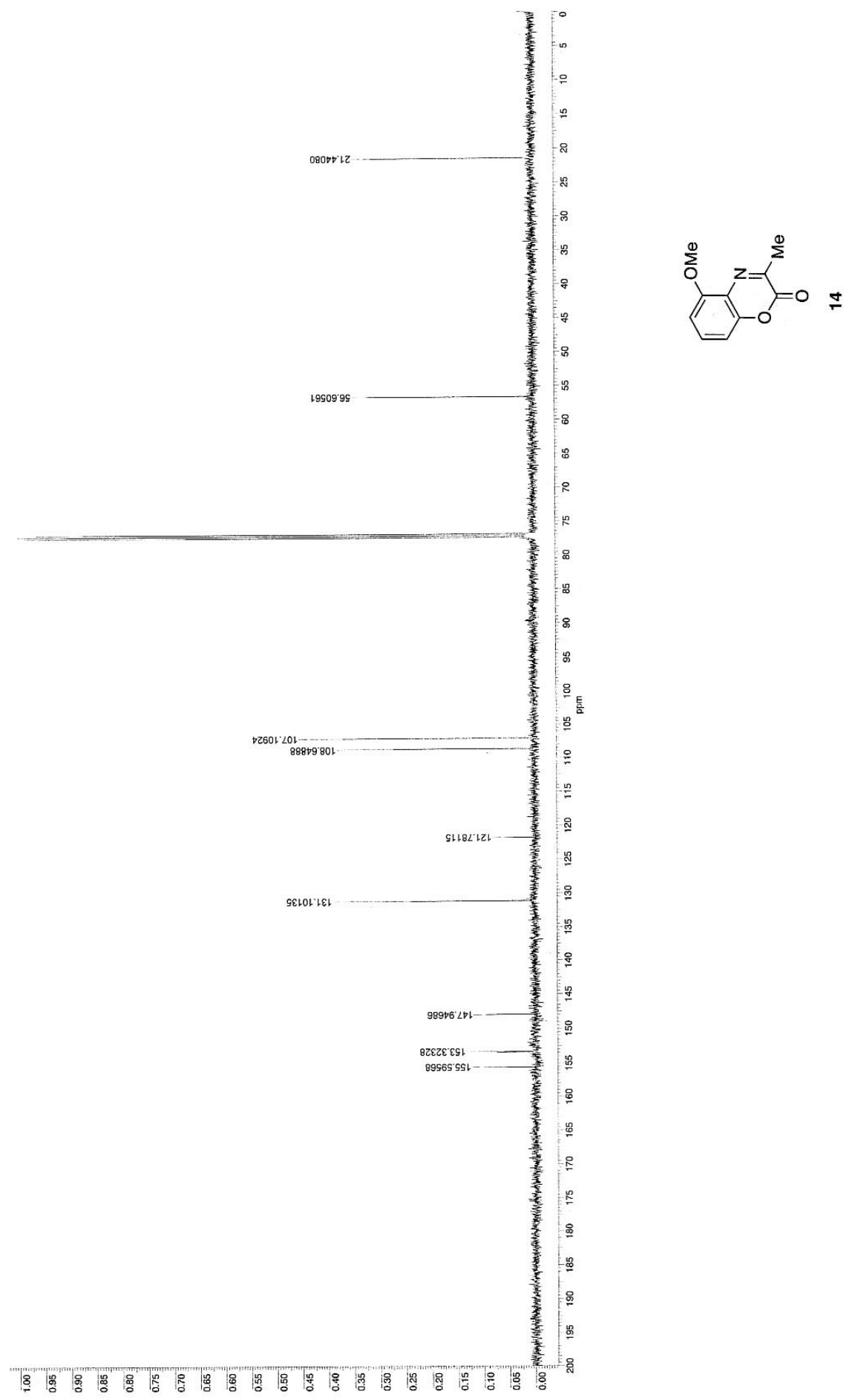




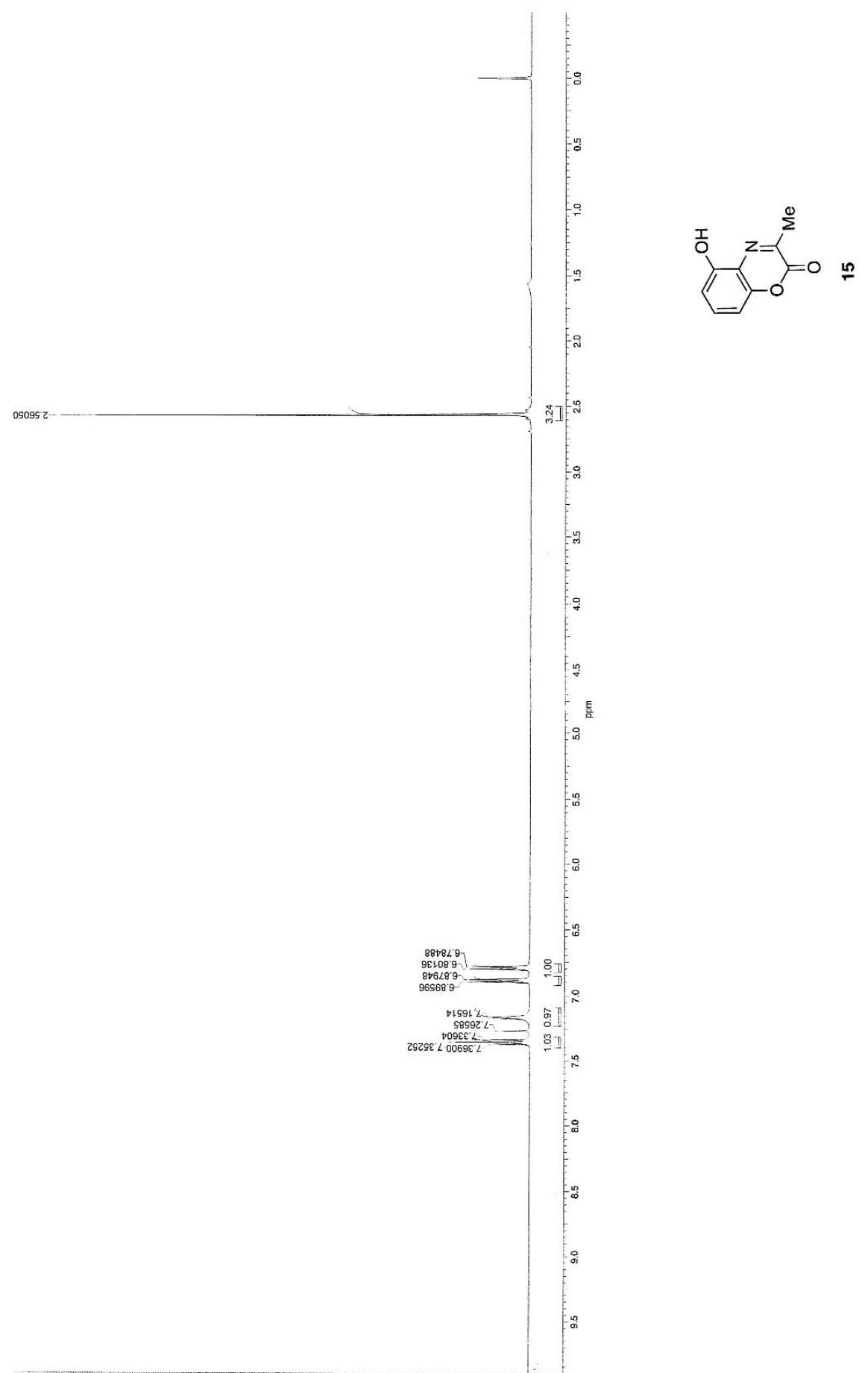

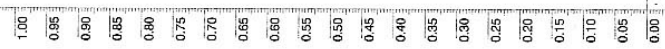

S30 


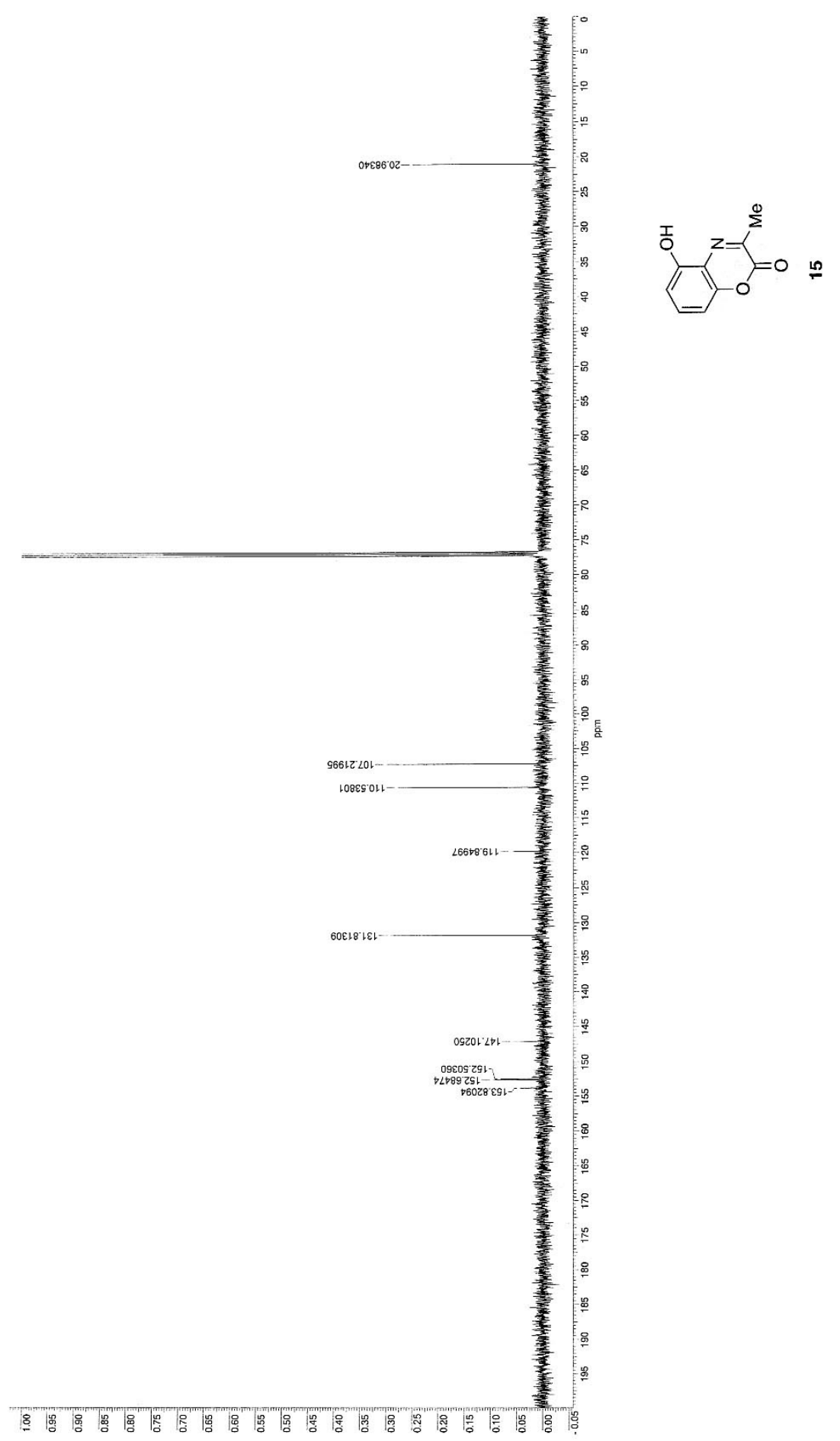

S31 


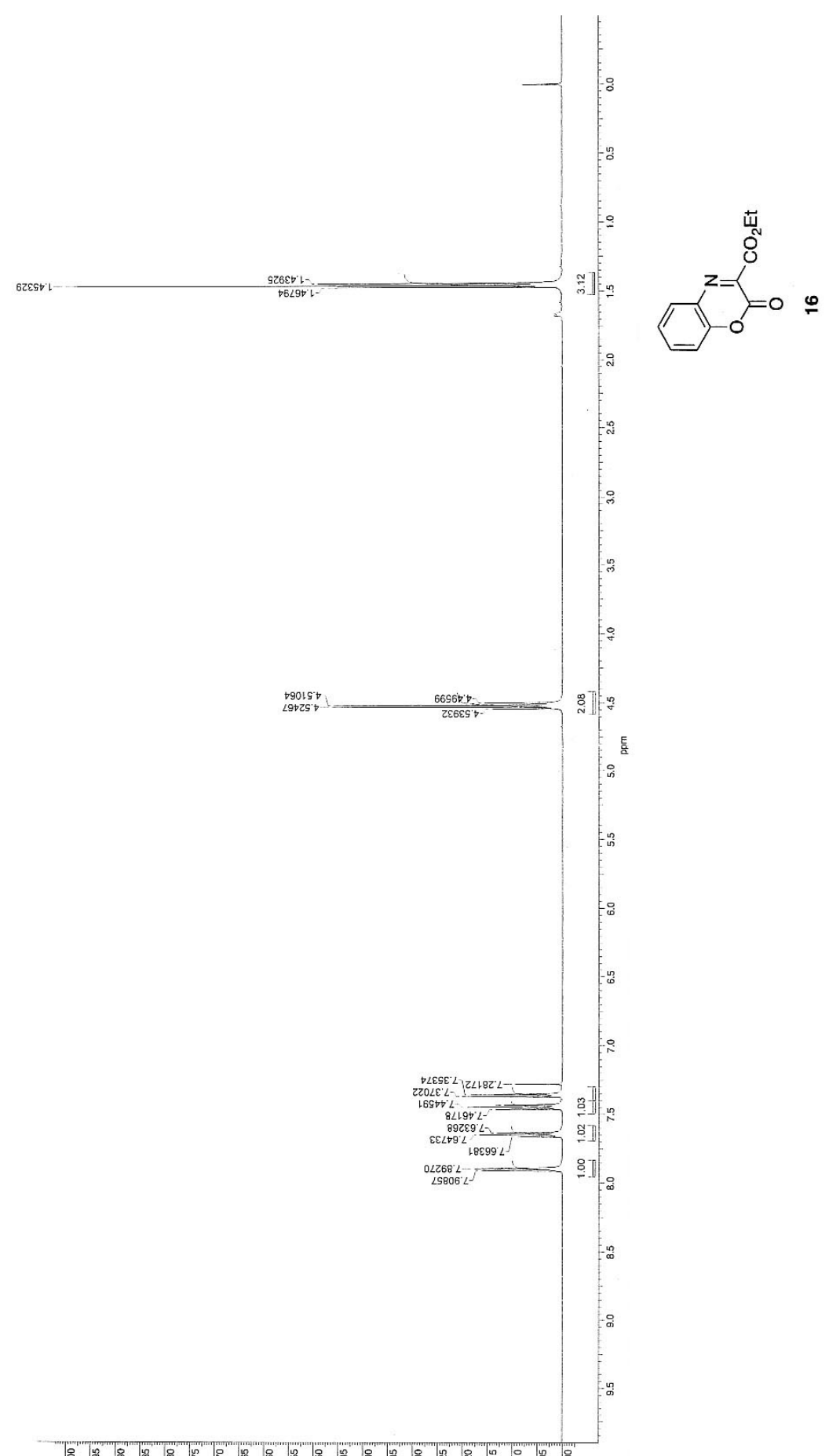

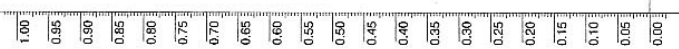




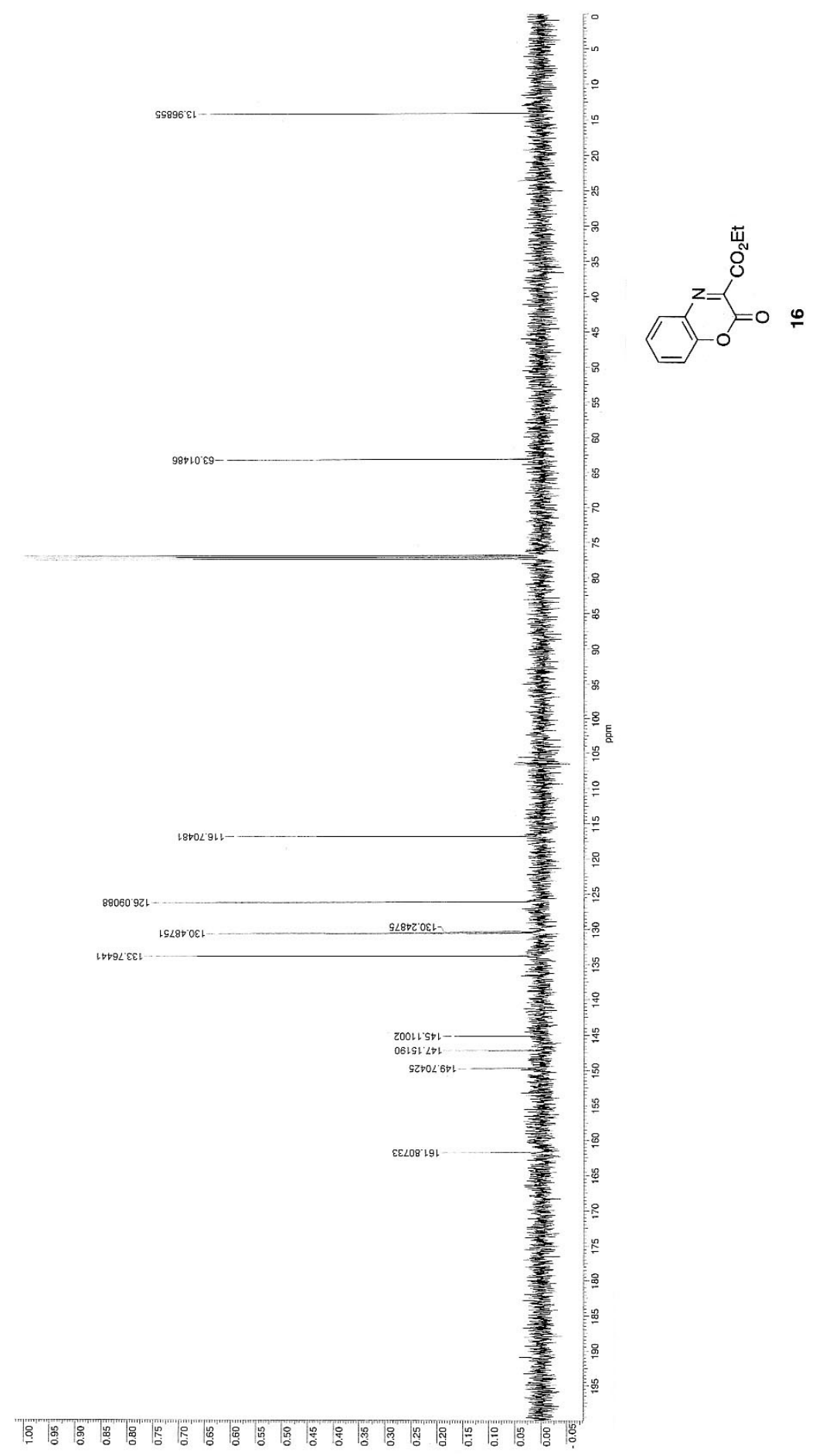

S33 


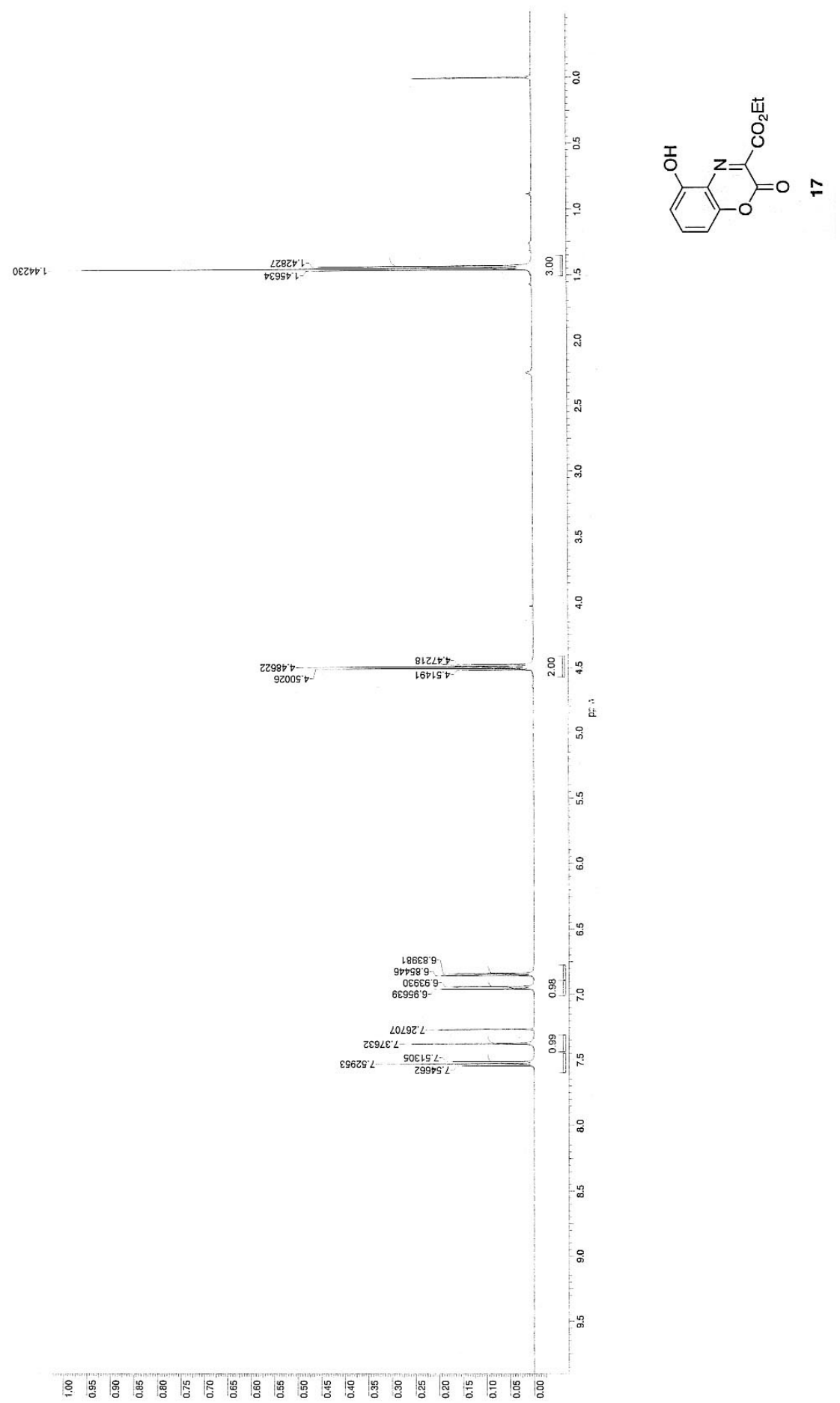

S34 


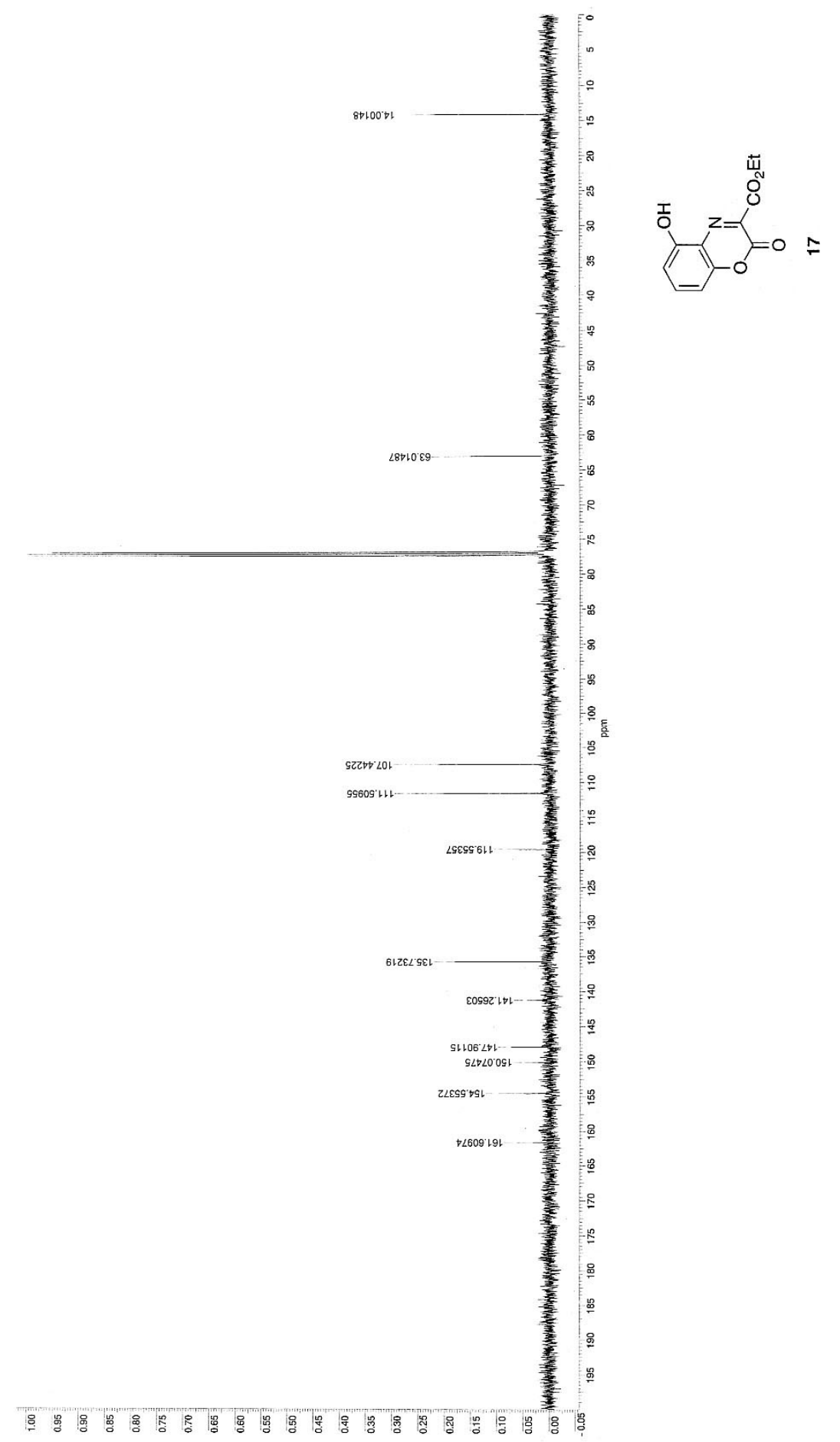

S35 


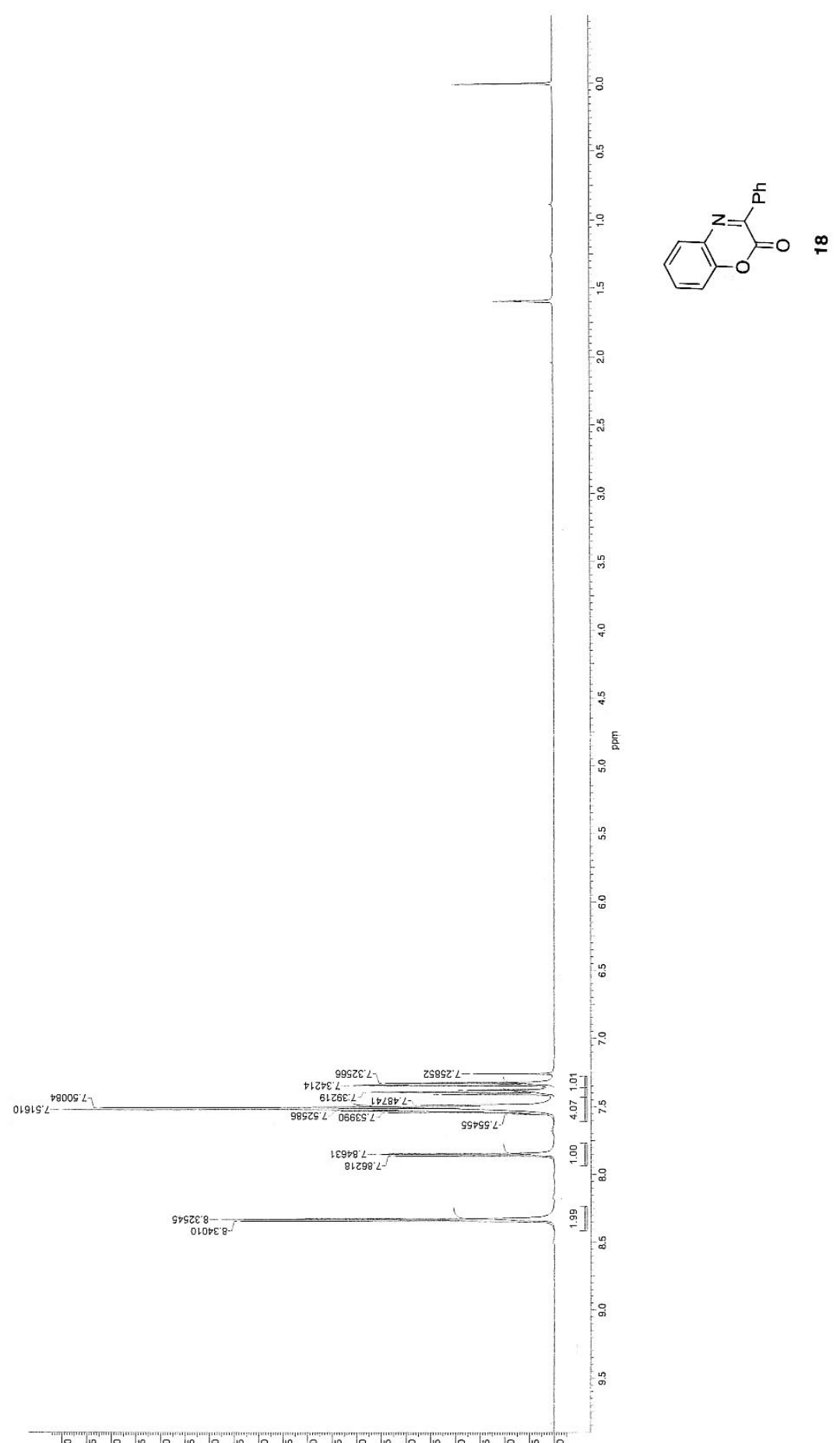

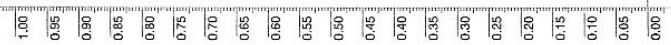




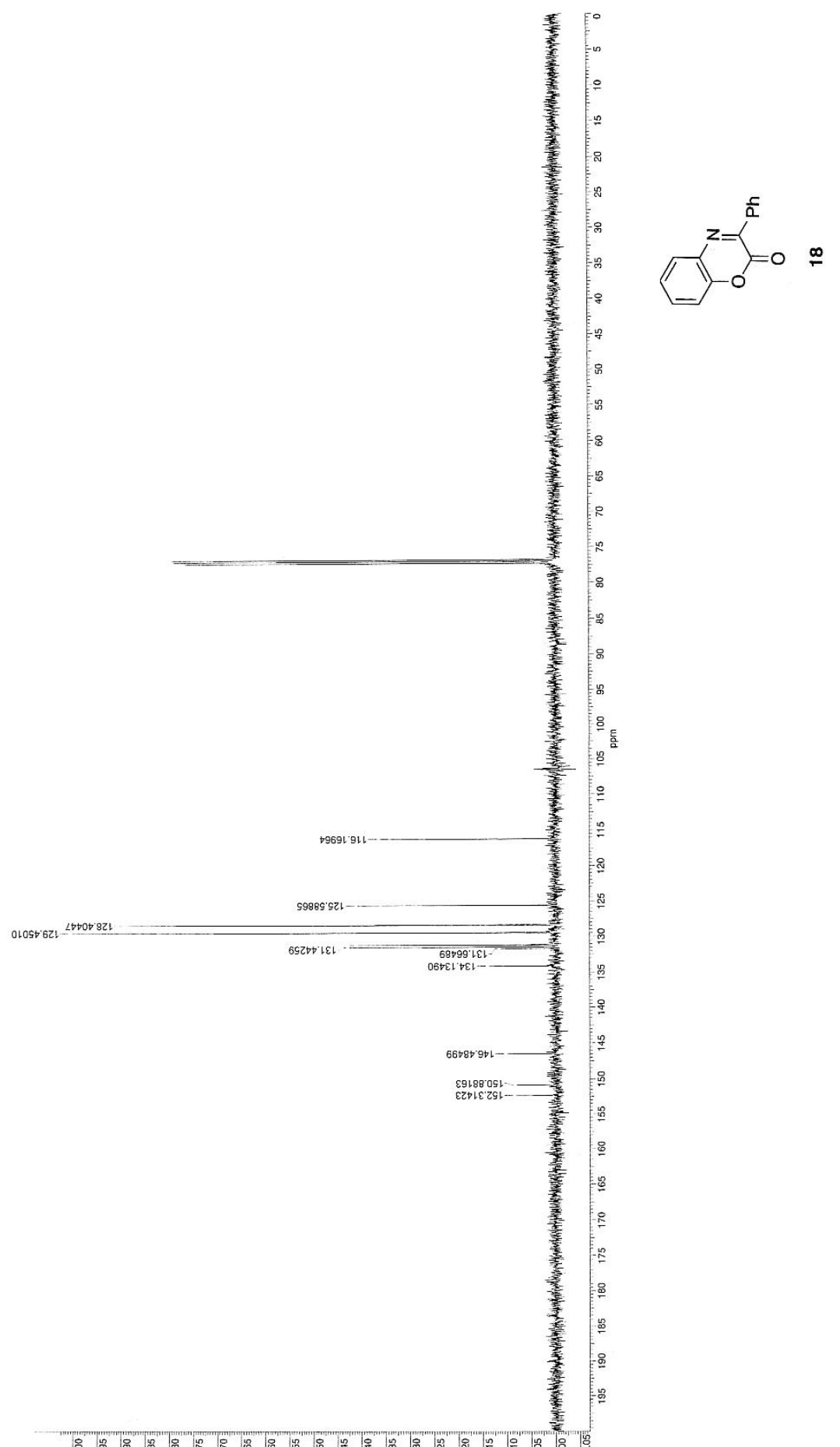

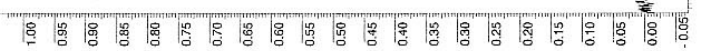




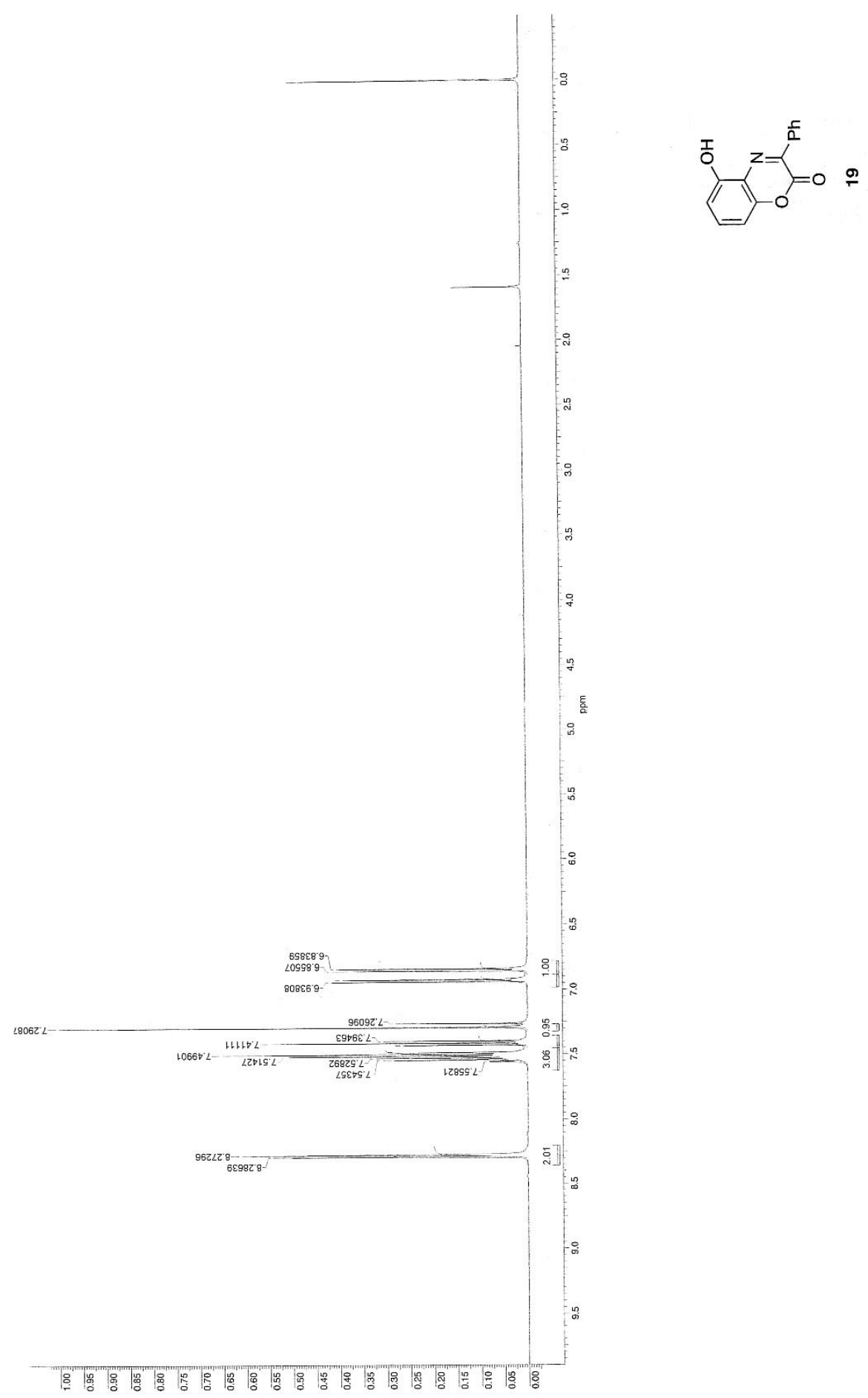

S38 


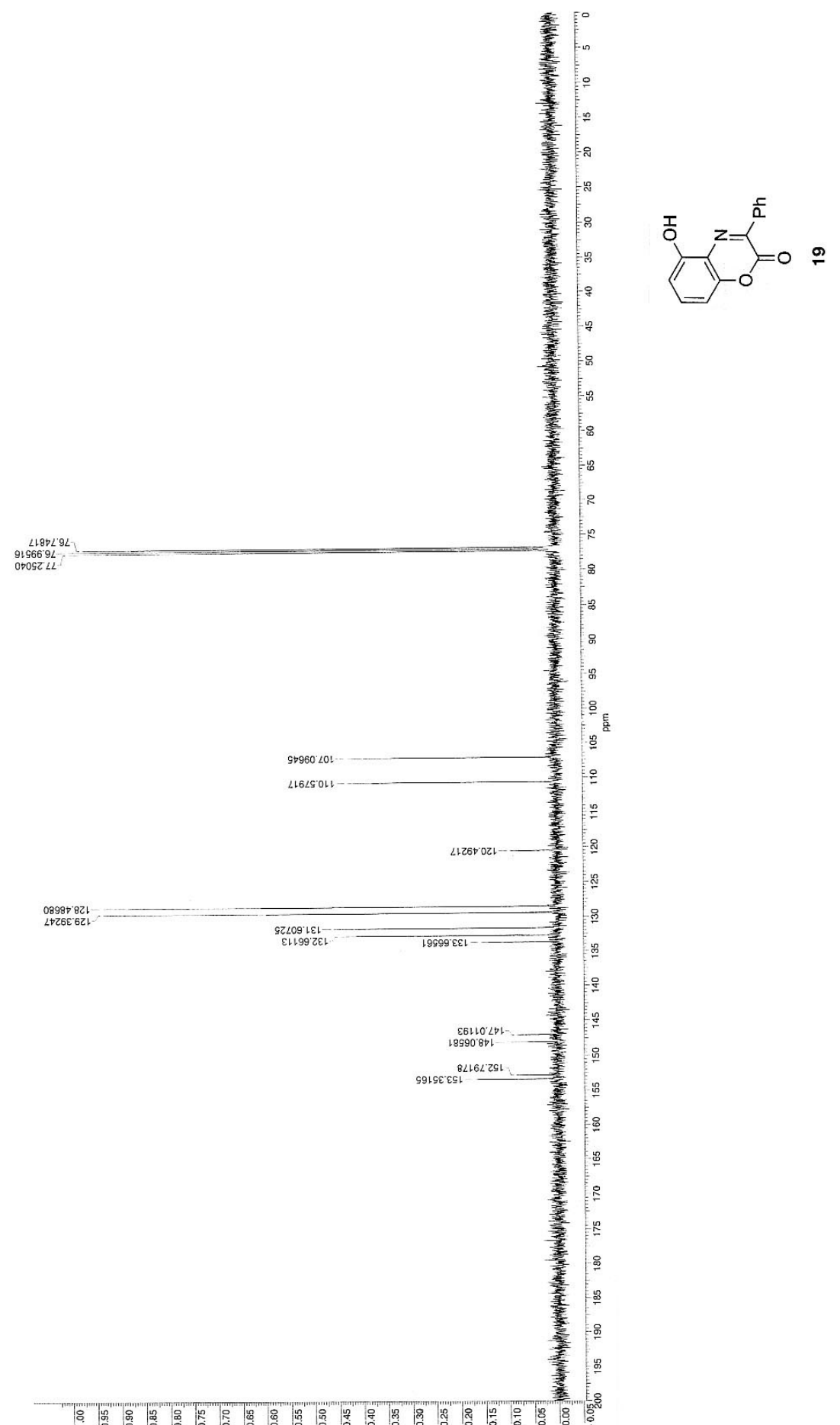

S39 


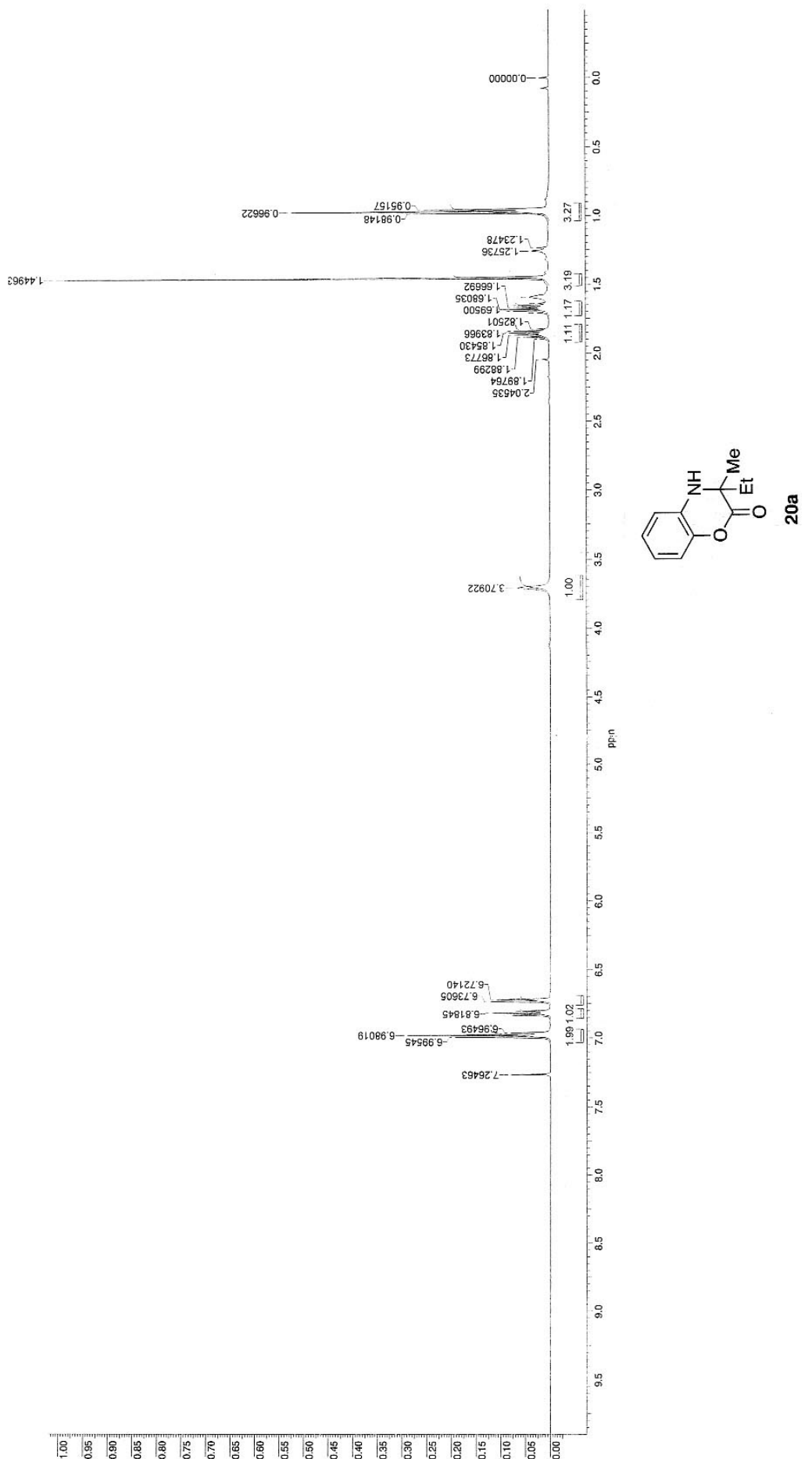

$\mathrm{S} 40$ 


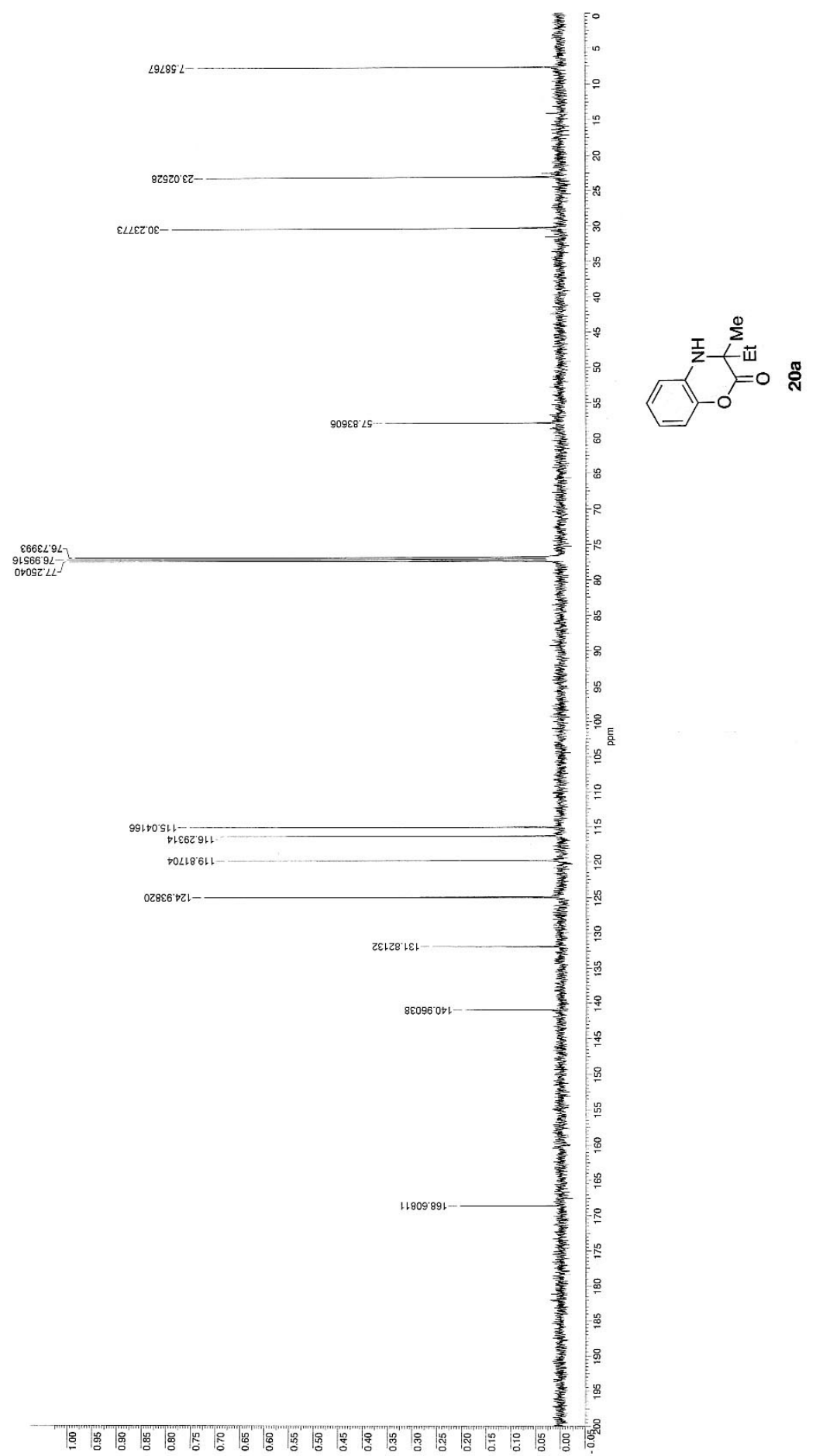

S41 


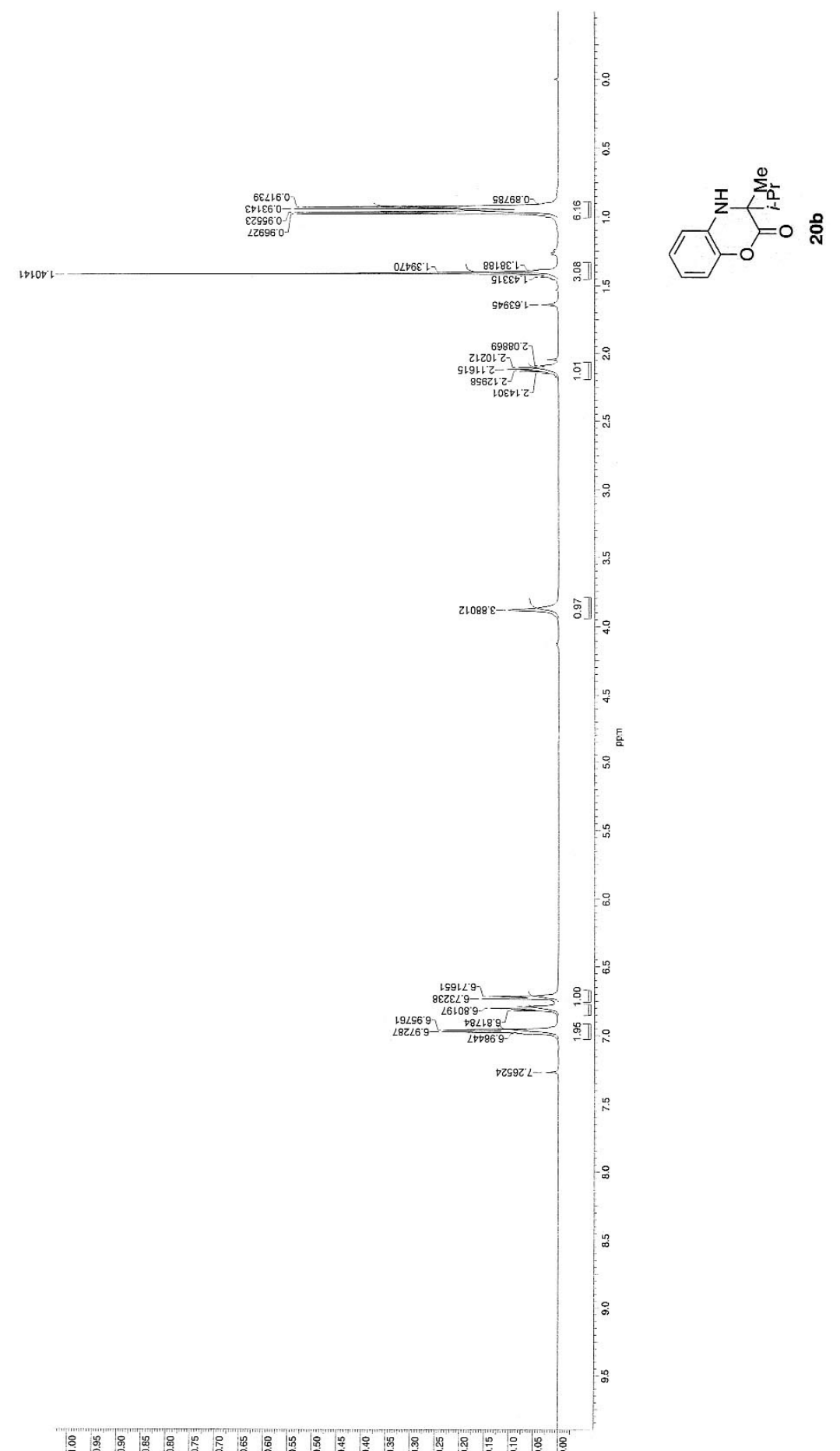

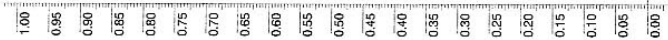




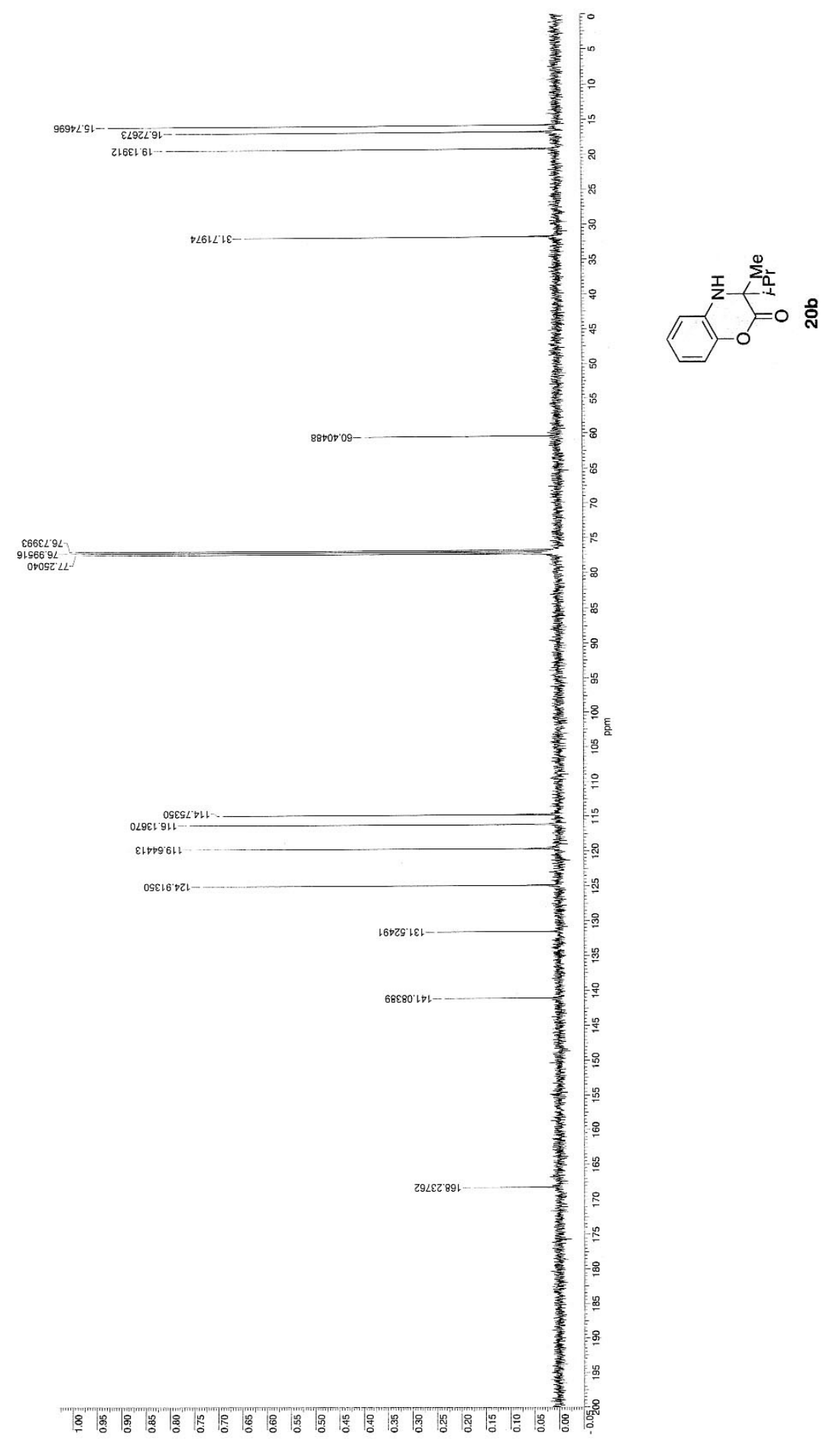

$\mathrm{S} 43$ 


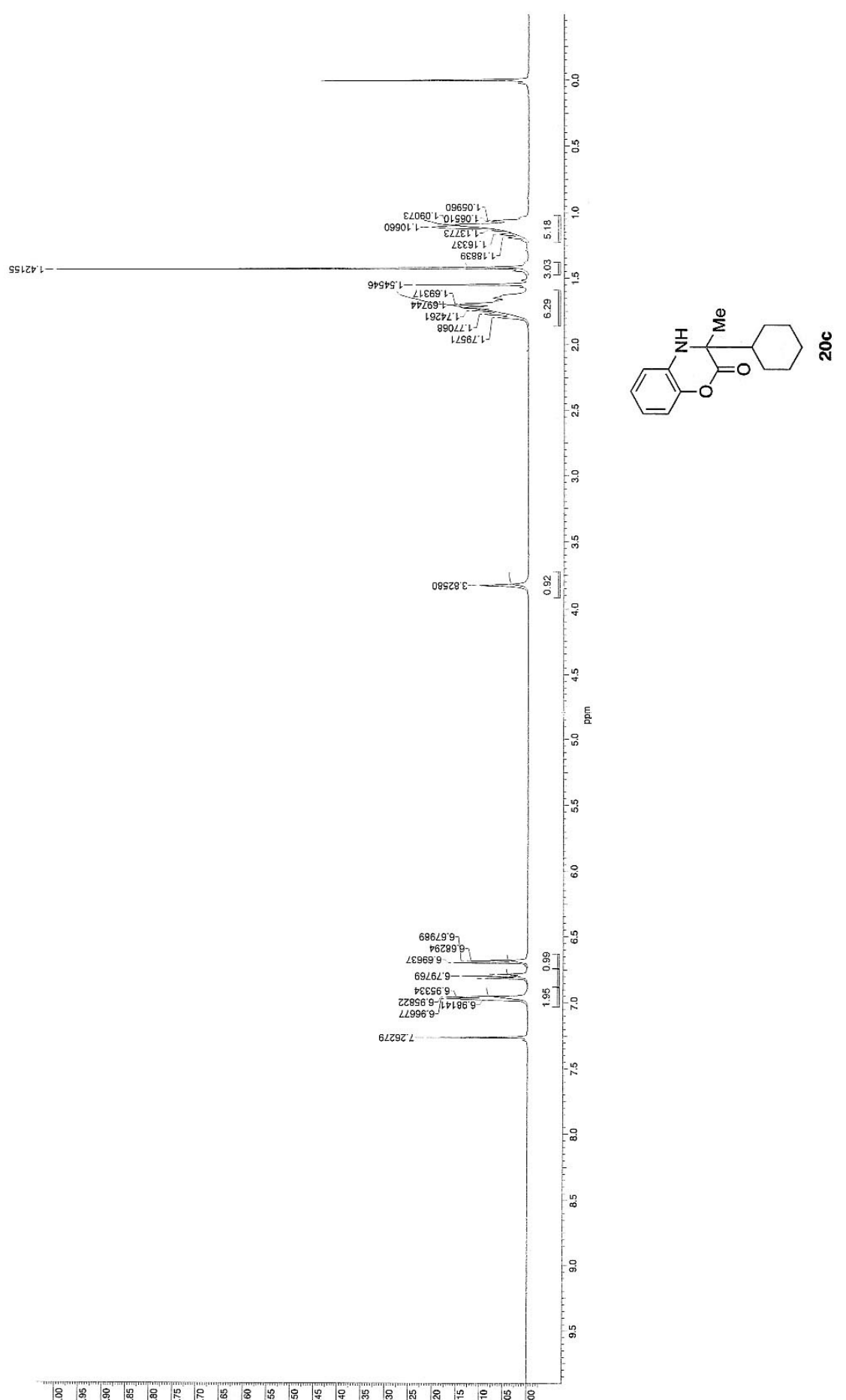

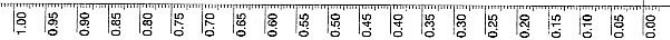




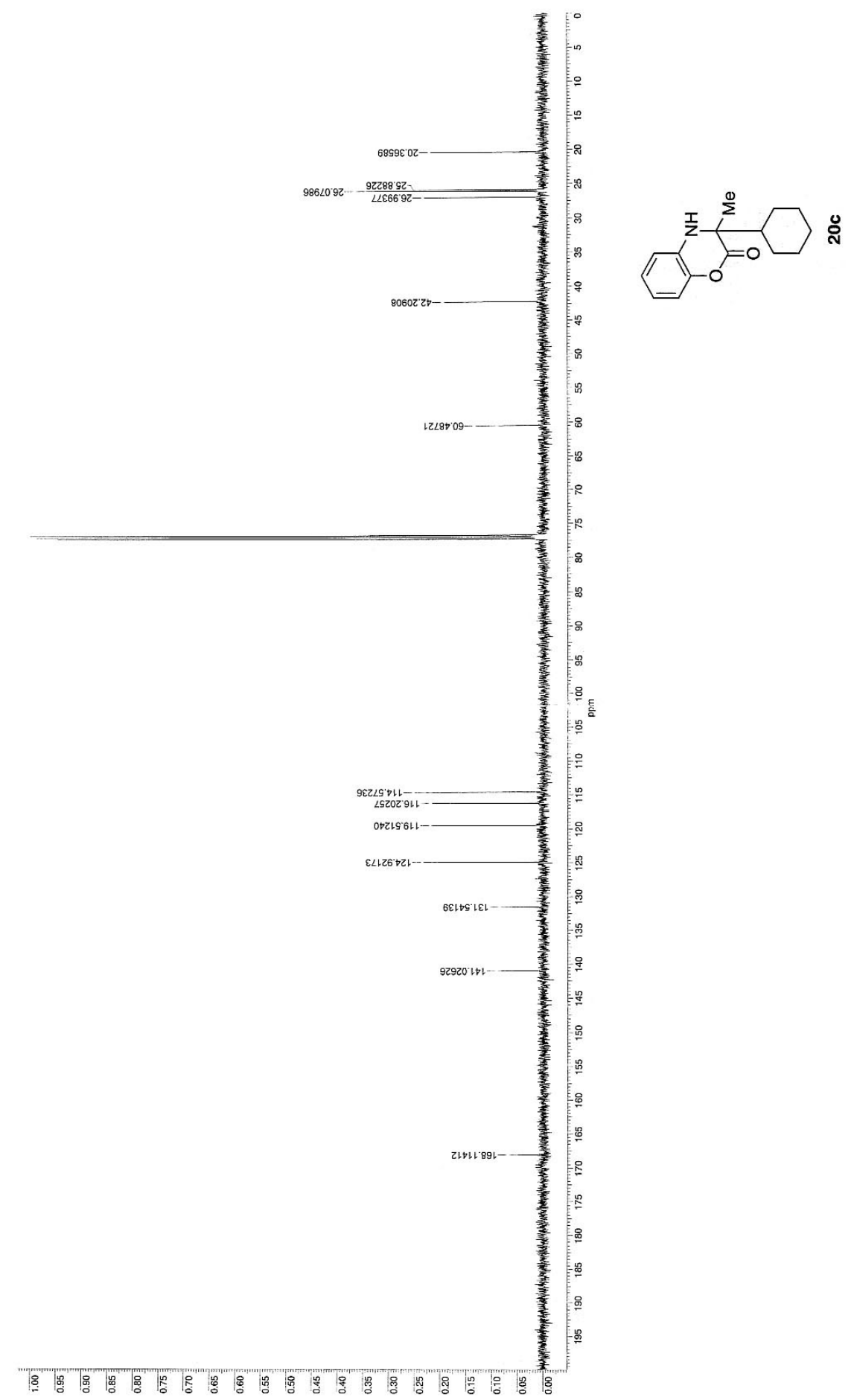




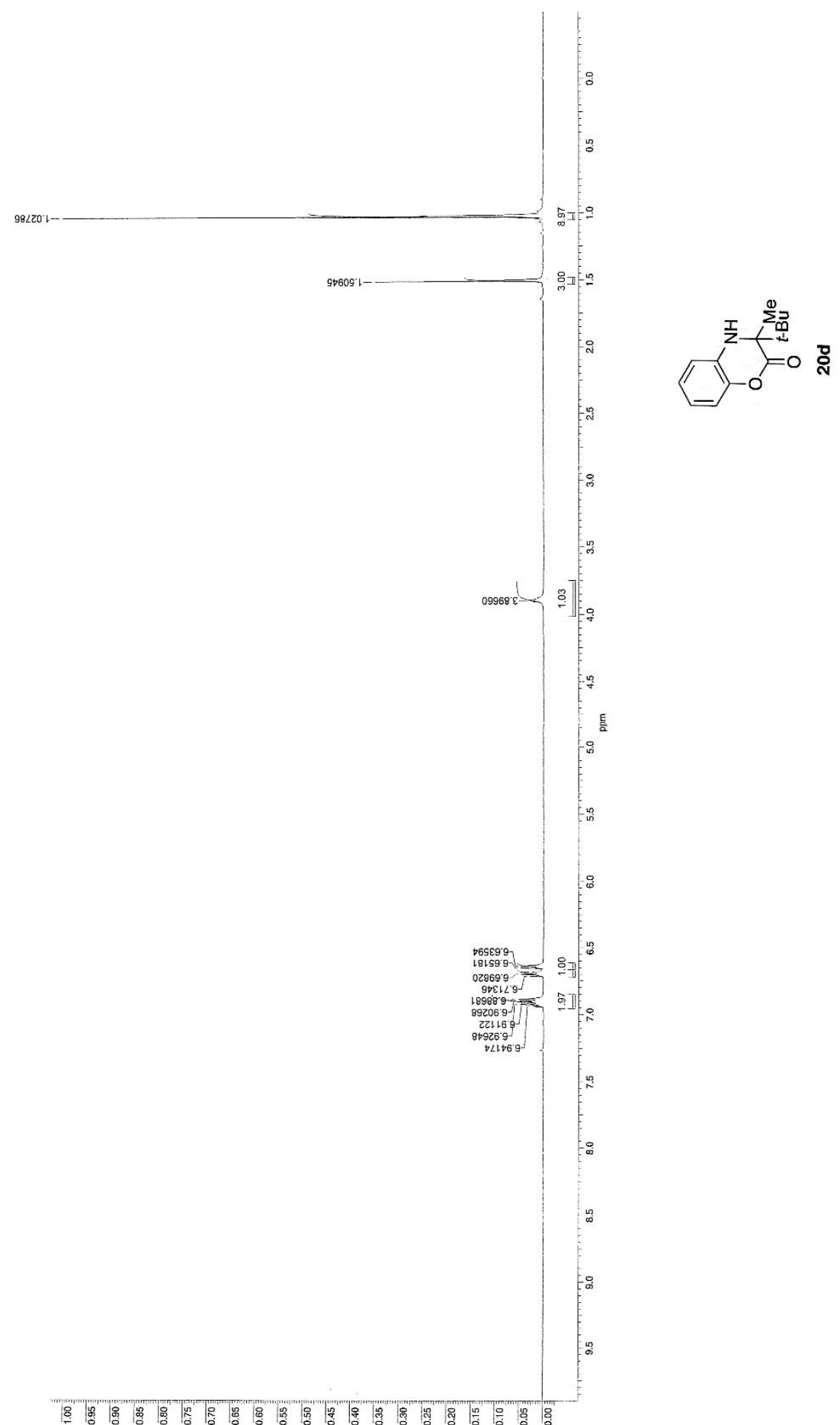




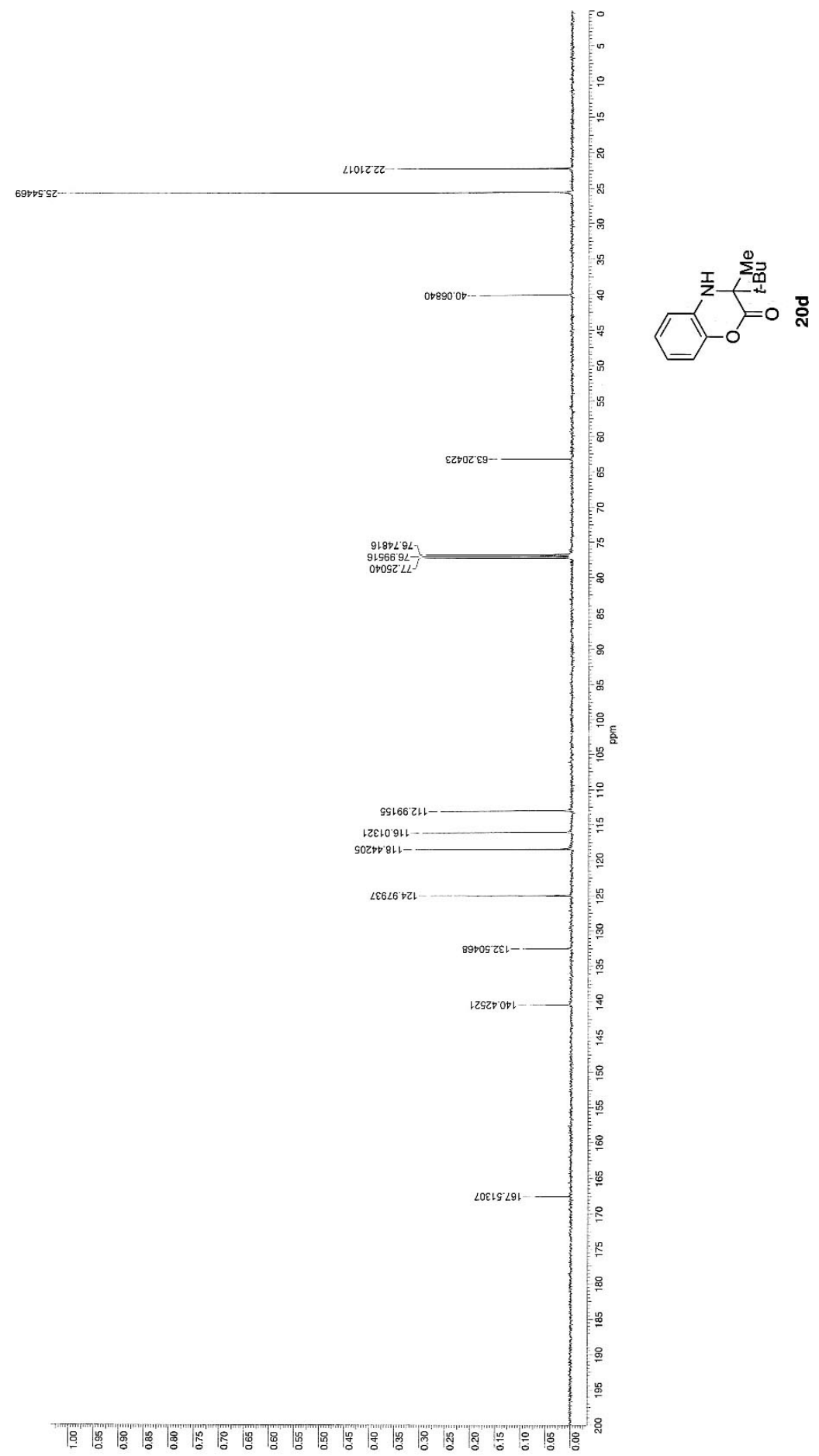




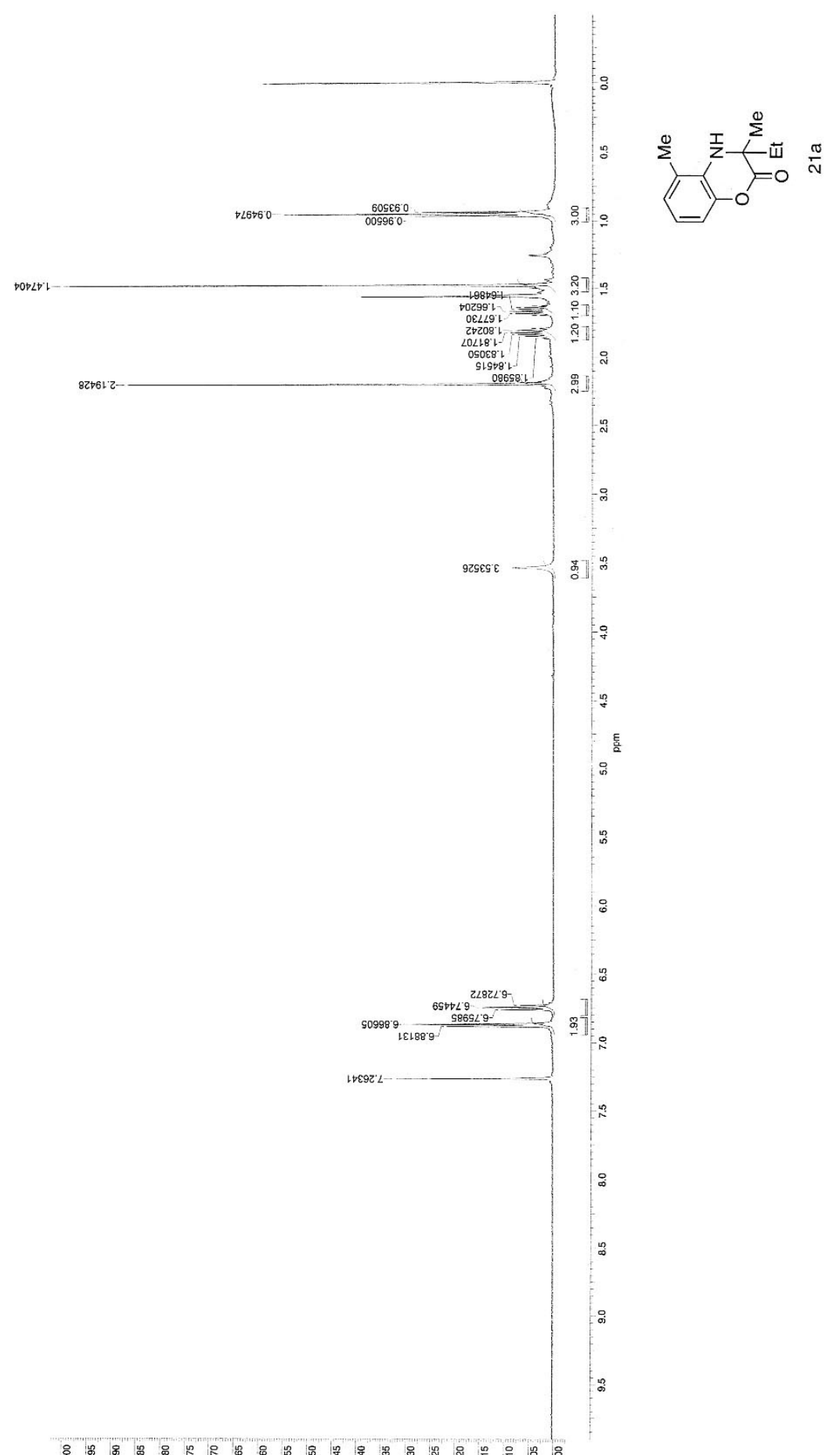

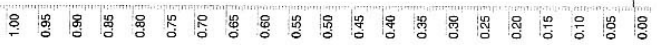




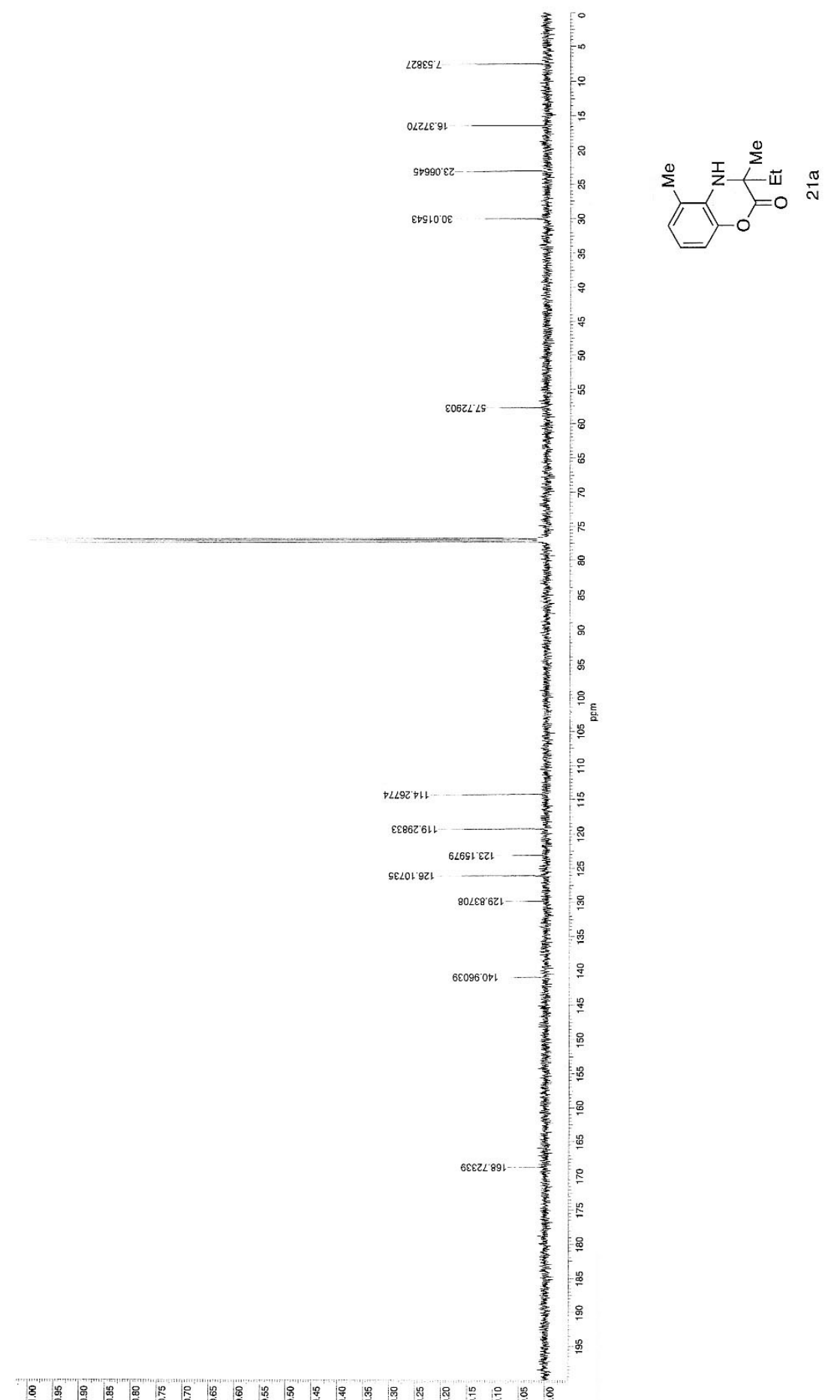

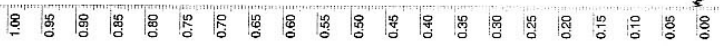




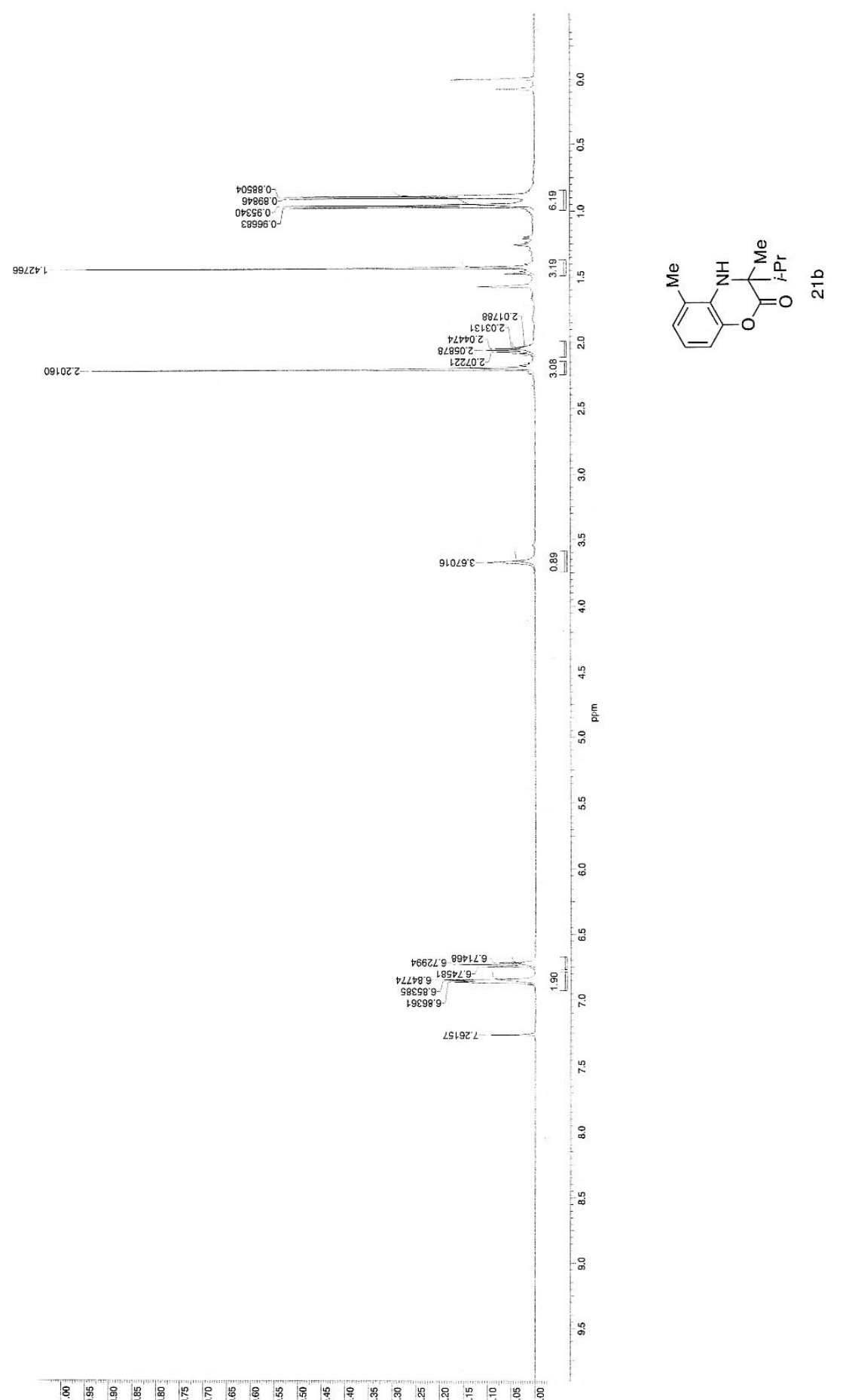

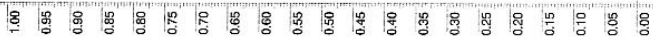

S50 


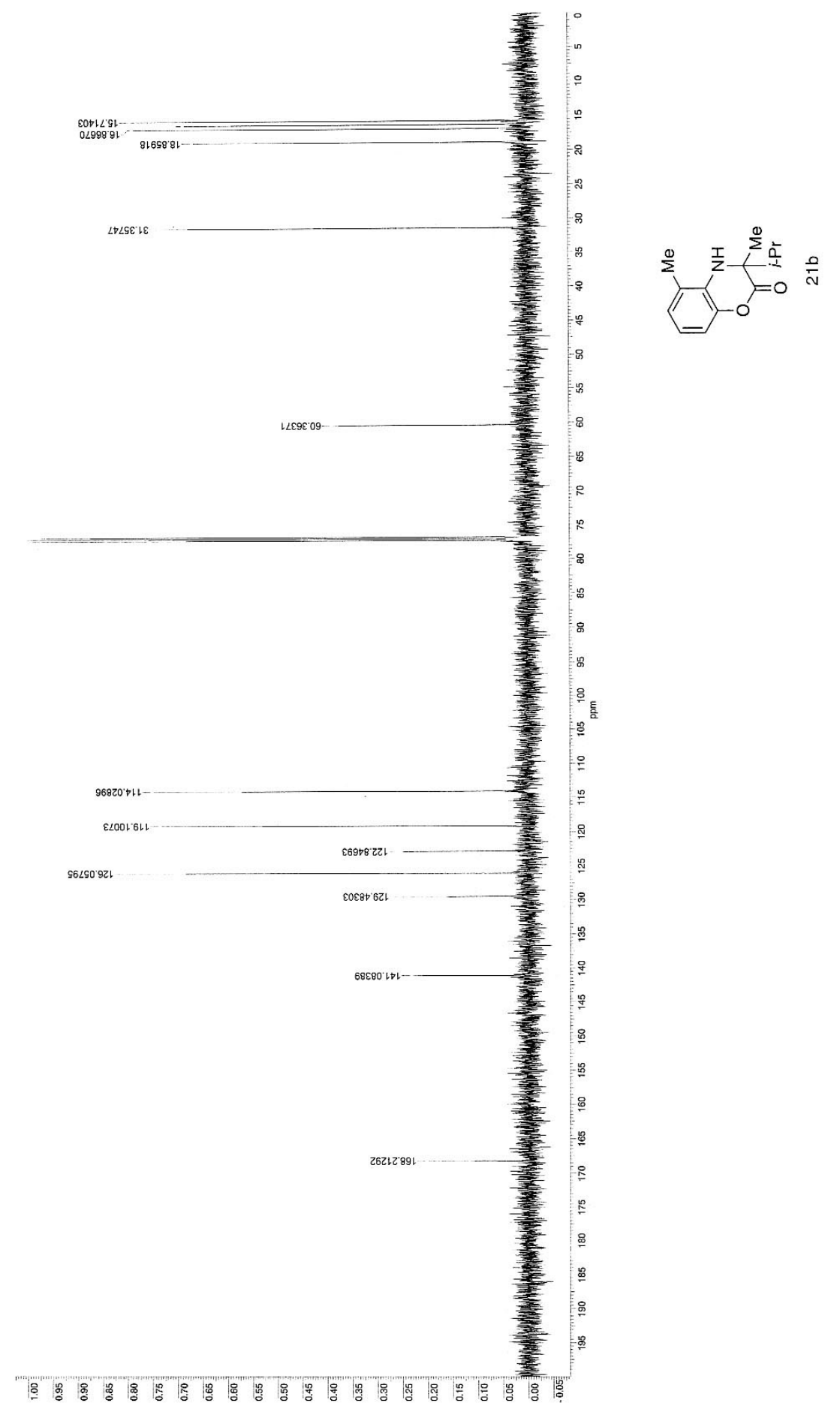

S51 


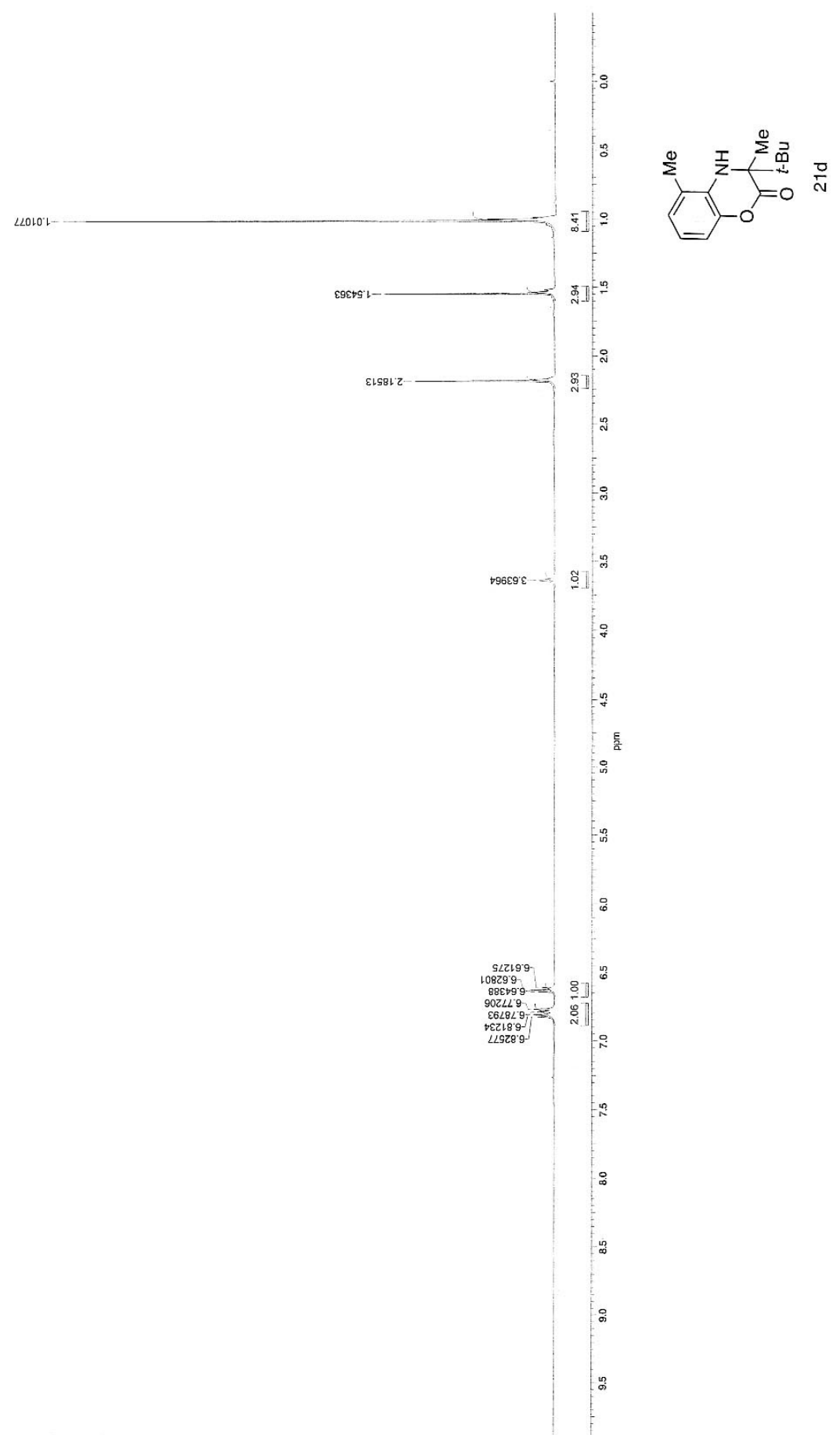

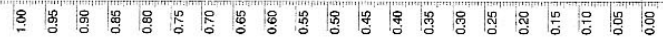




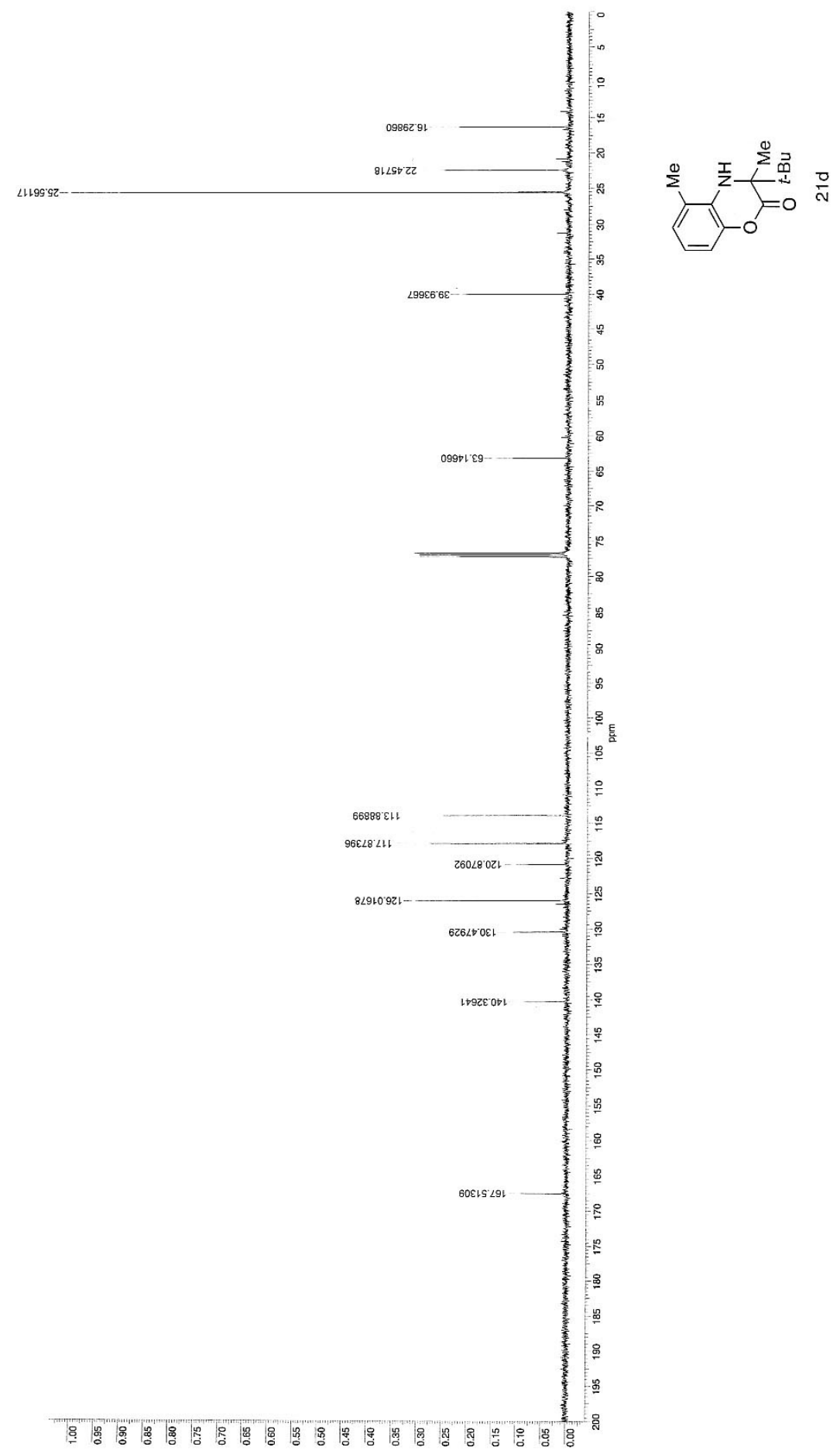




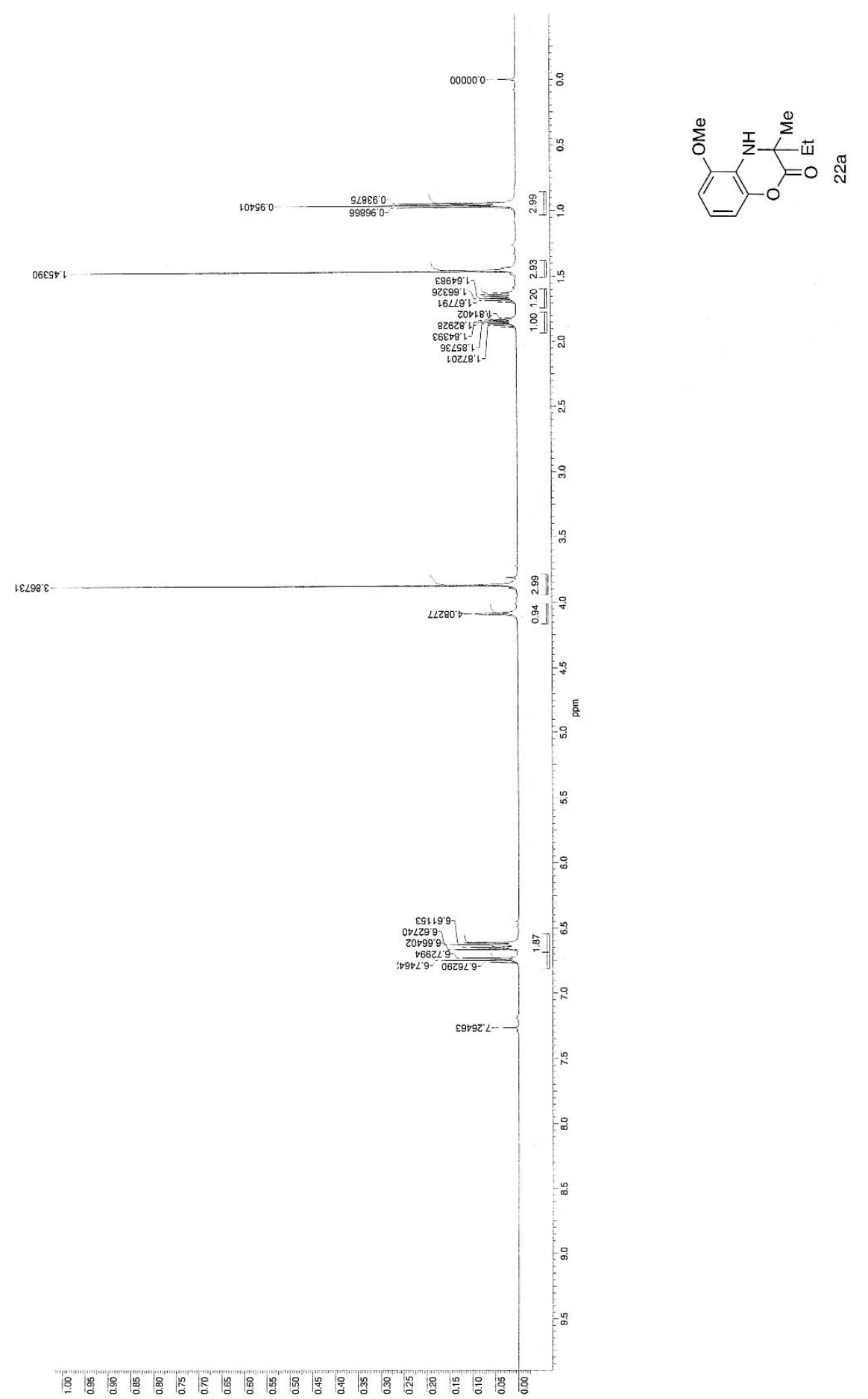

S54 


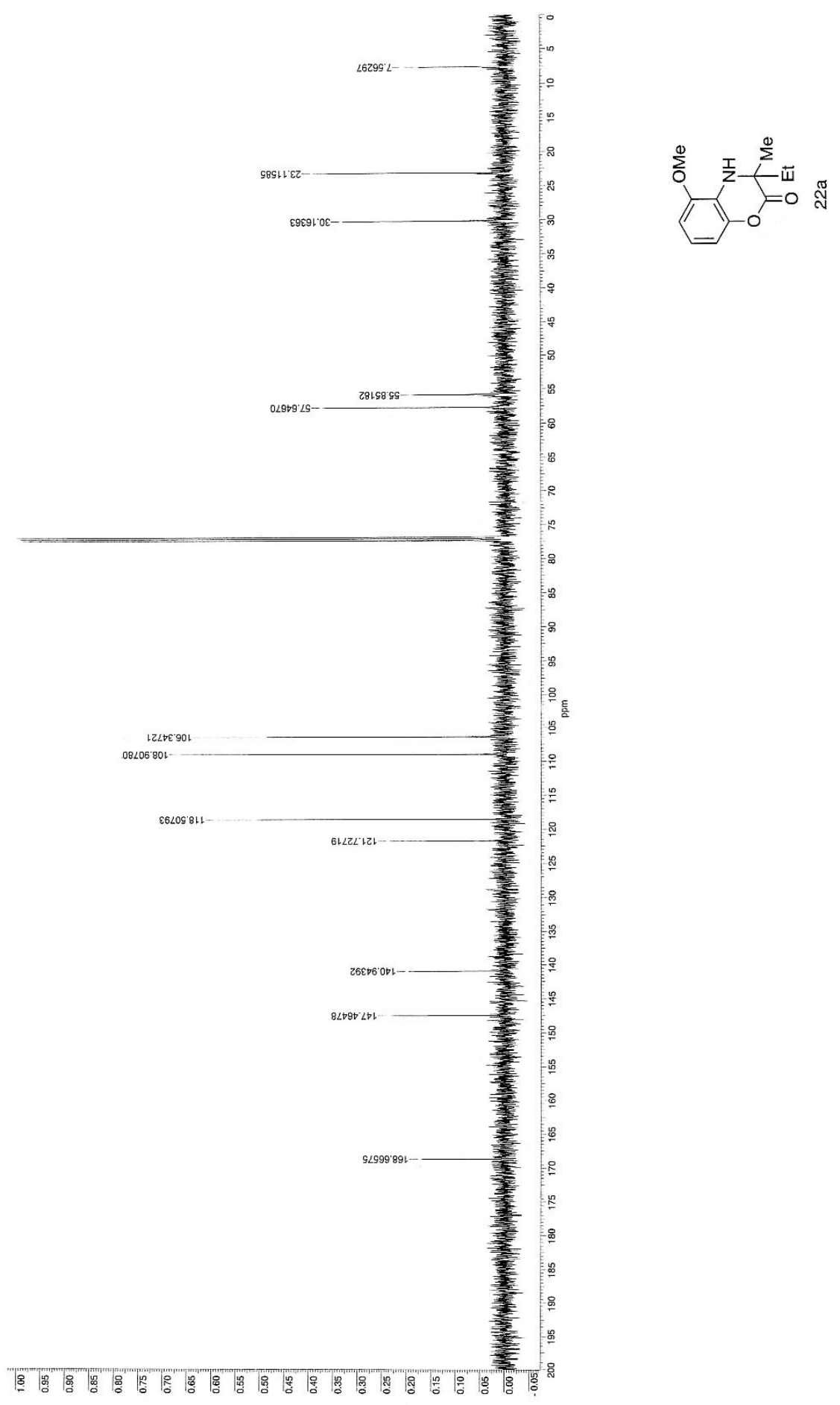

S55 


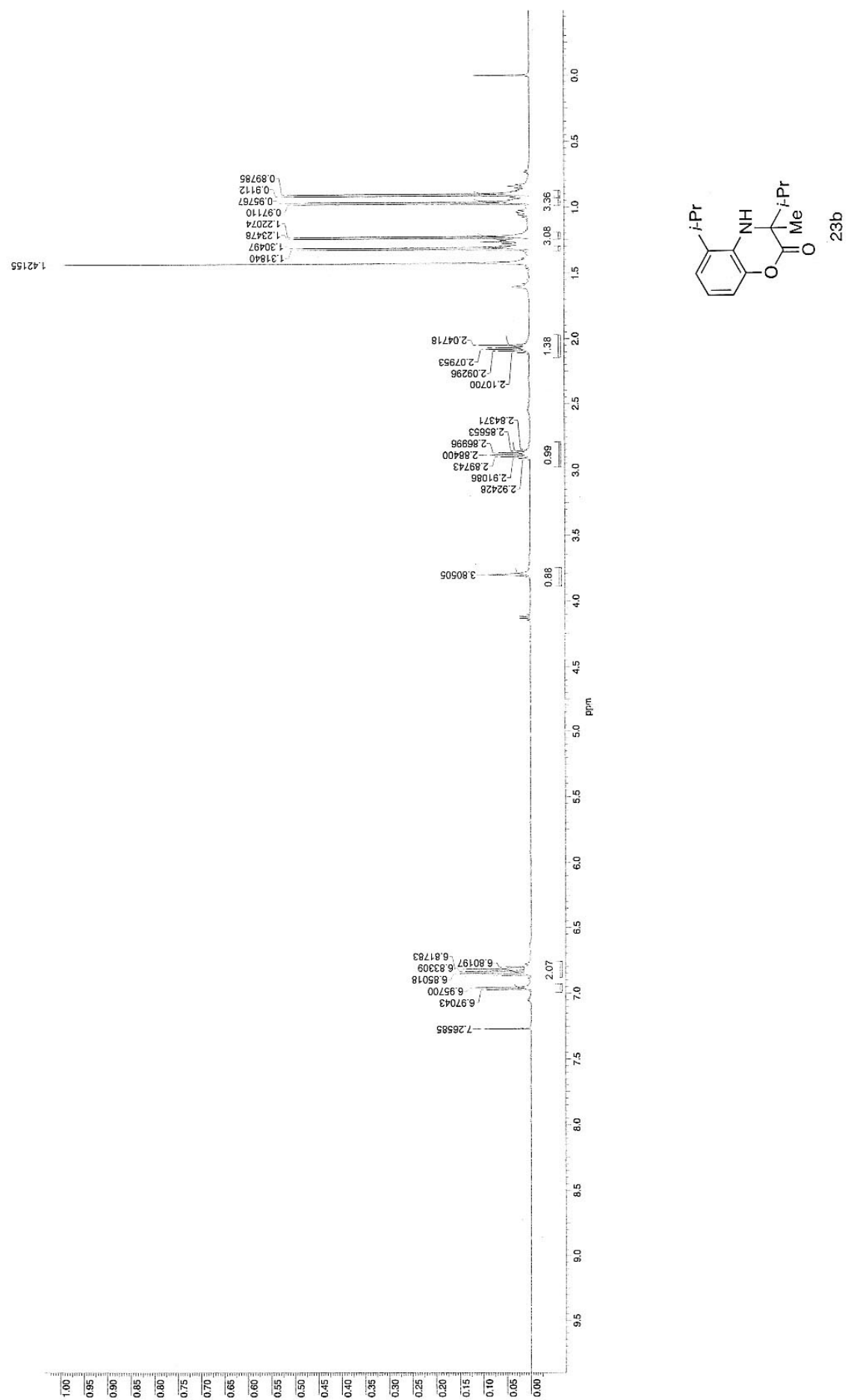

S56 


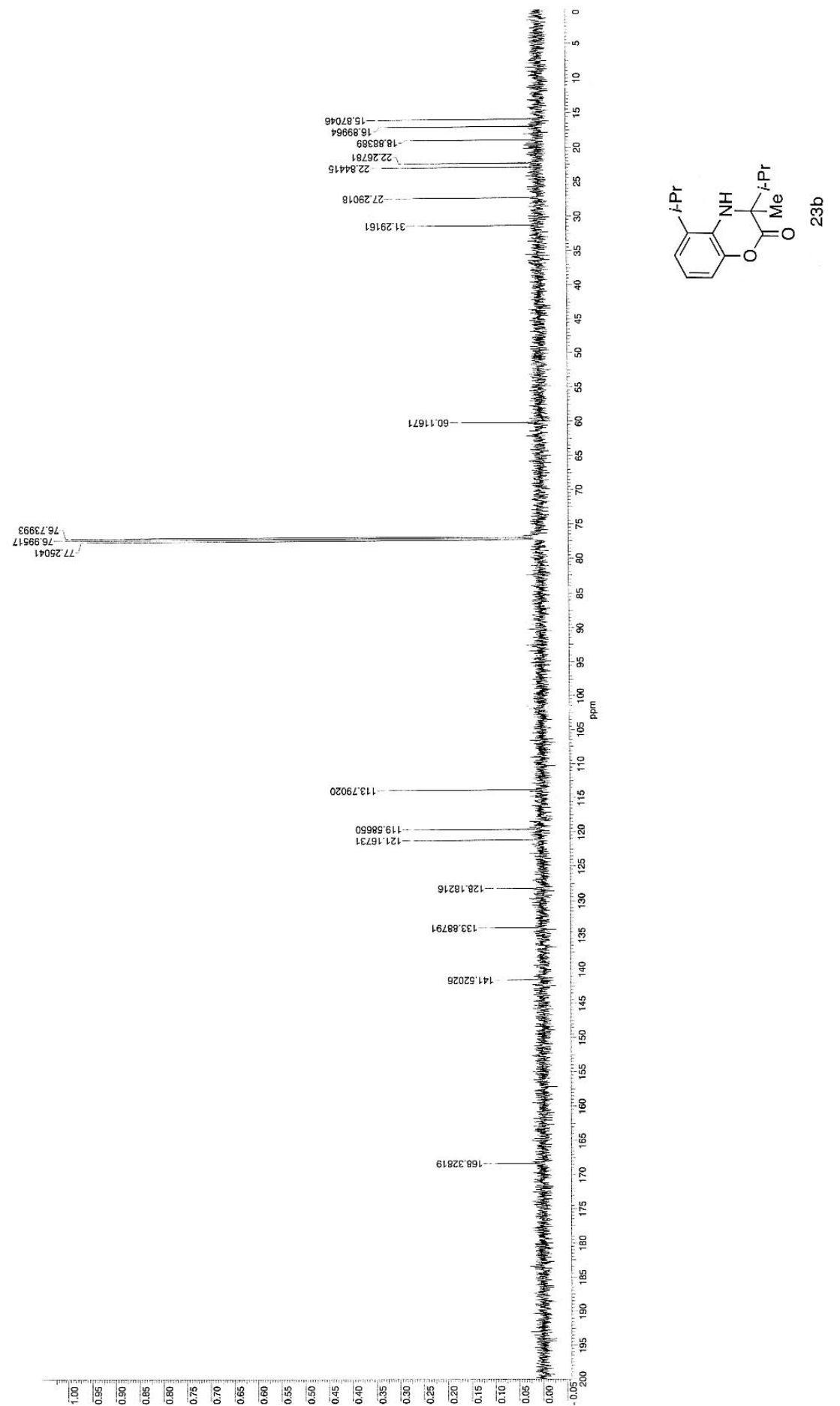

S57 


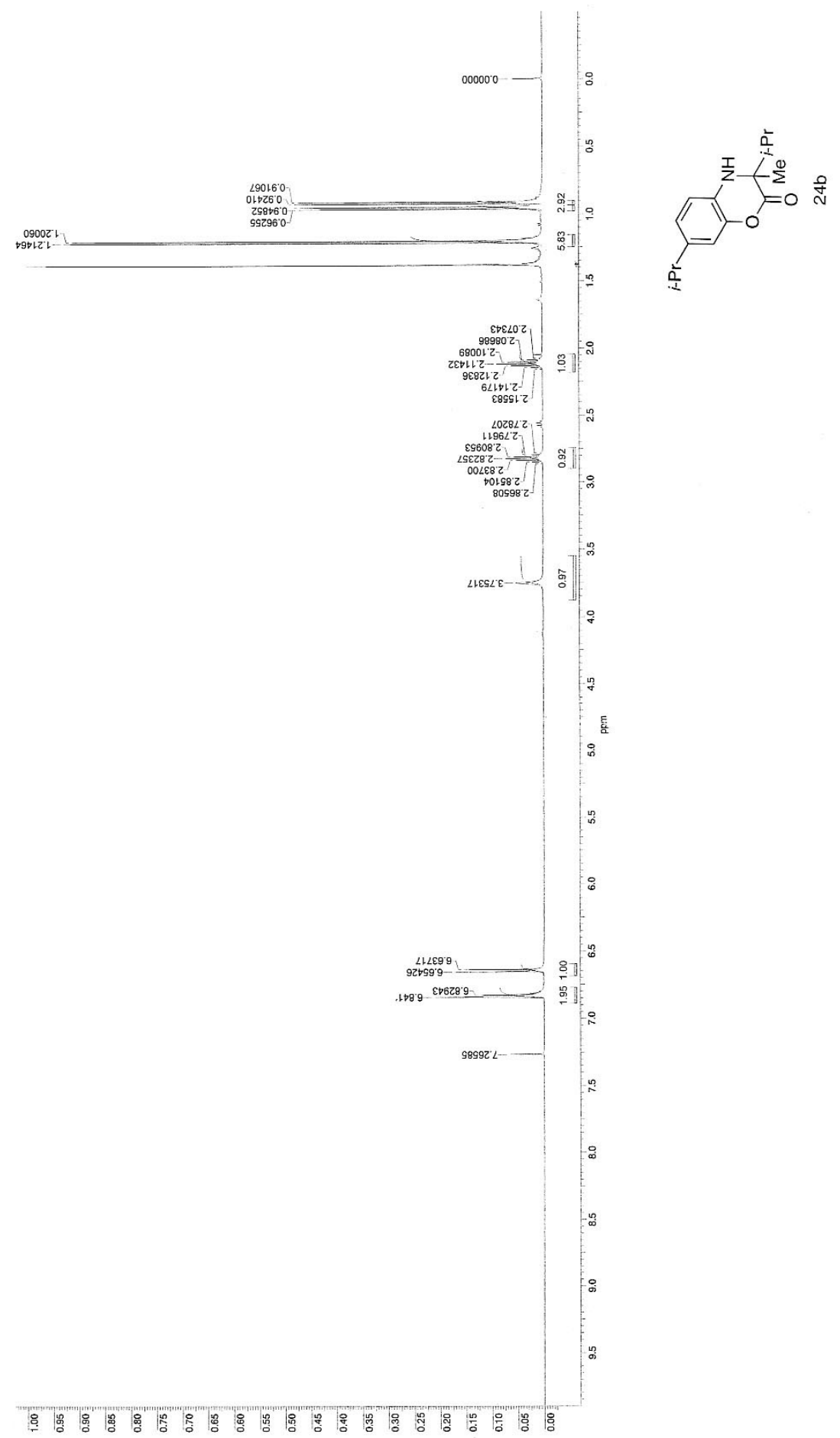

S58 


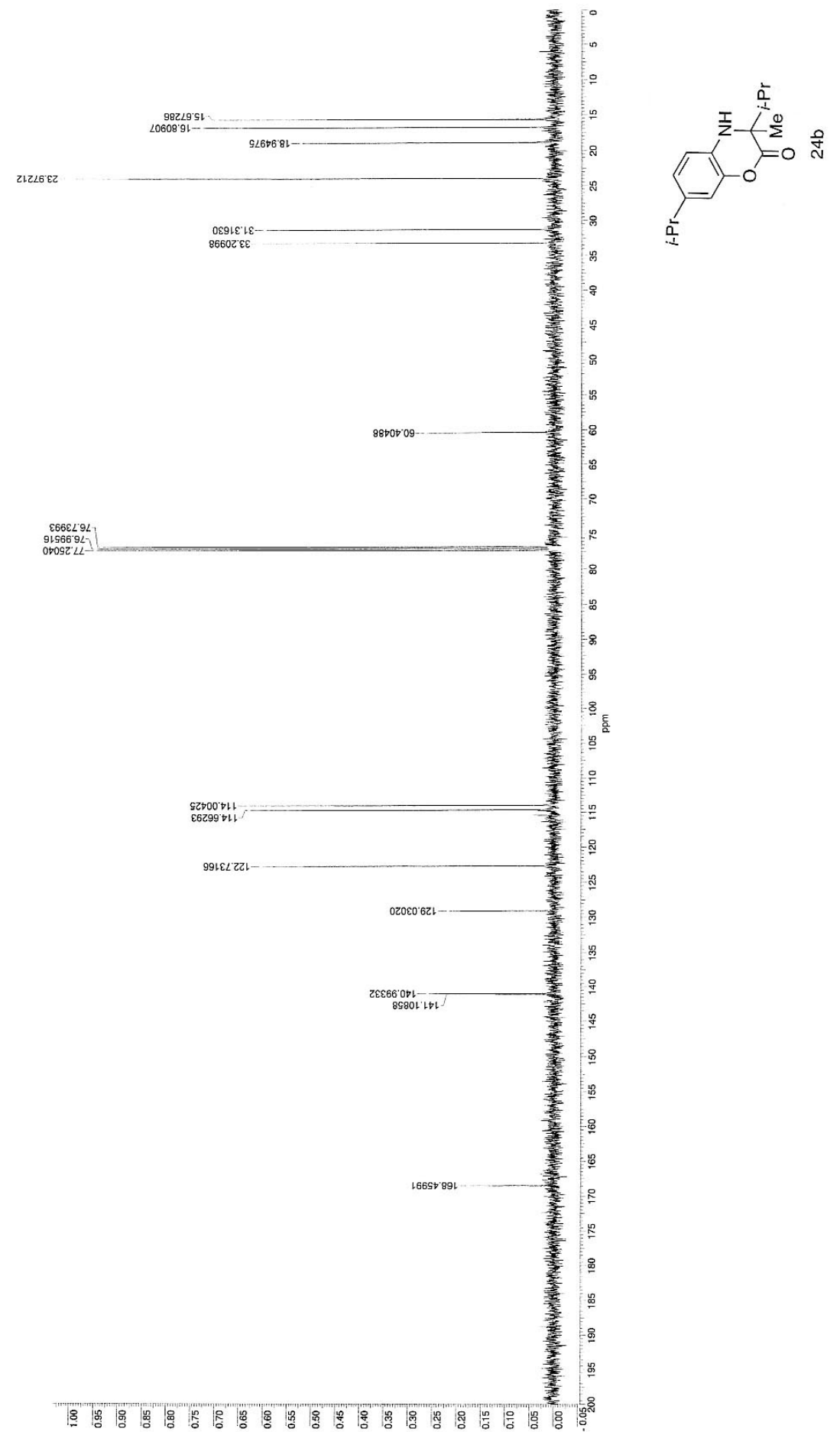

S59 


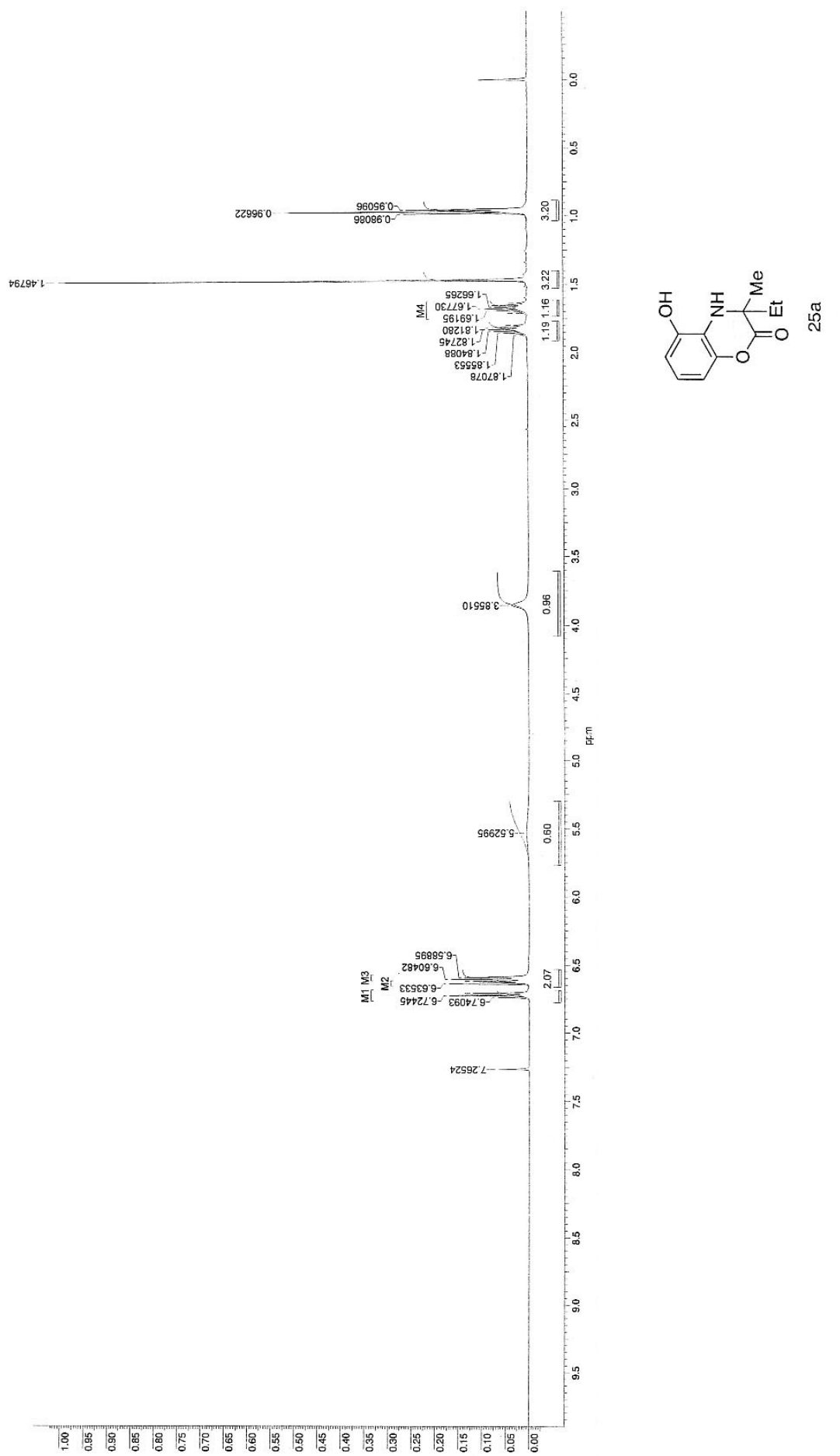




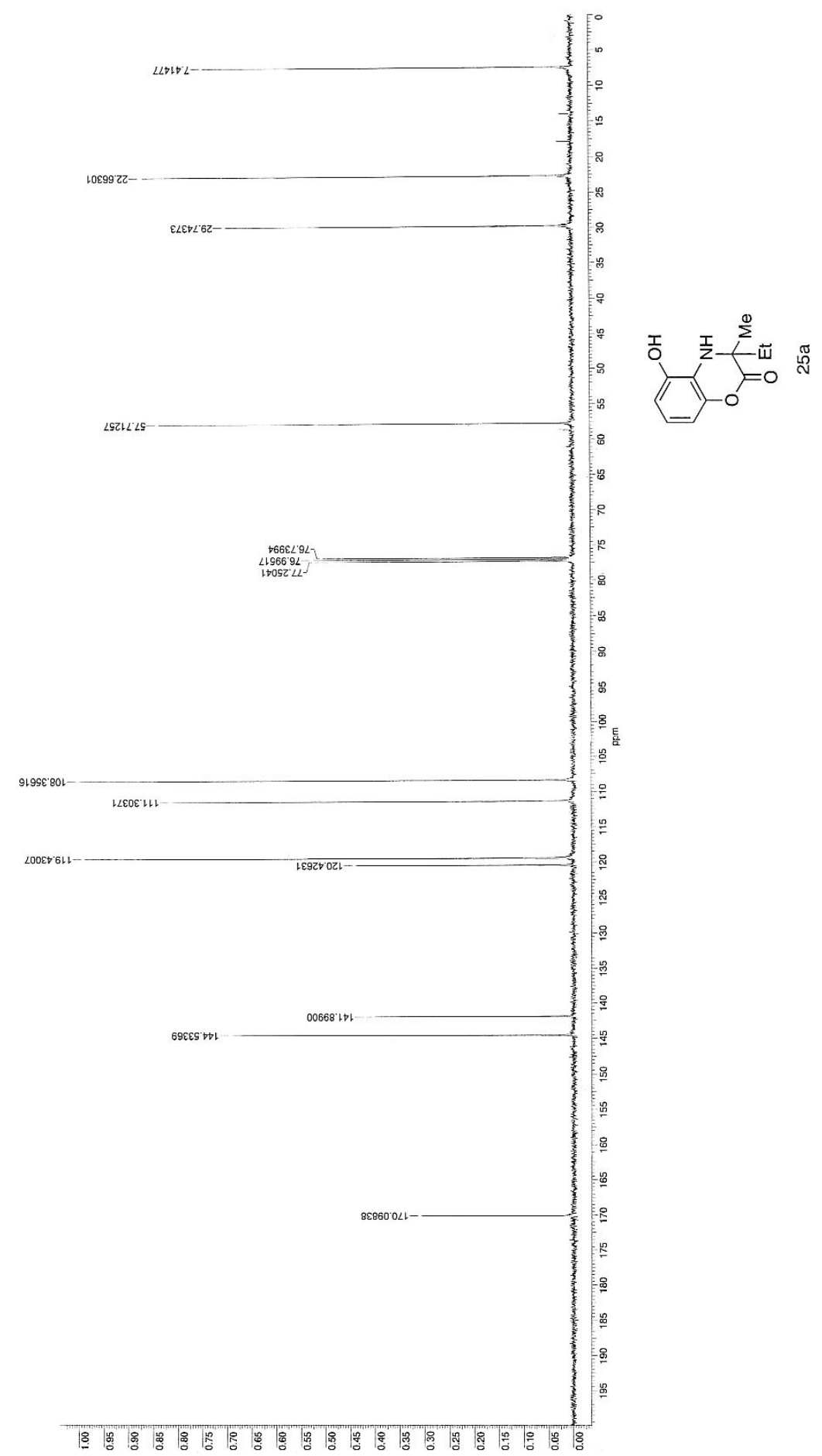




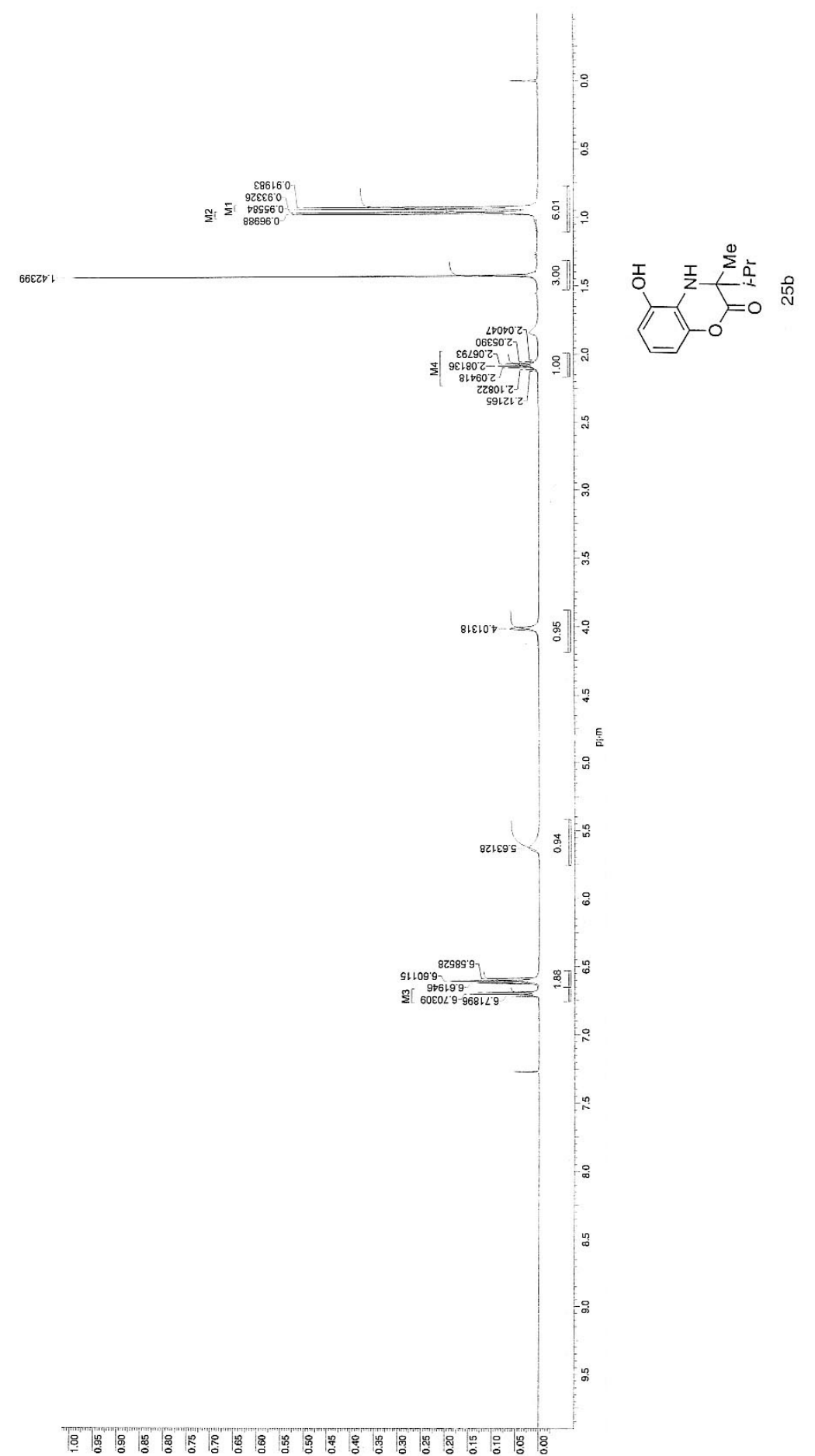

S62 


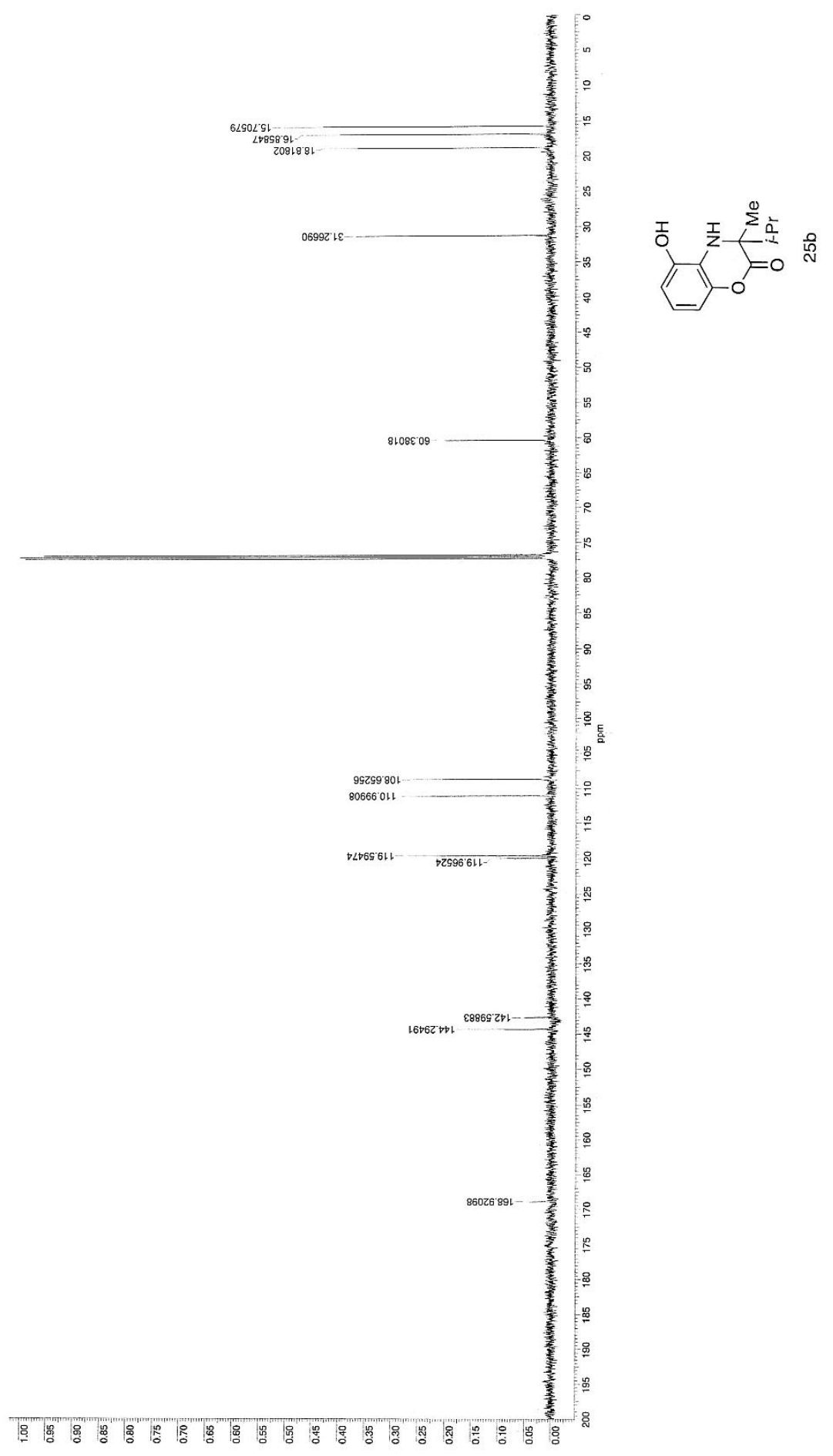




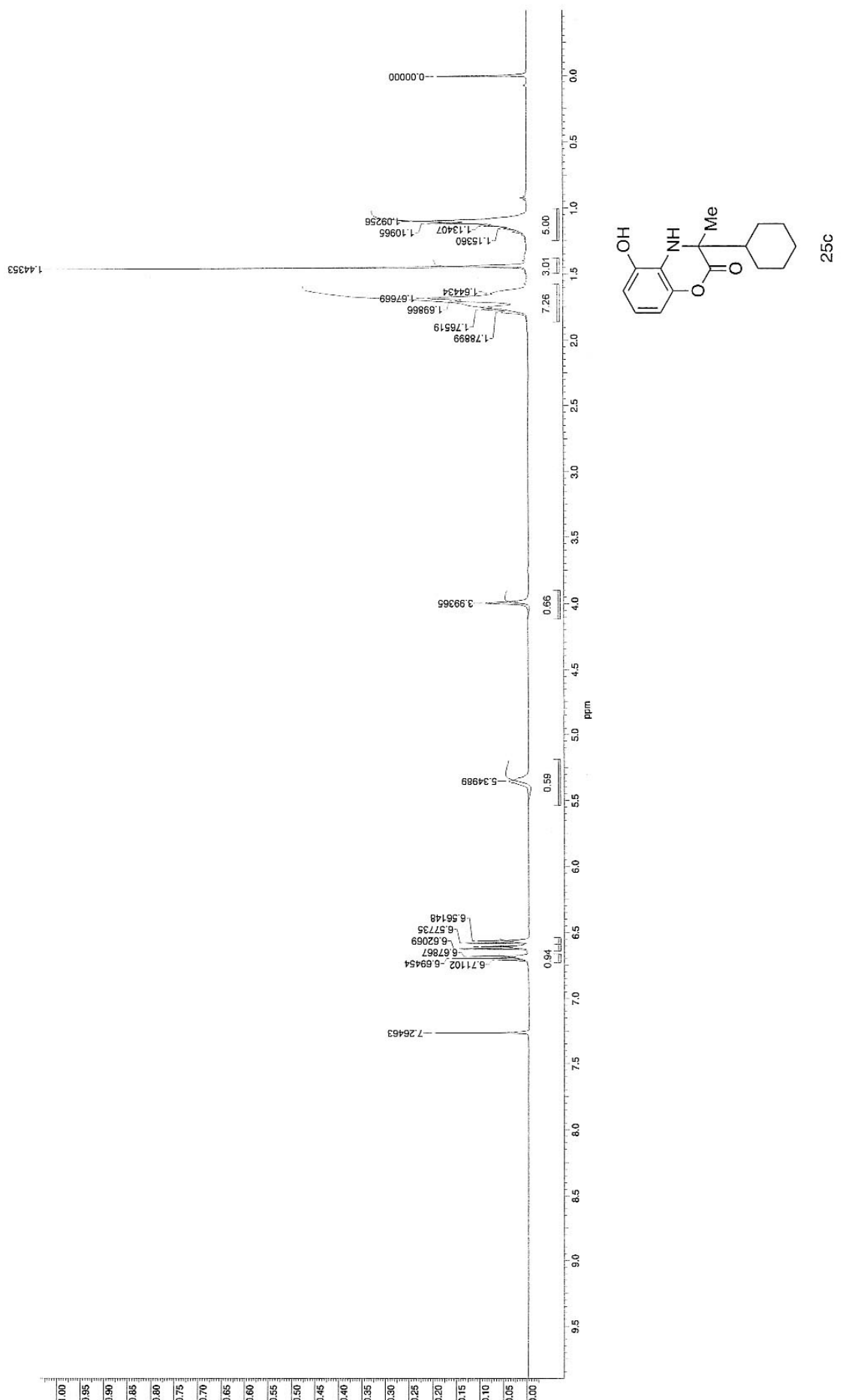

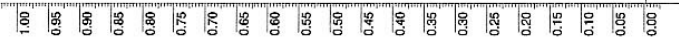




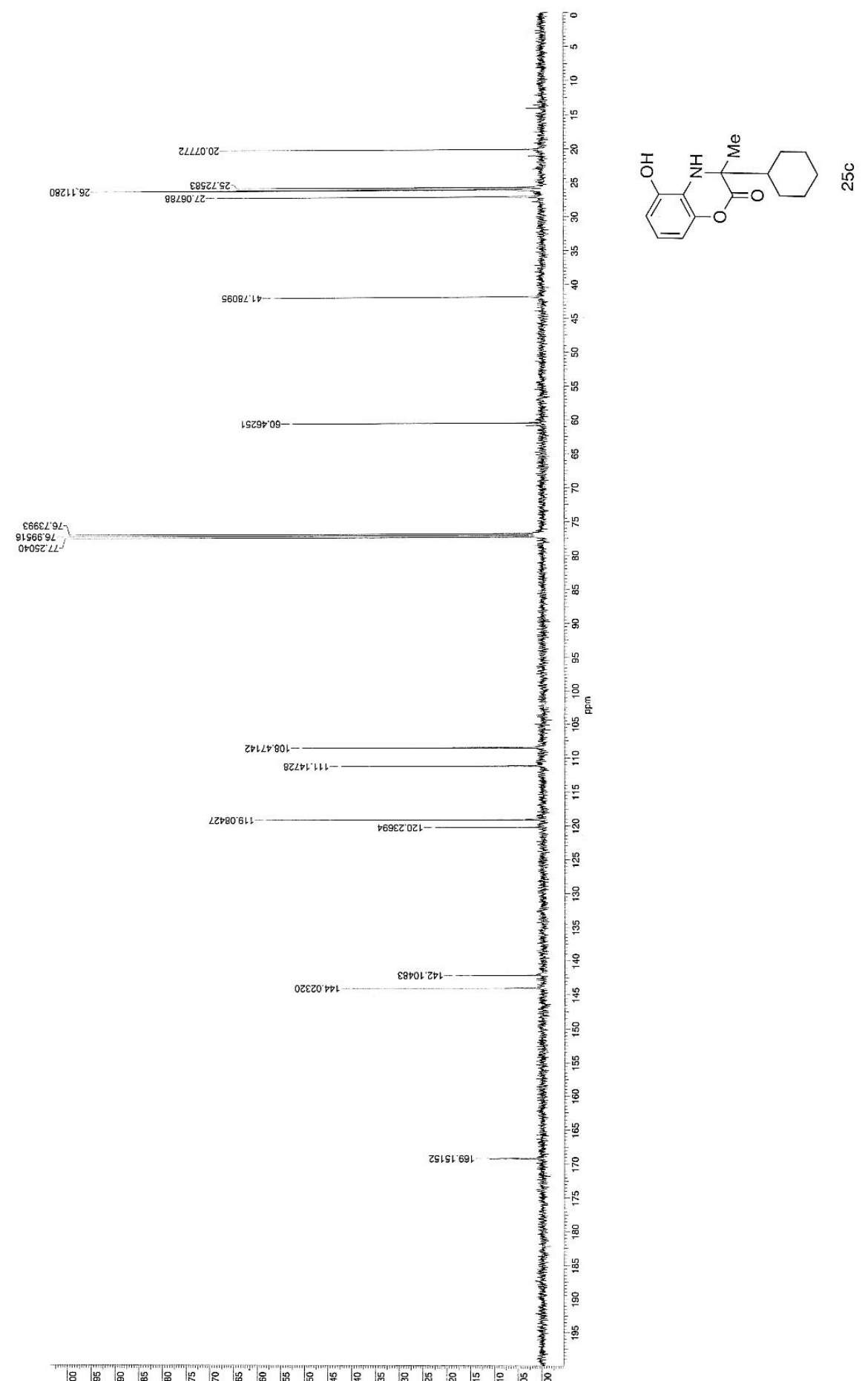

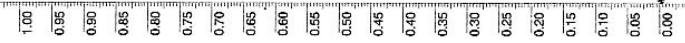




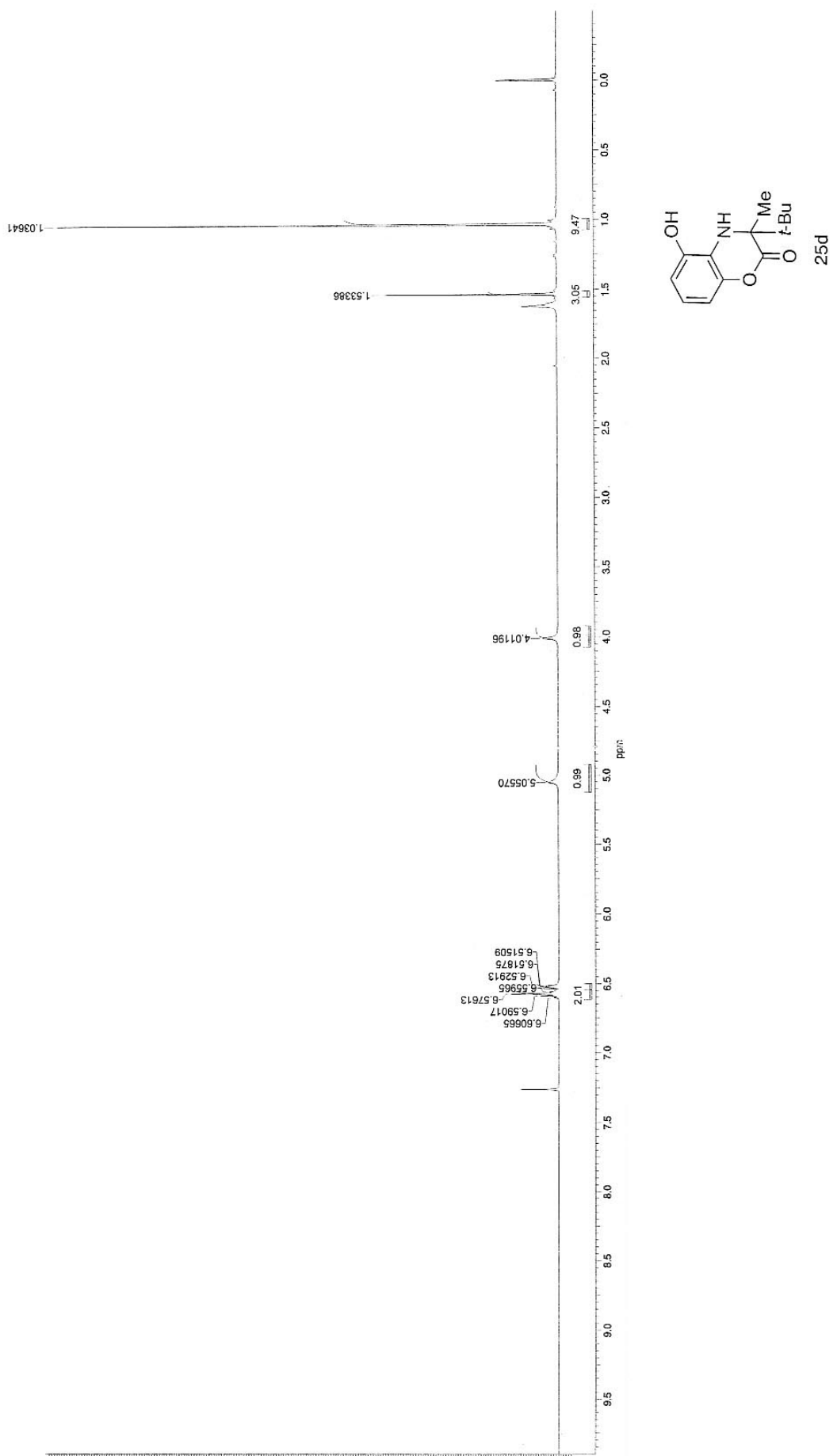

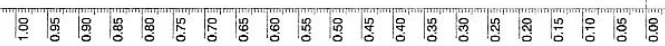




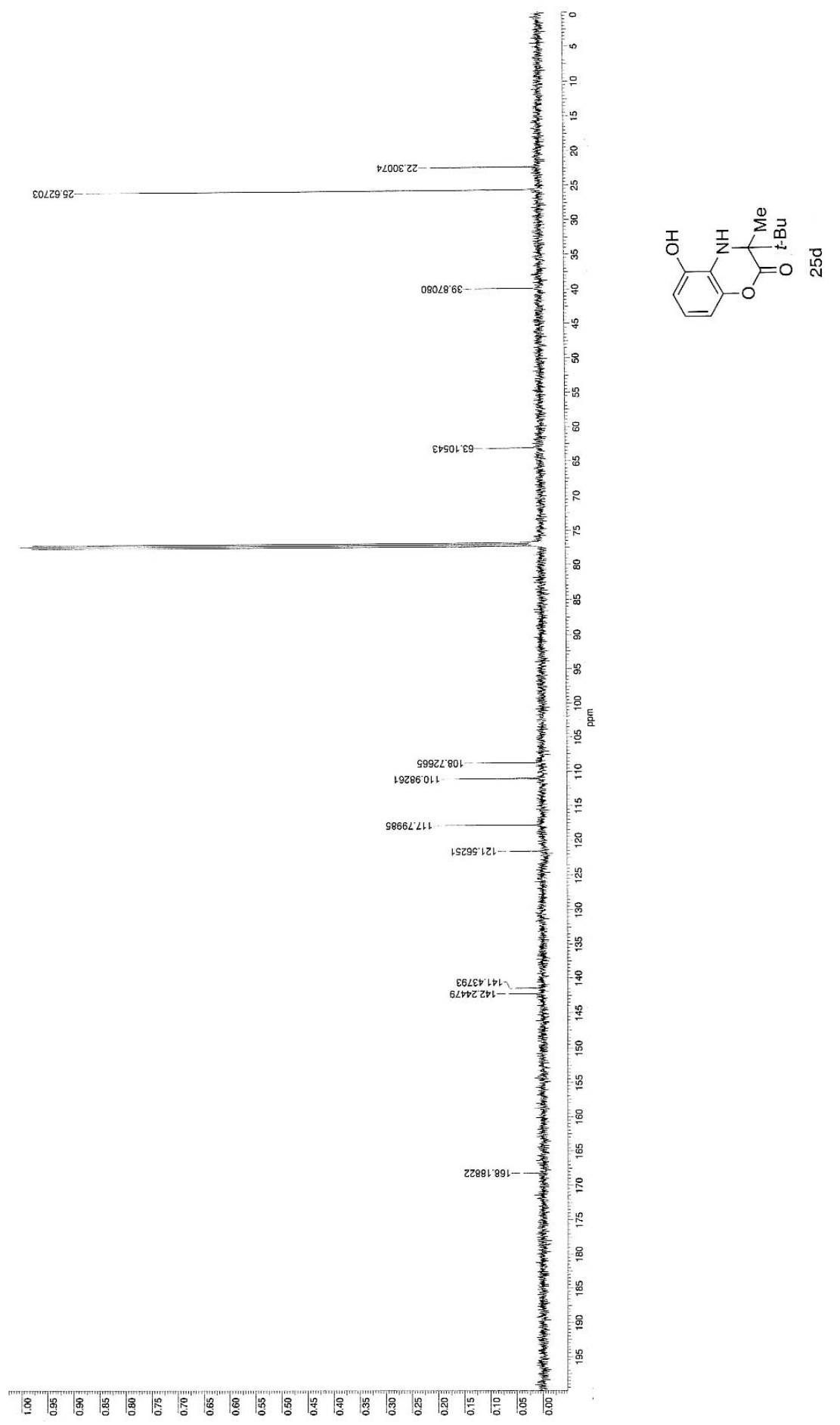




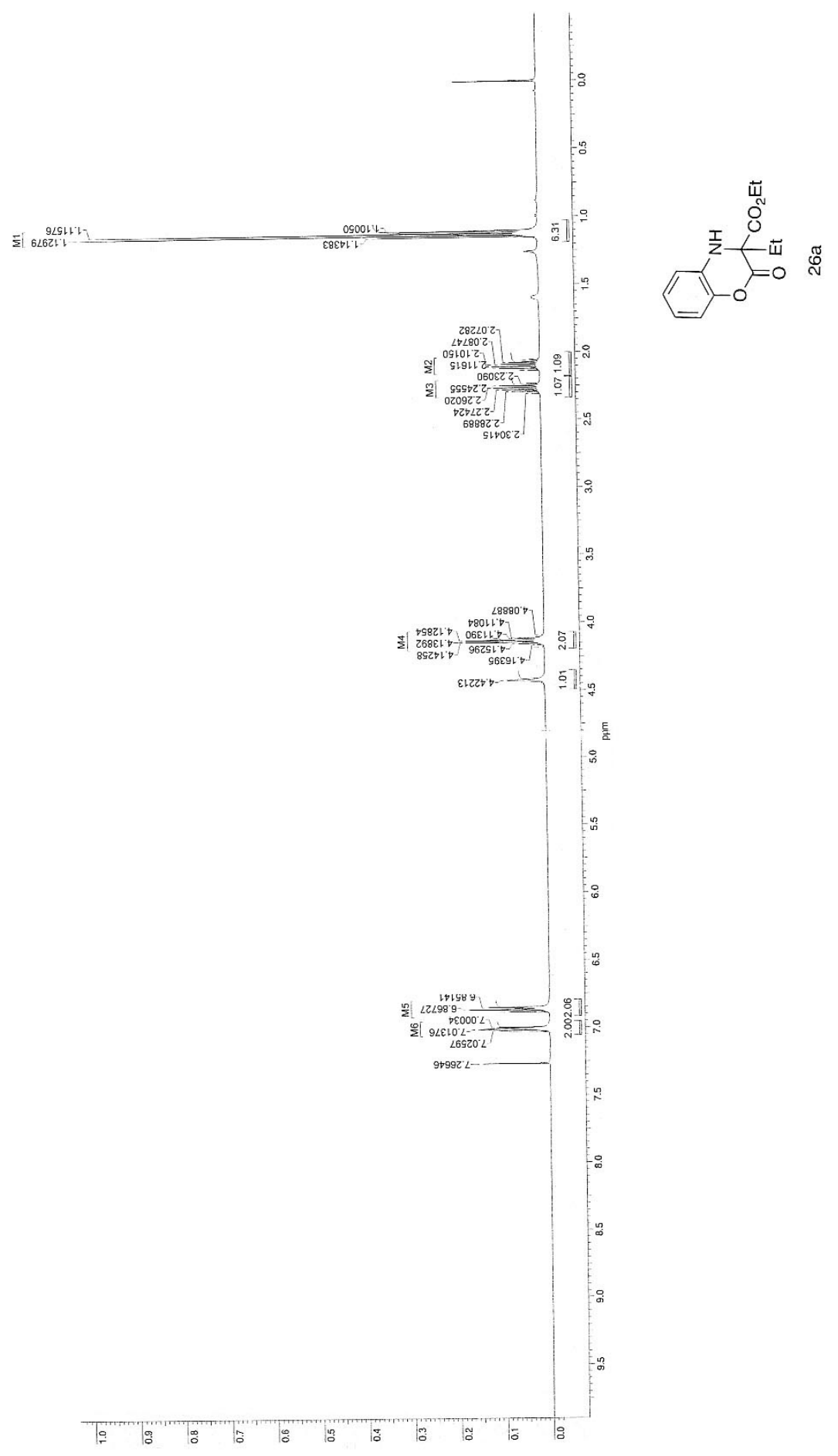



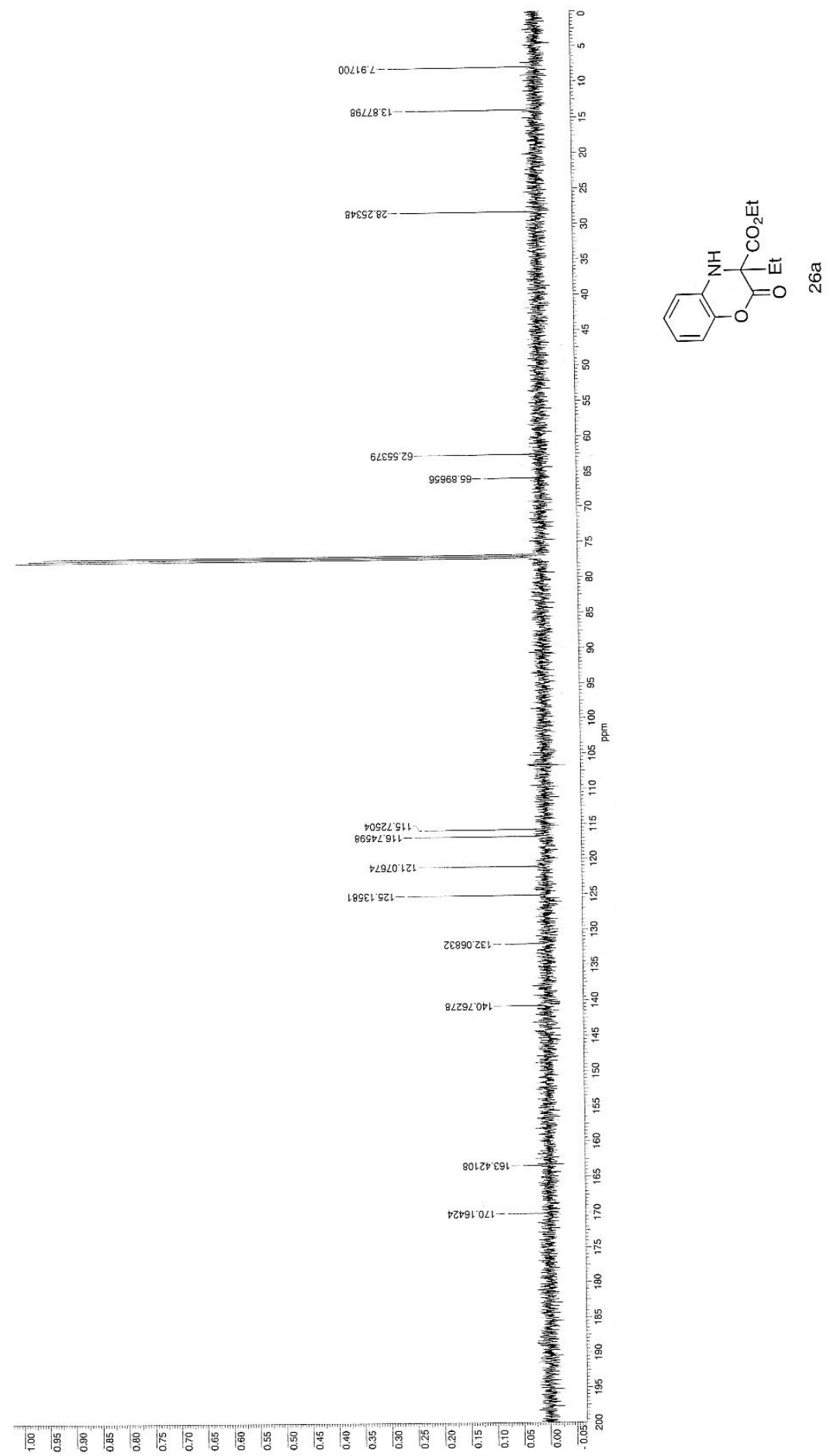

S69 


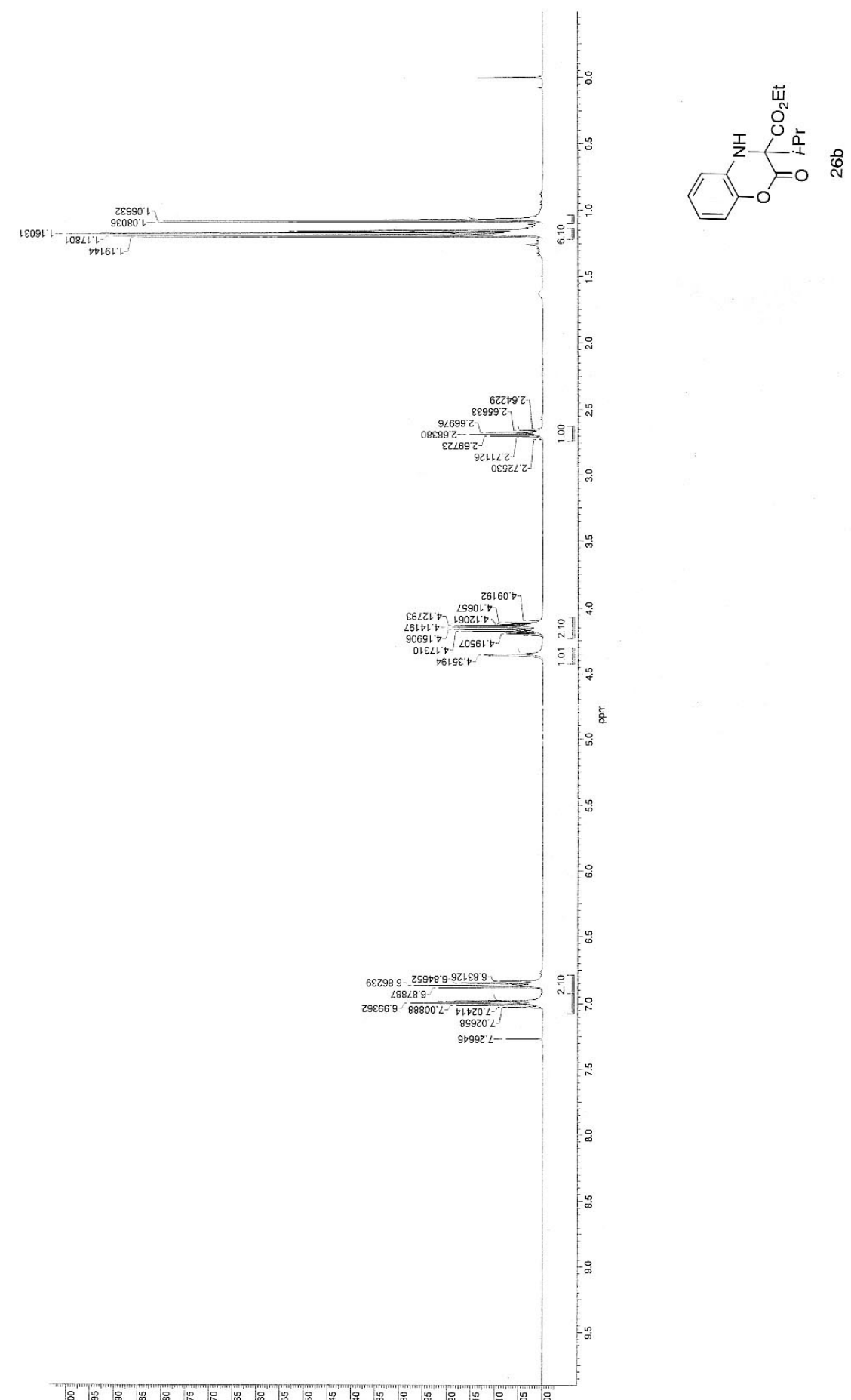

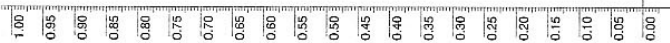




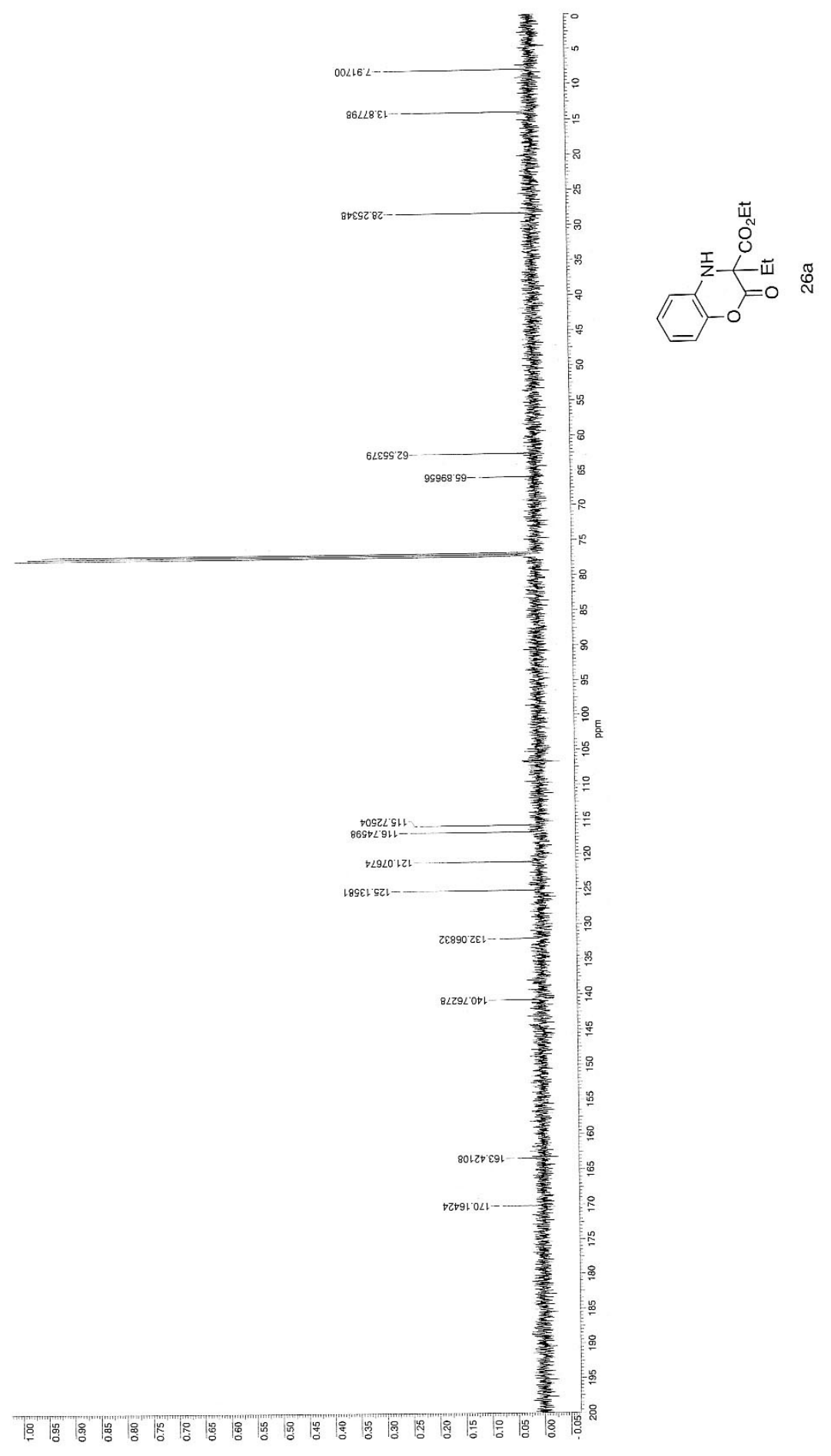

S71 


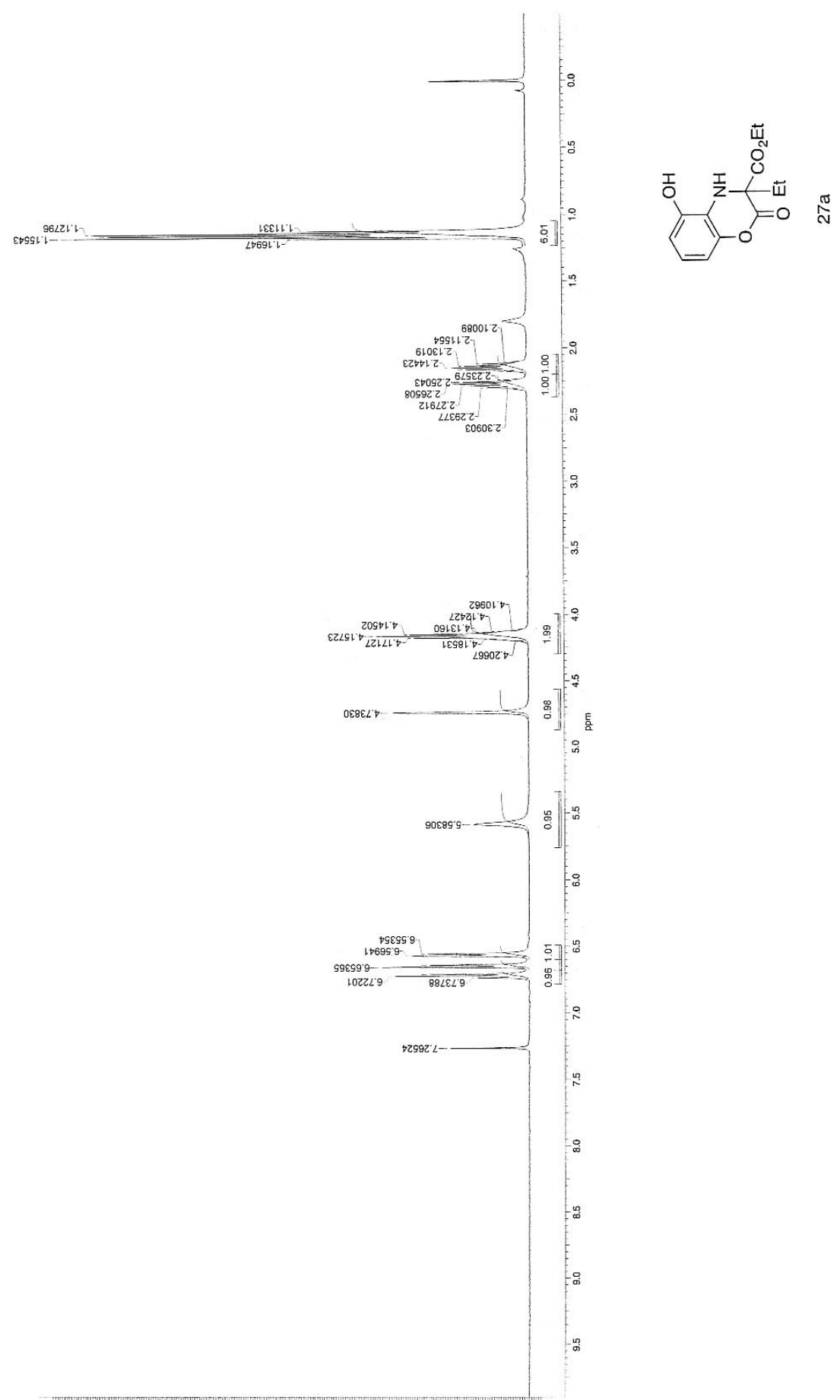

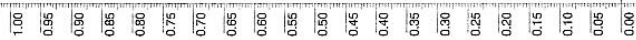




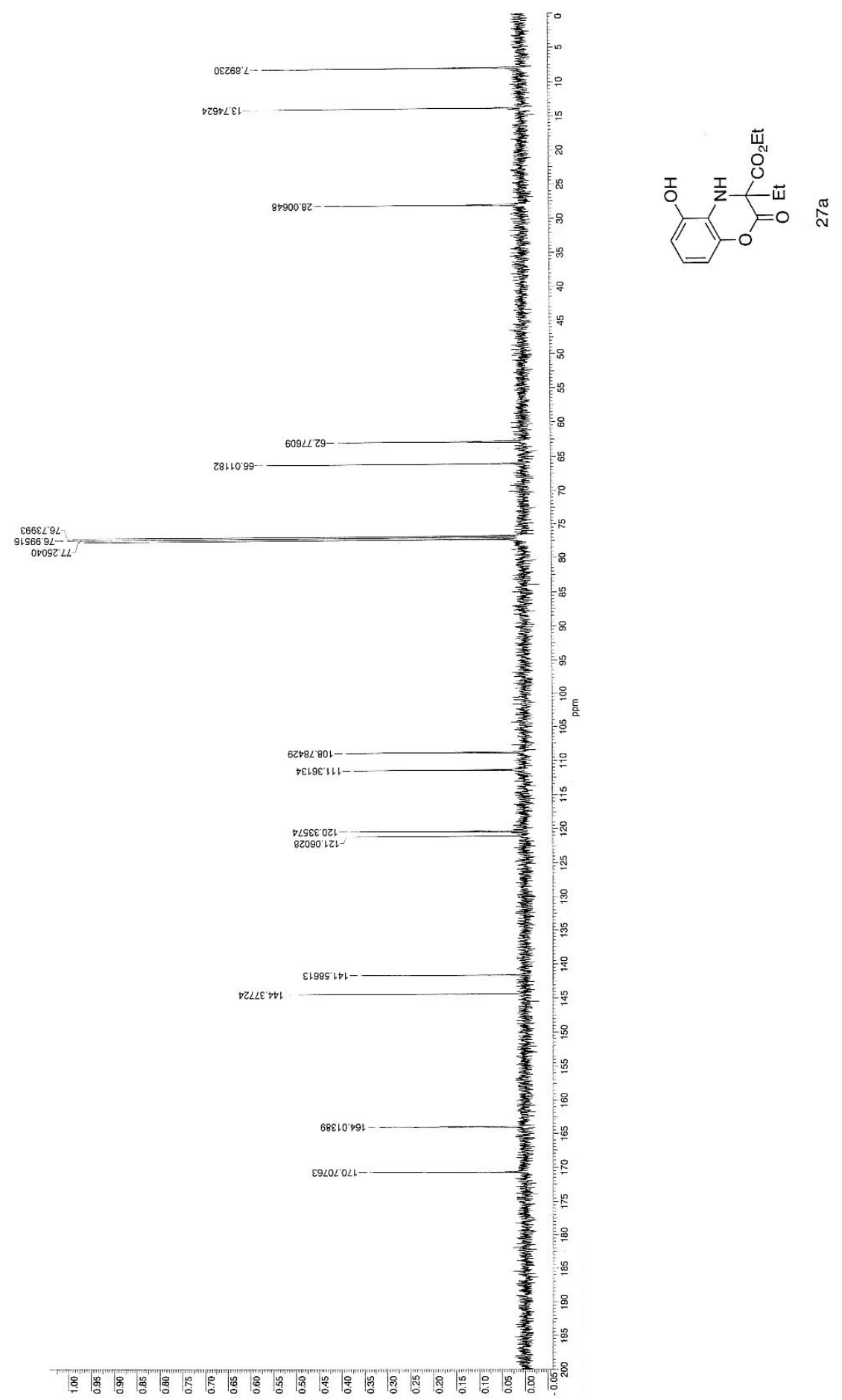




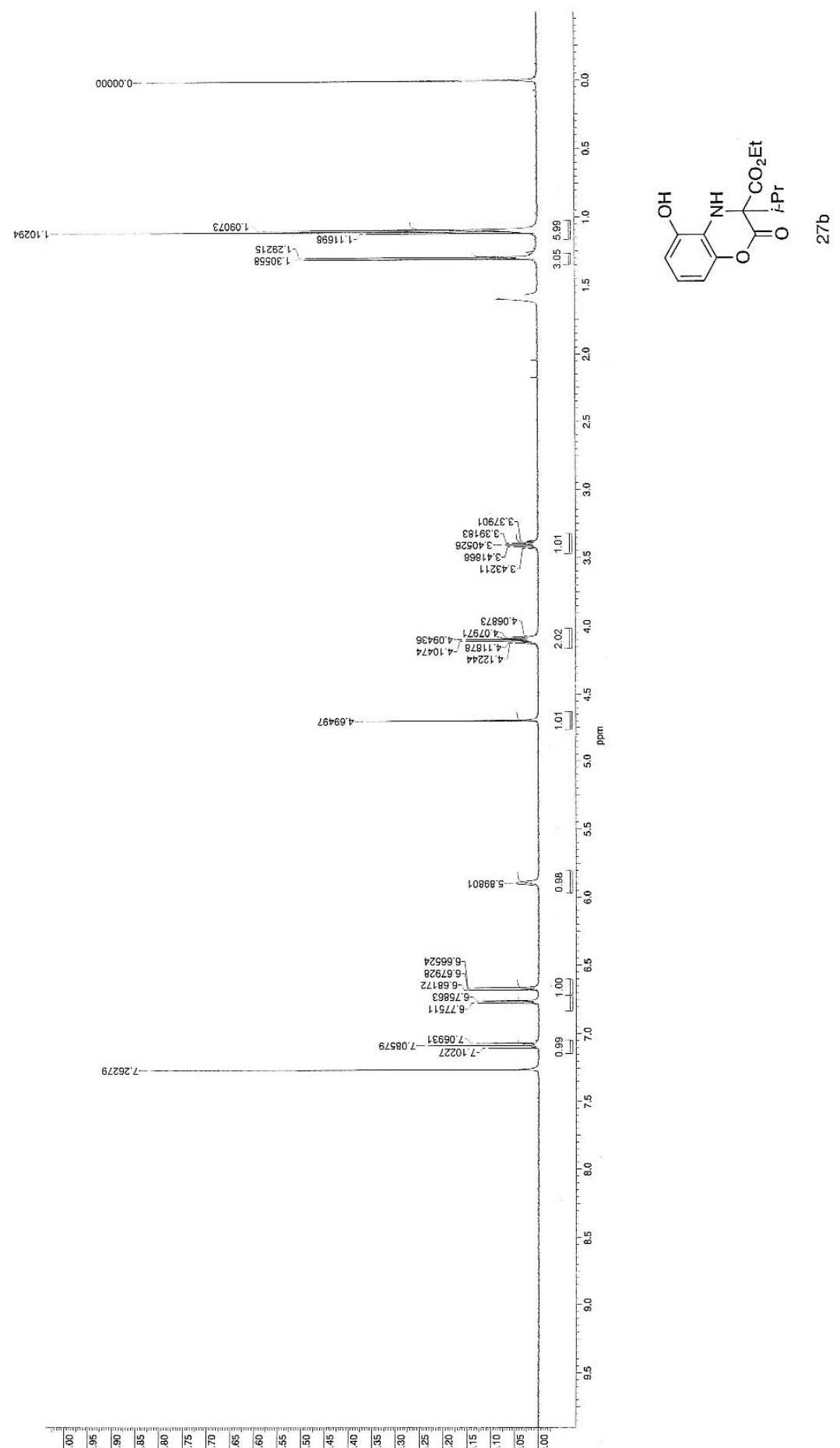

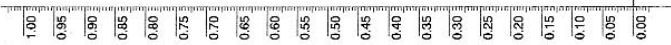




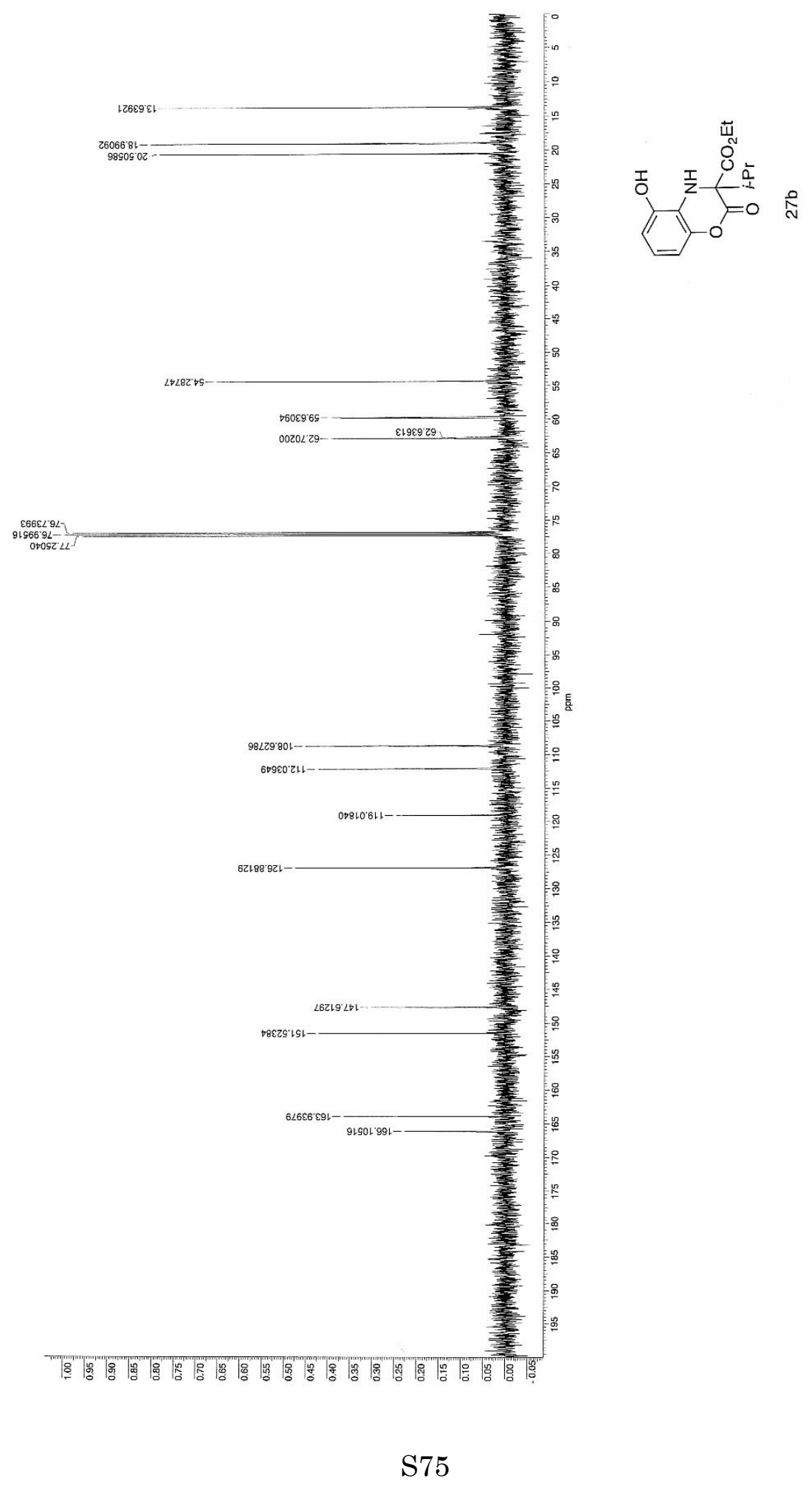




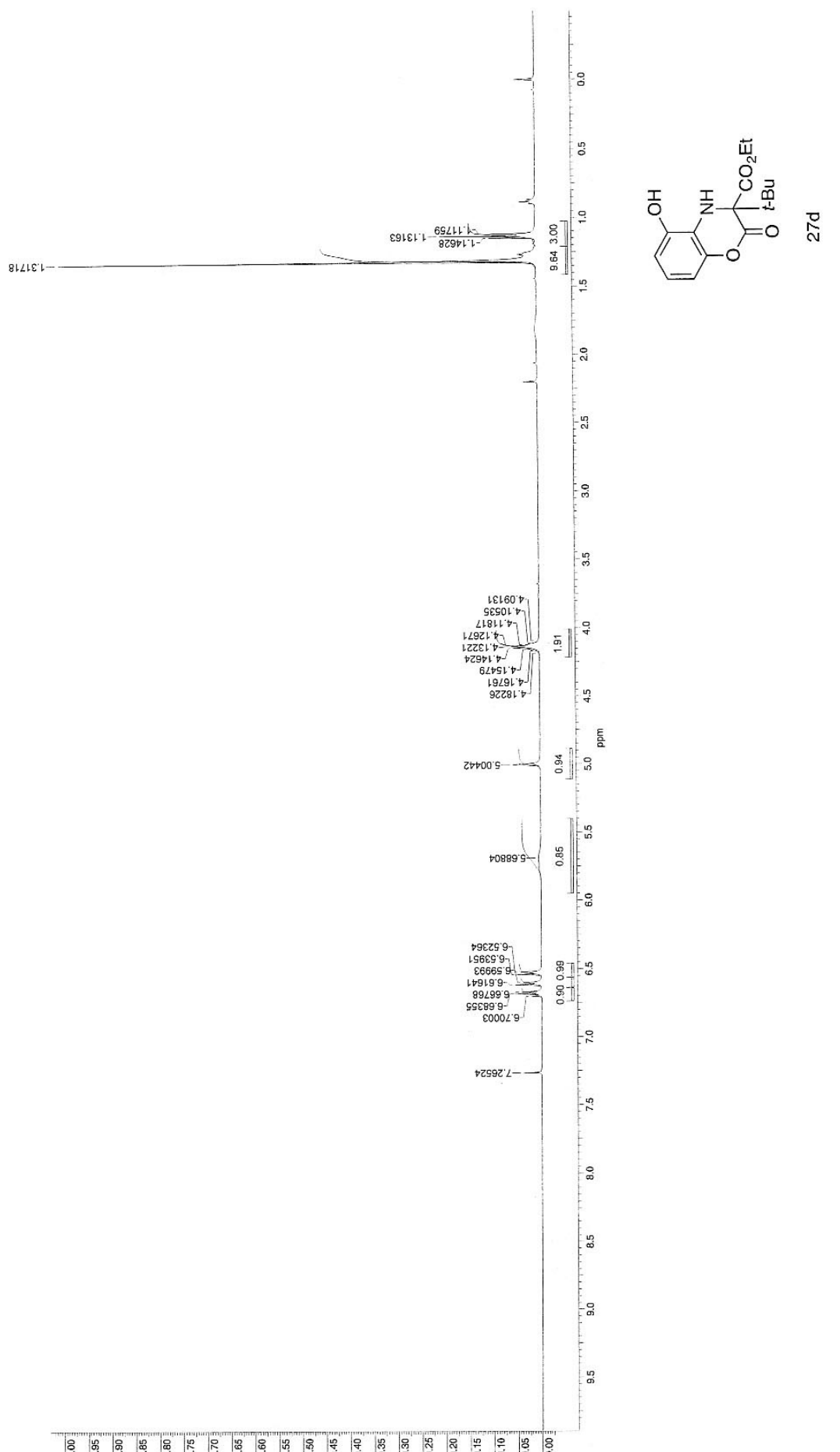

S76 

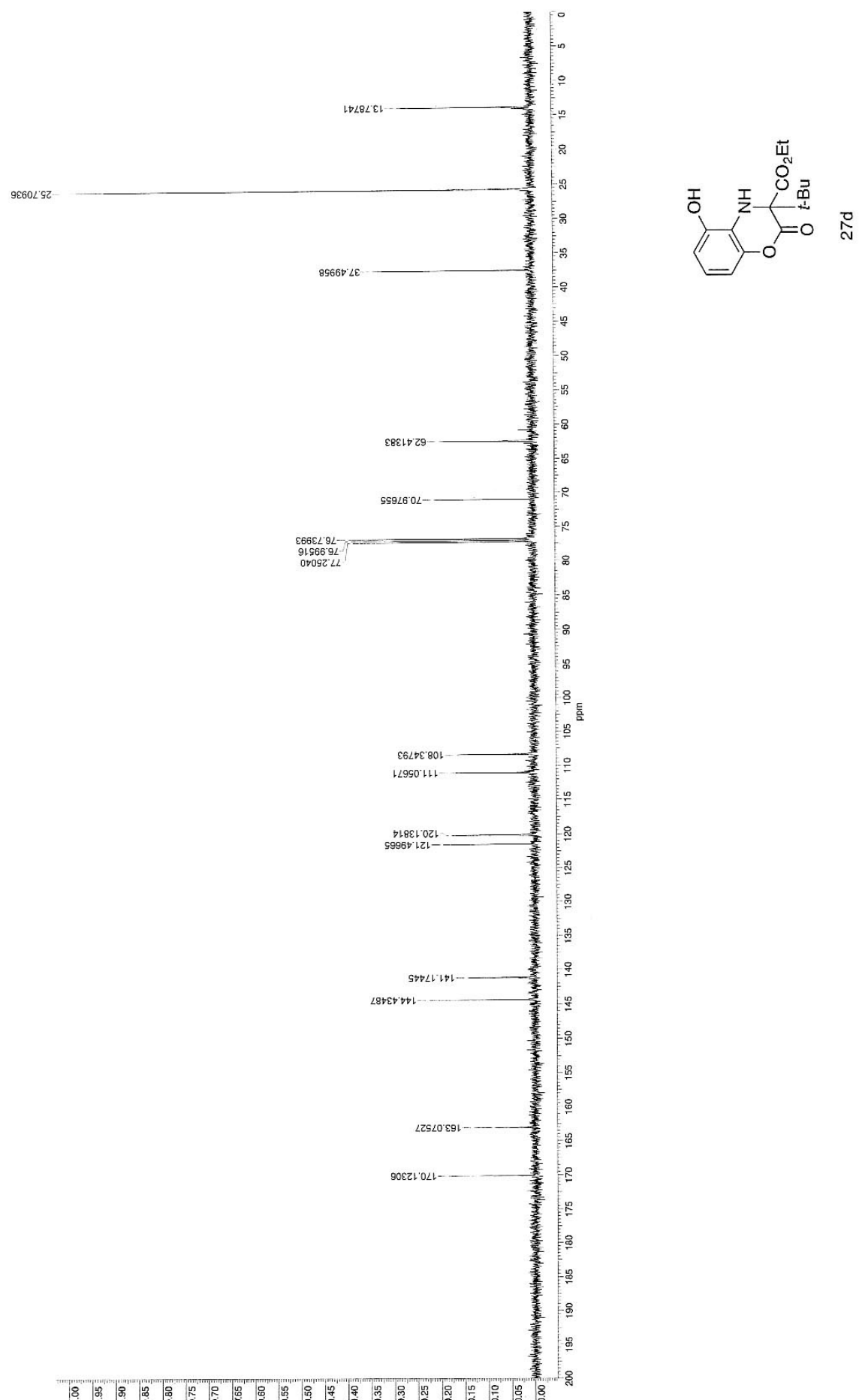


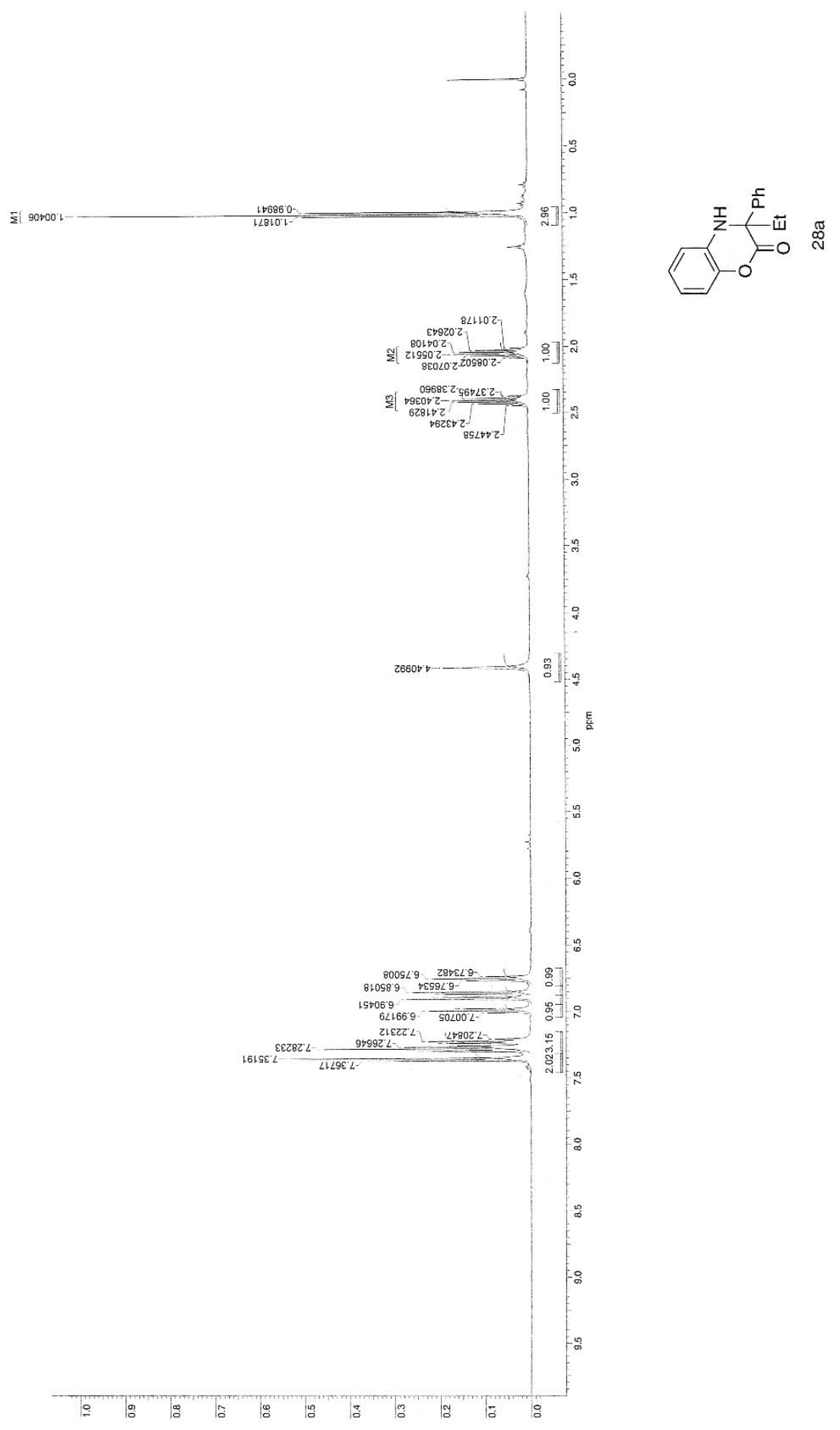

S78 


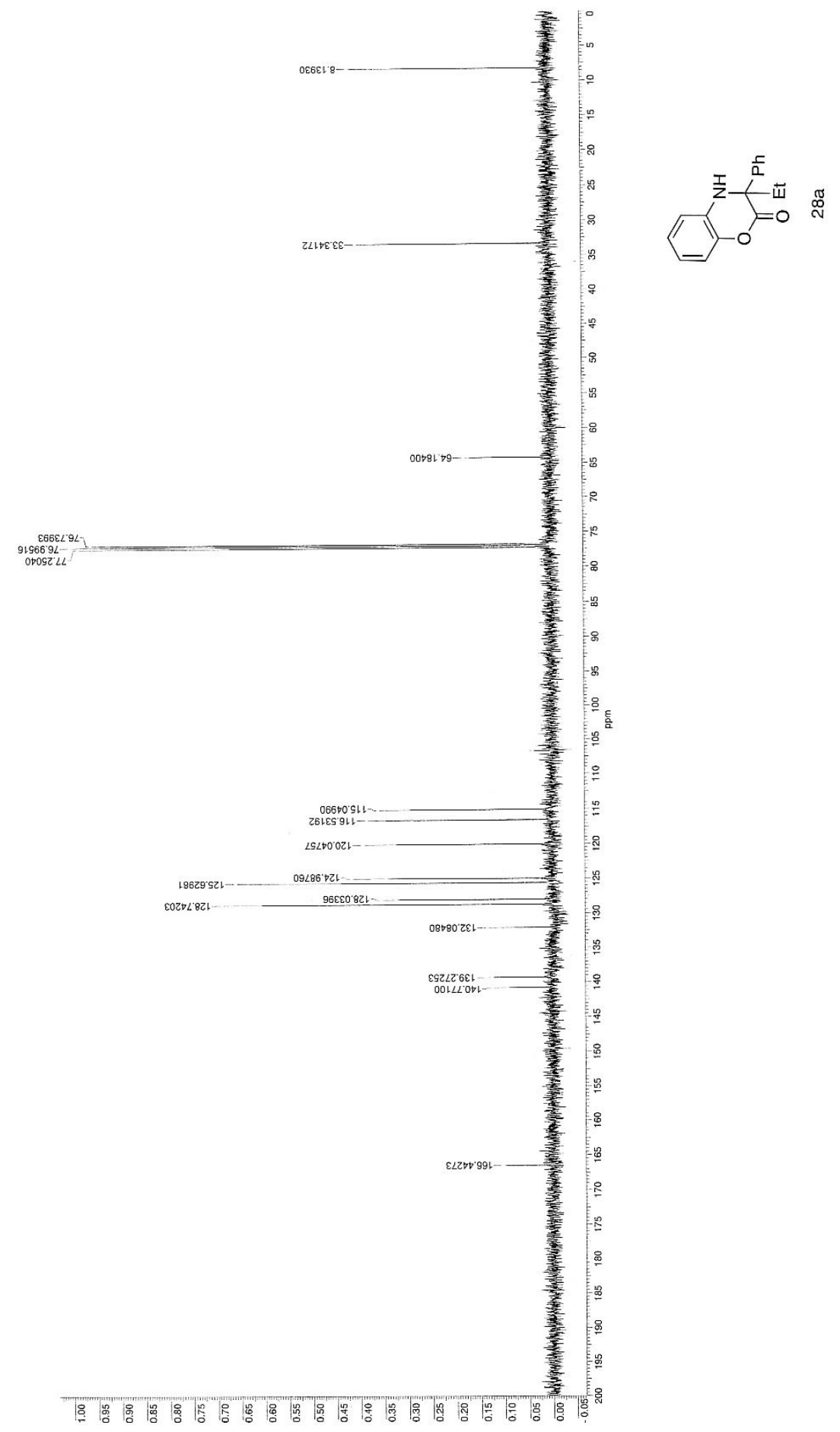

S79 


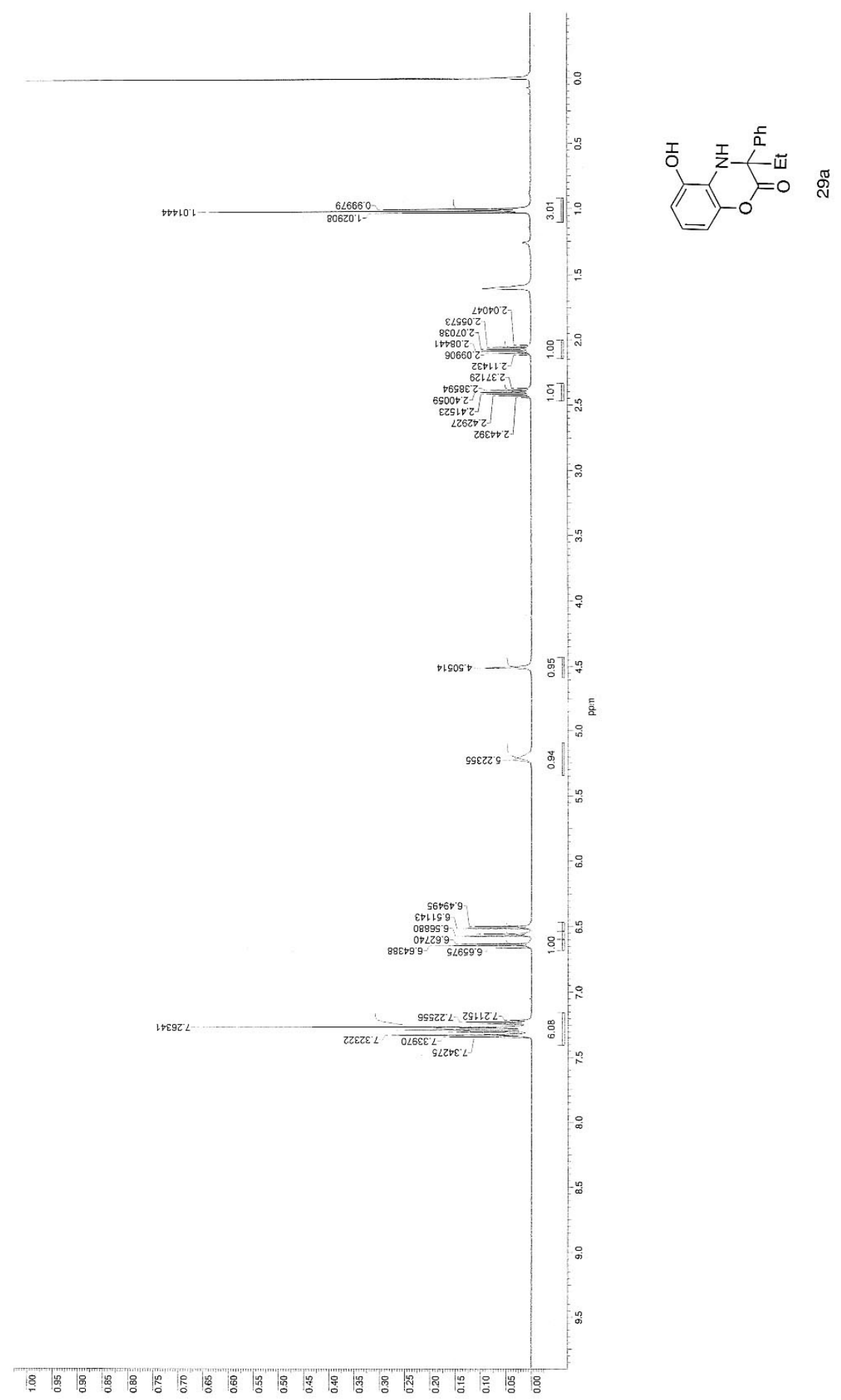

S80 


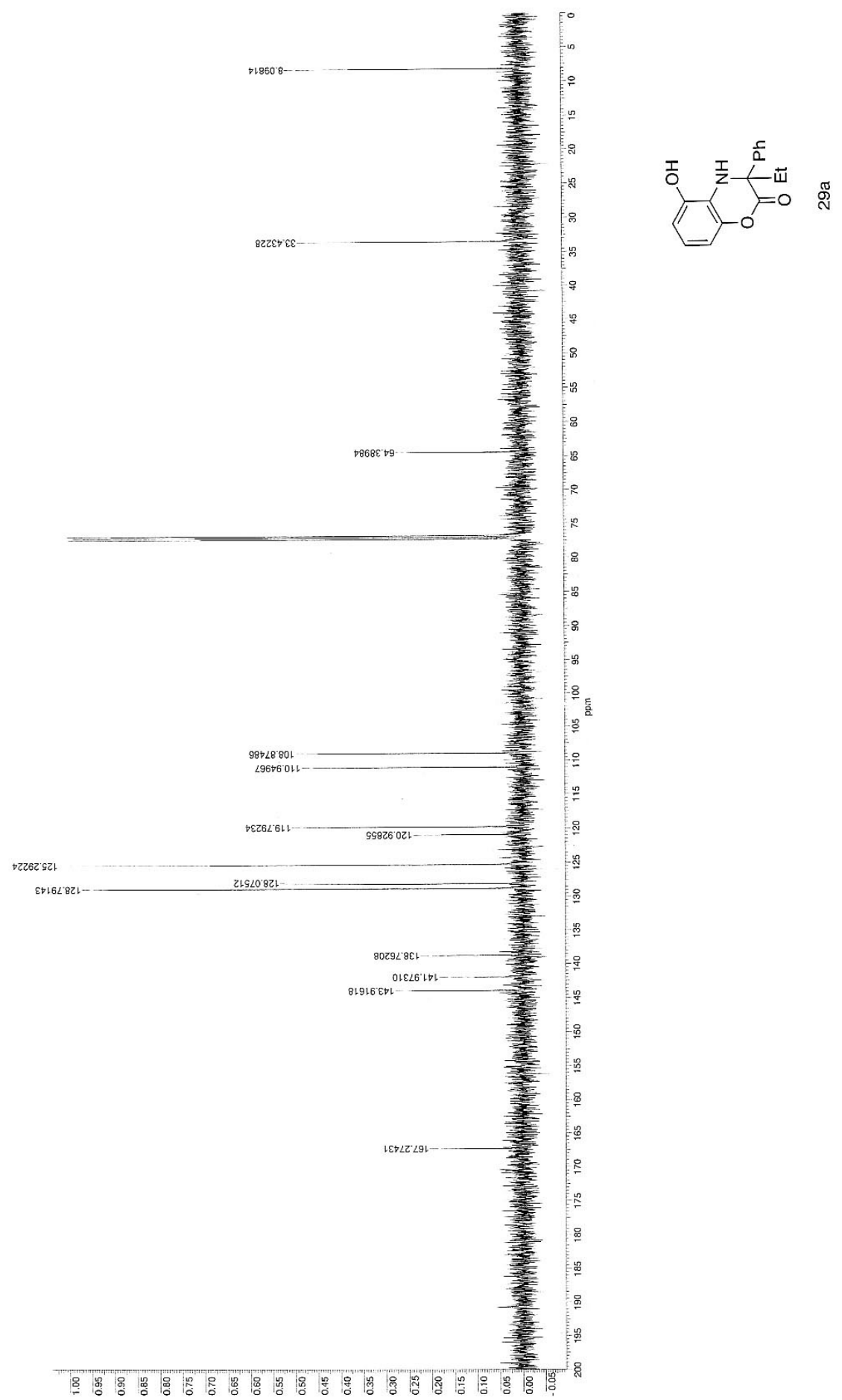

S81 


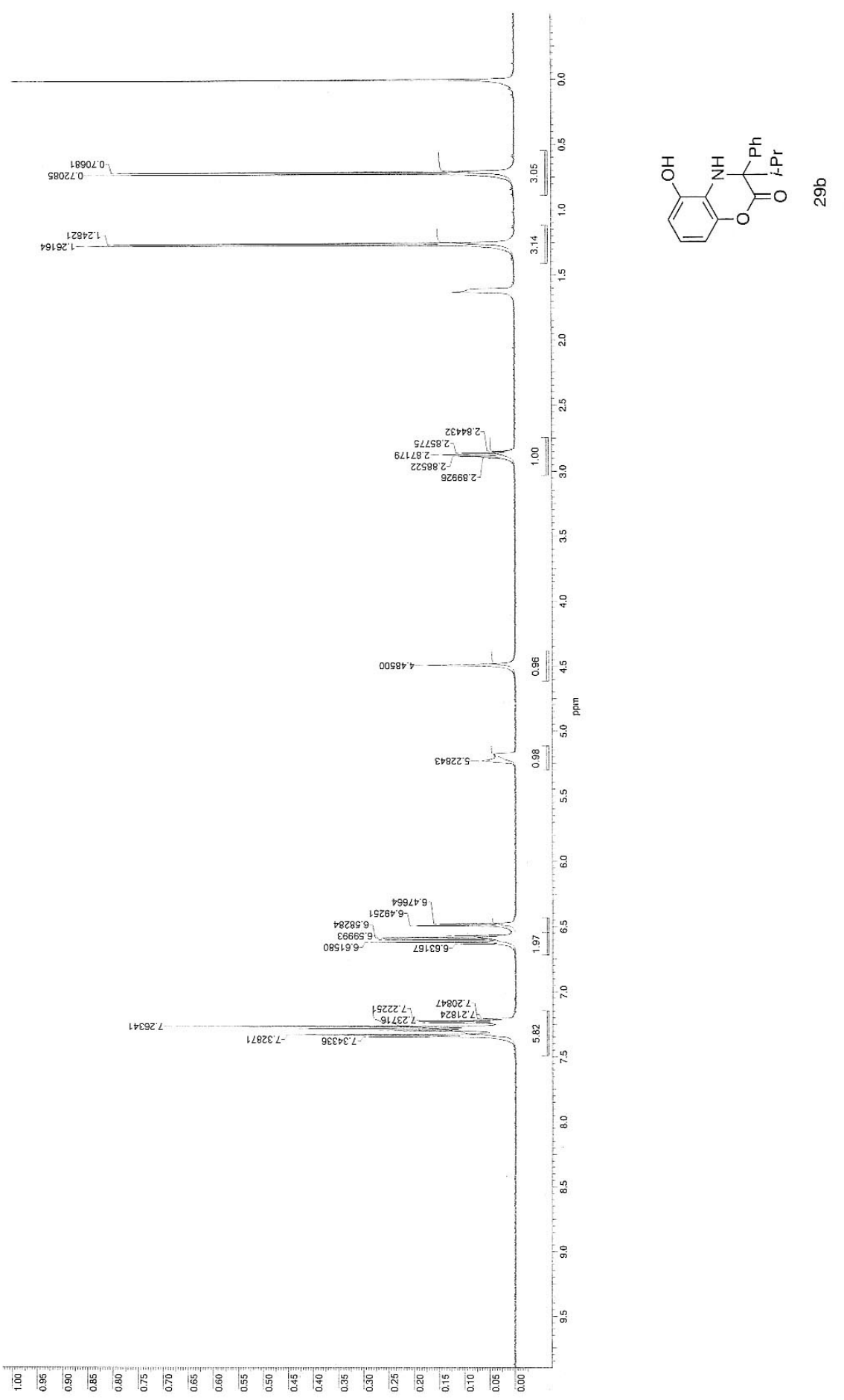

S82 


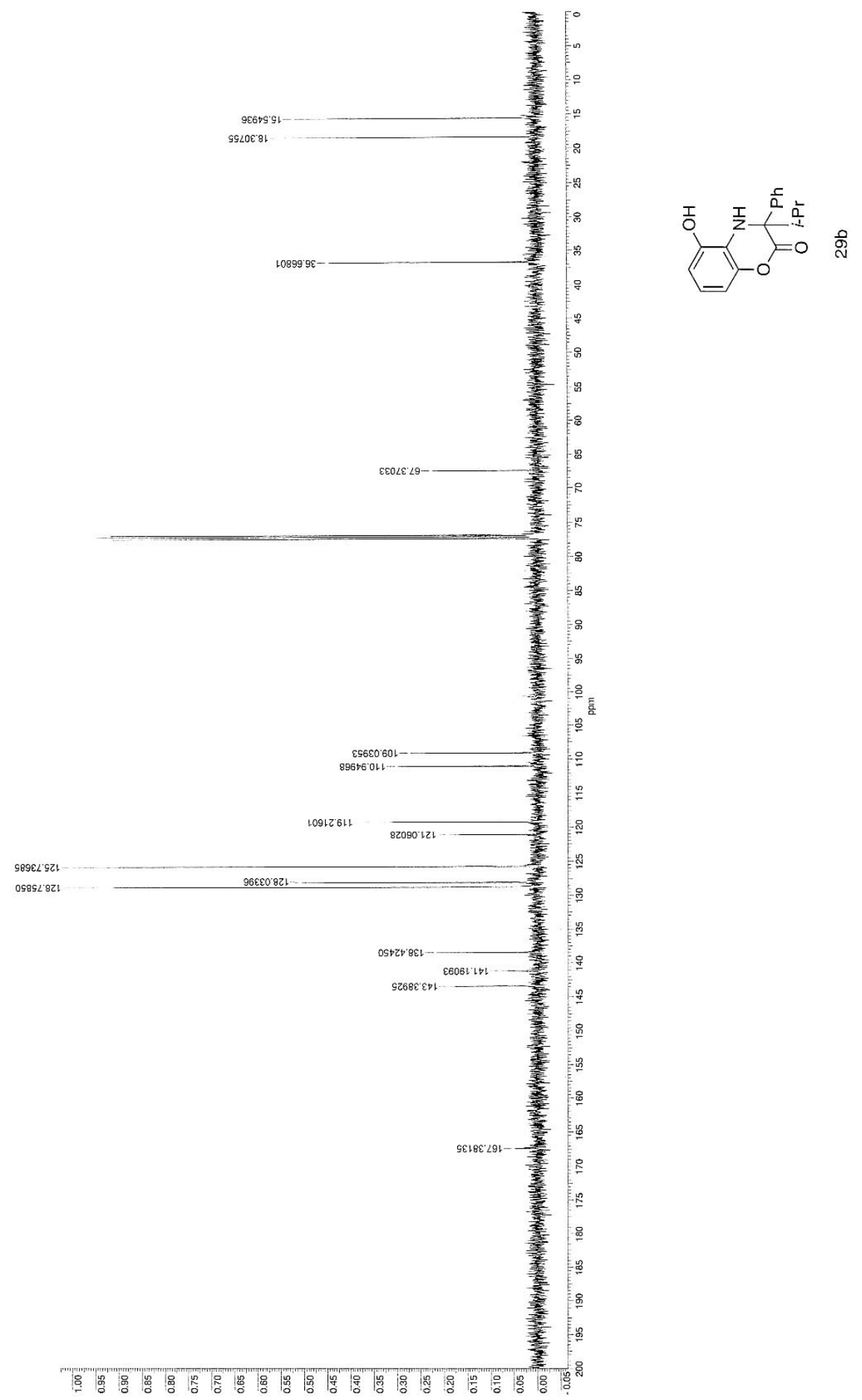

S83 


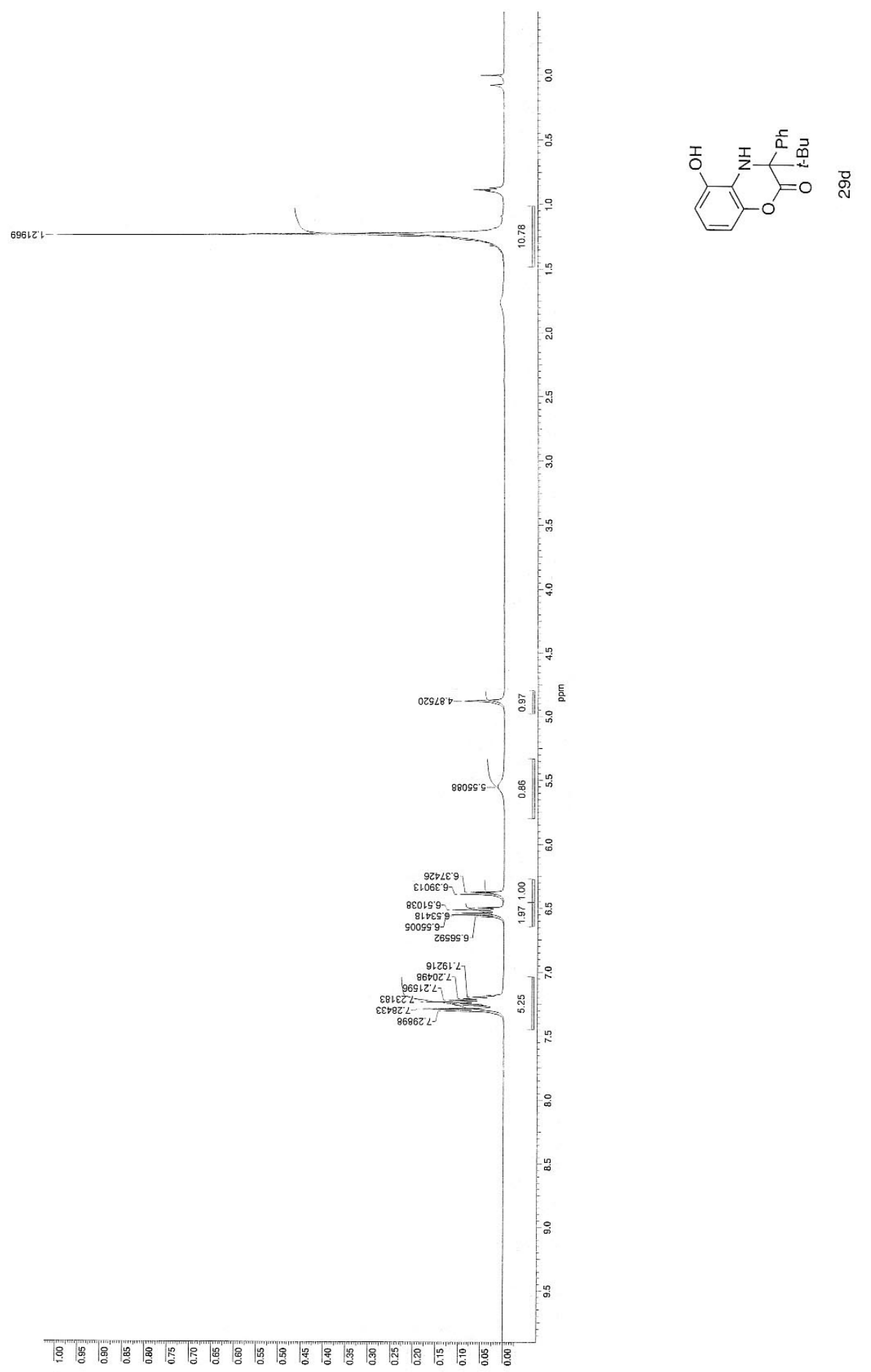

S84 


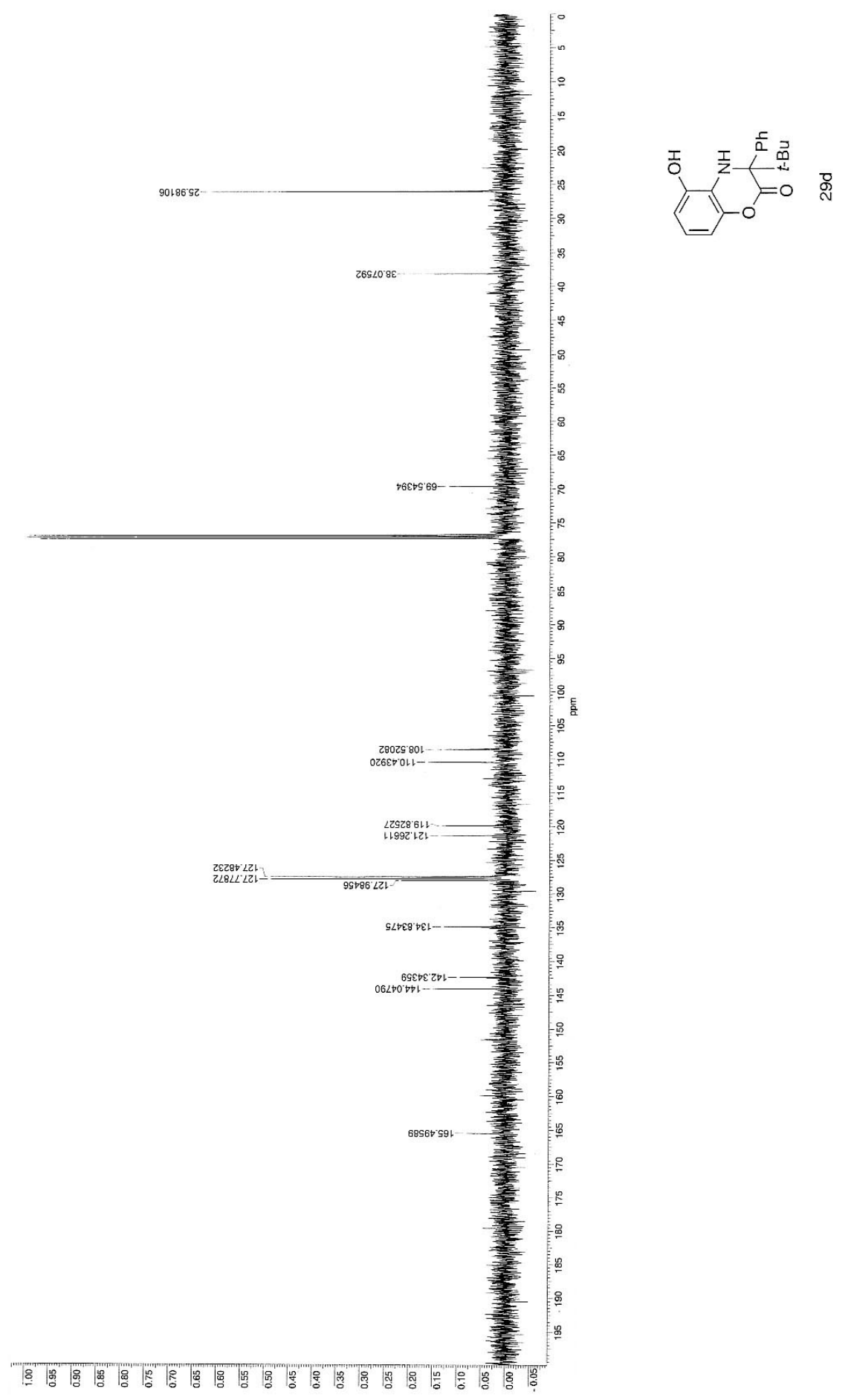

S85 


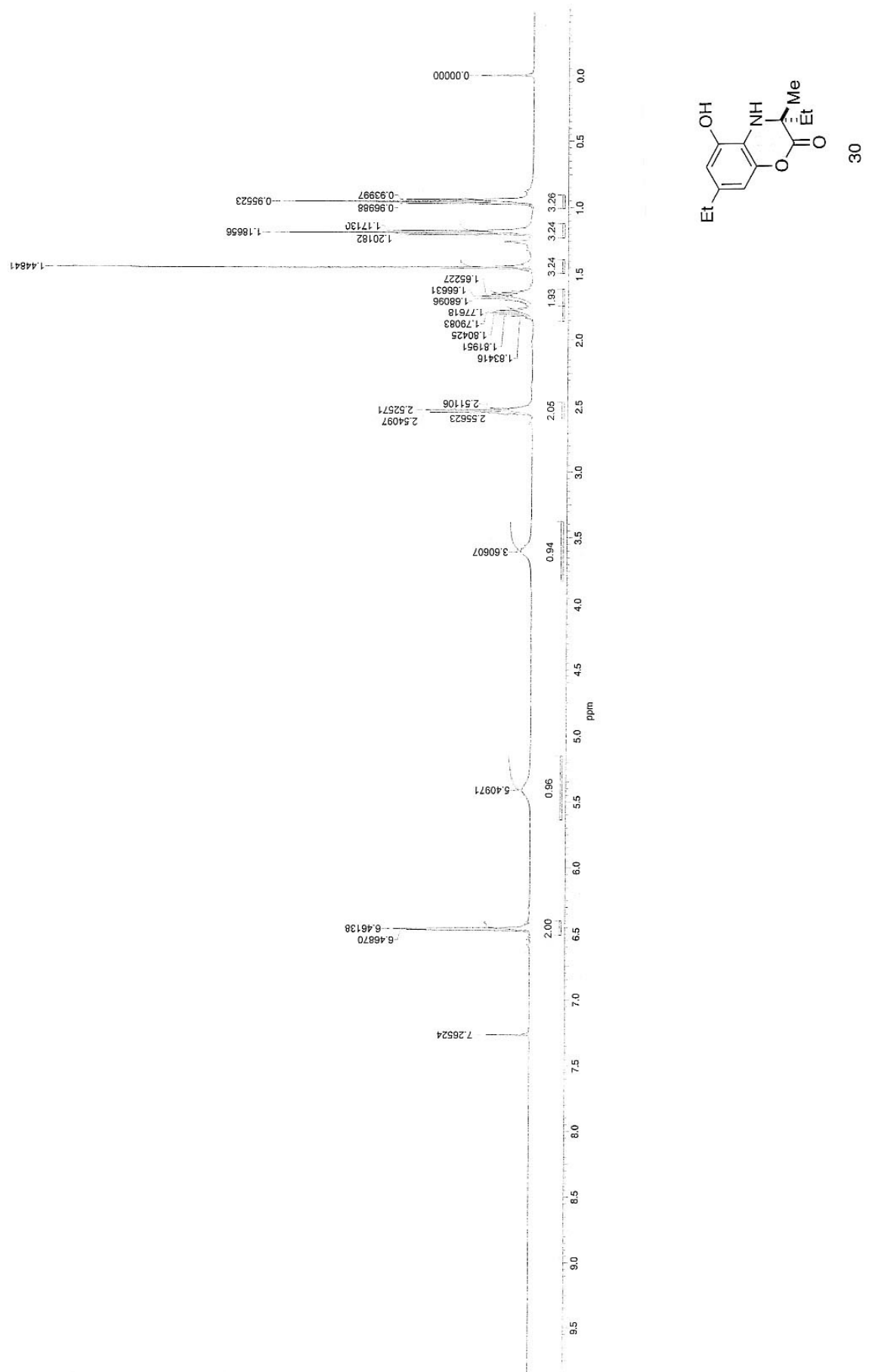

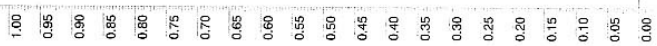

S86 


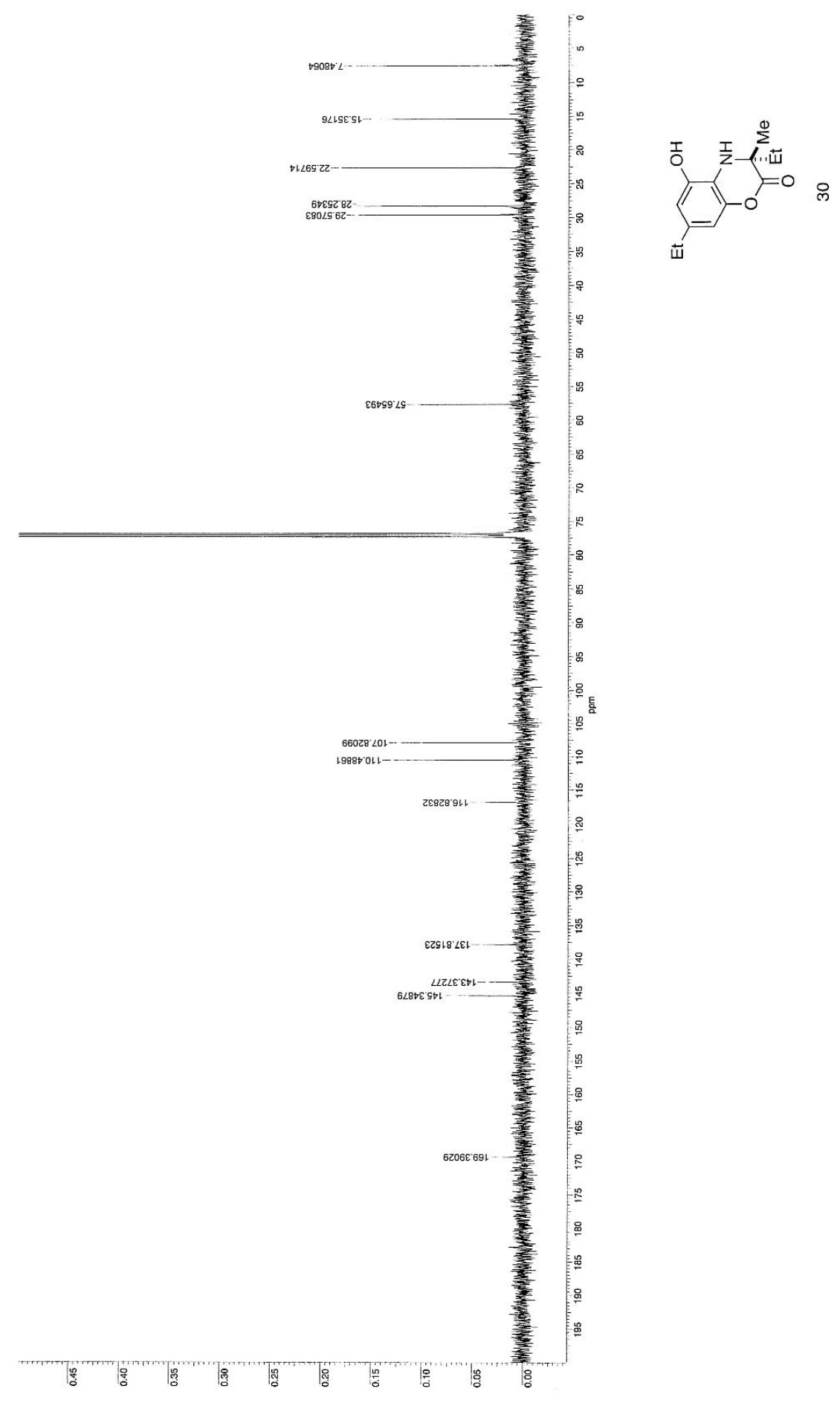

S87 


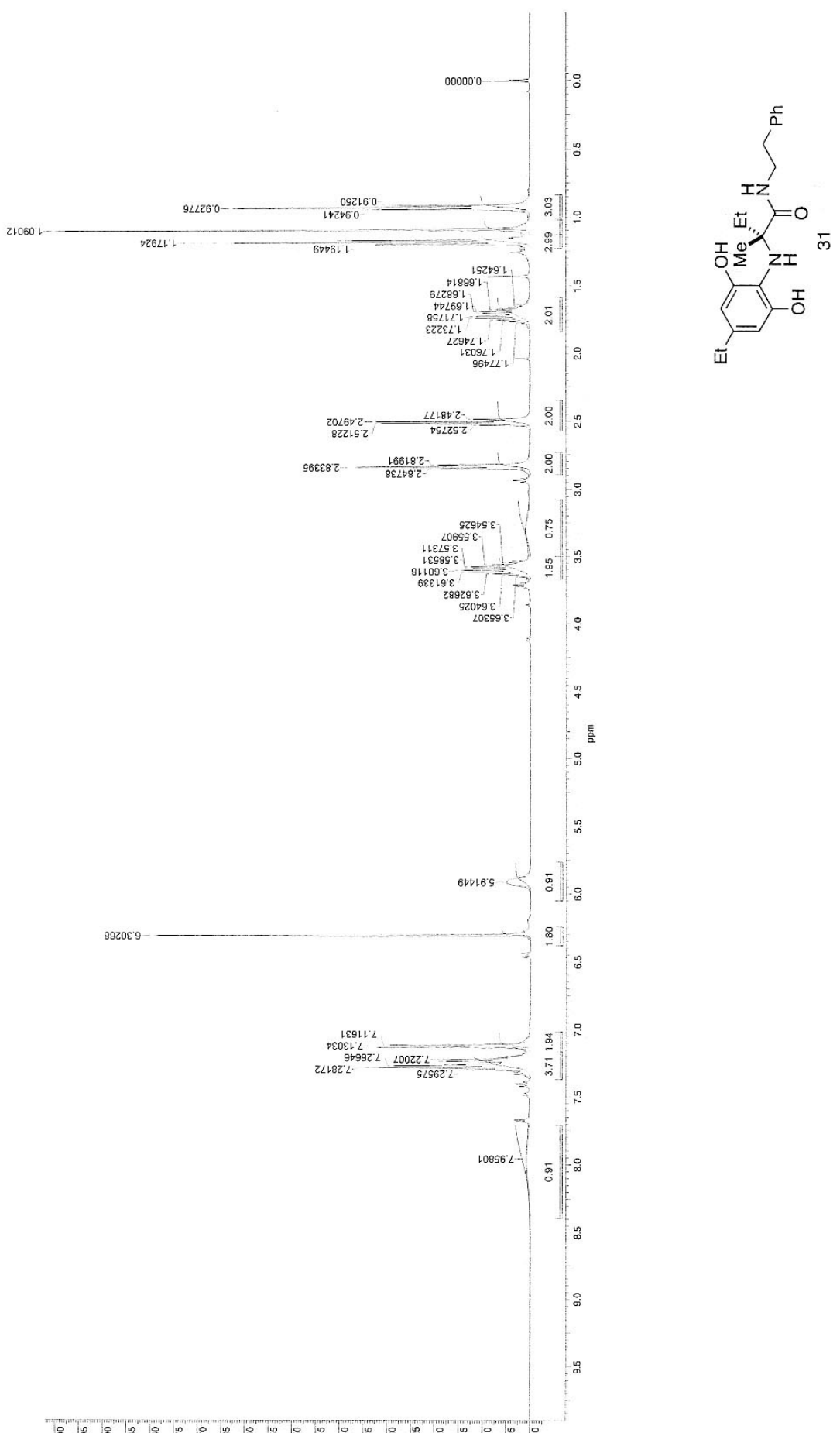

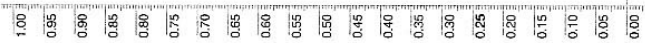




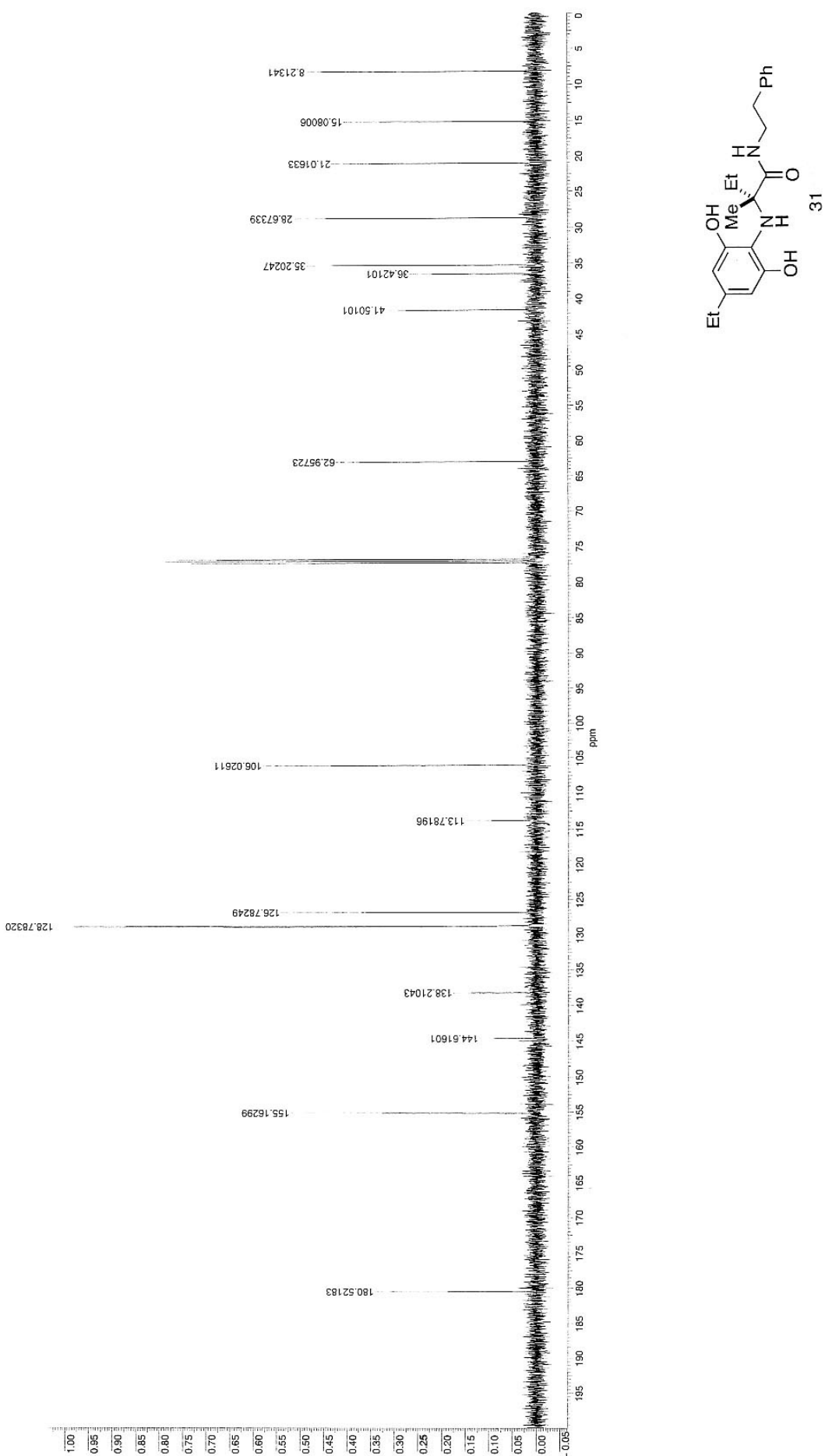




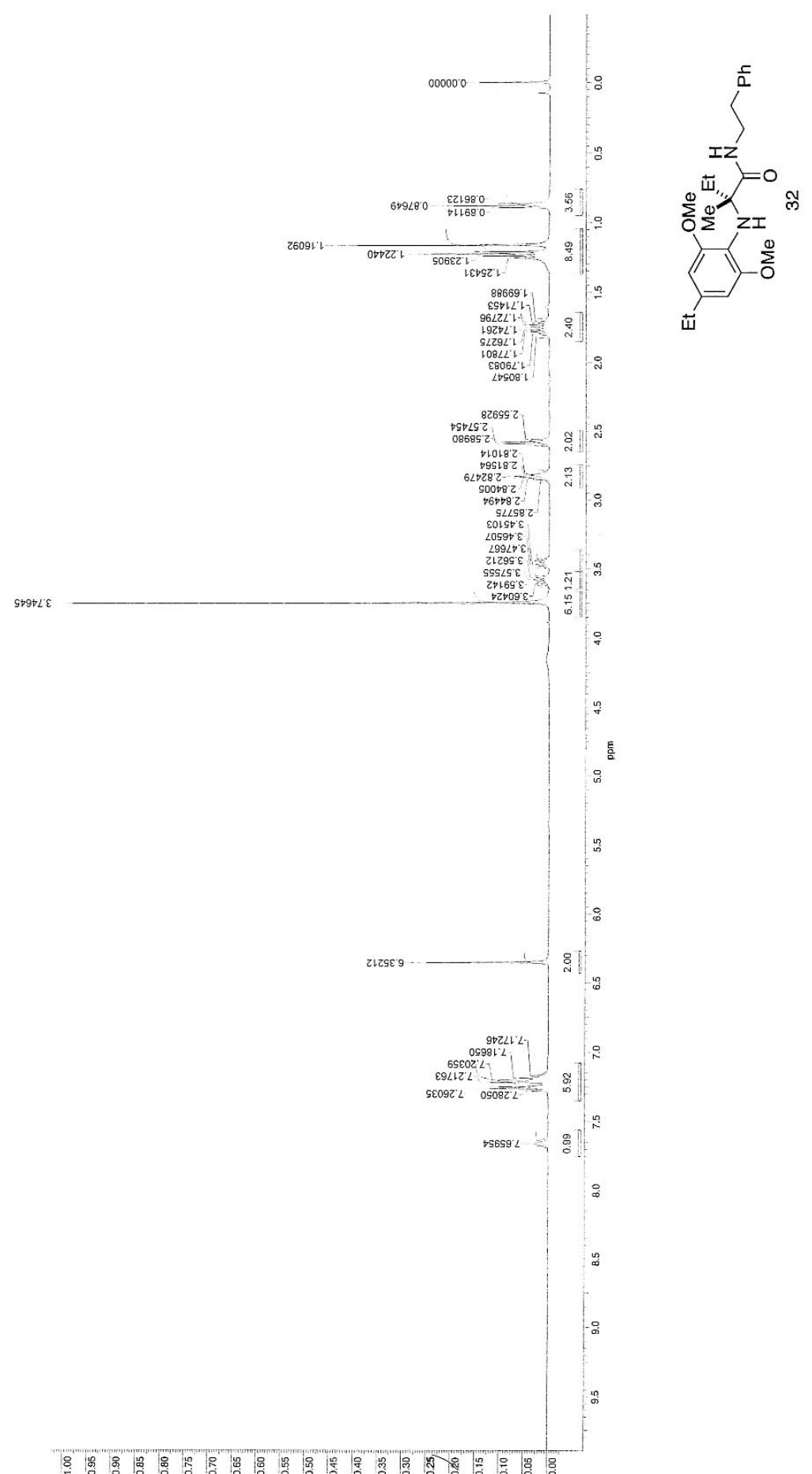




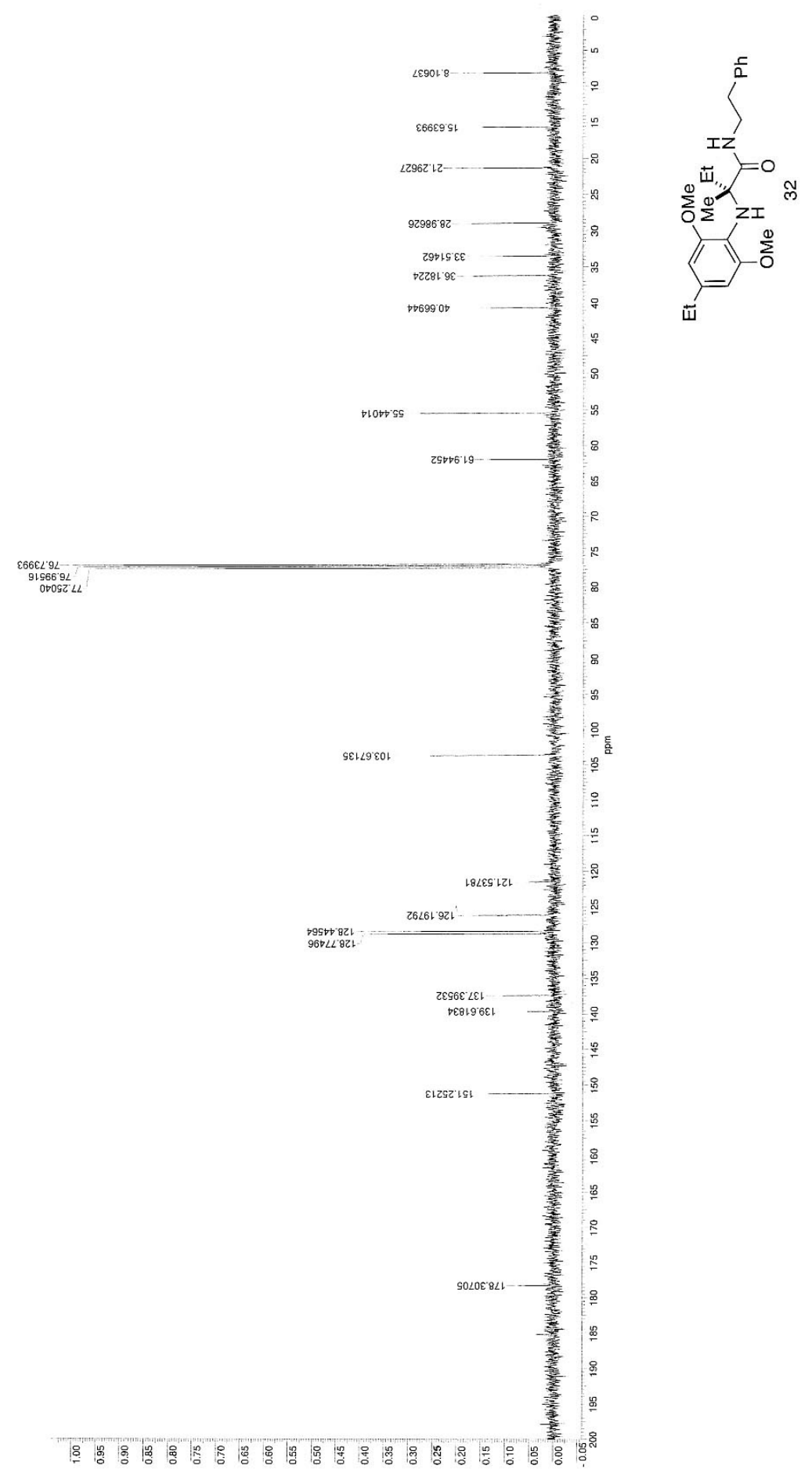




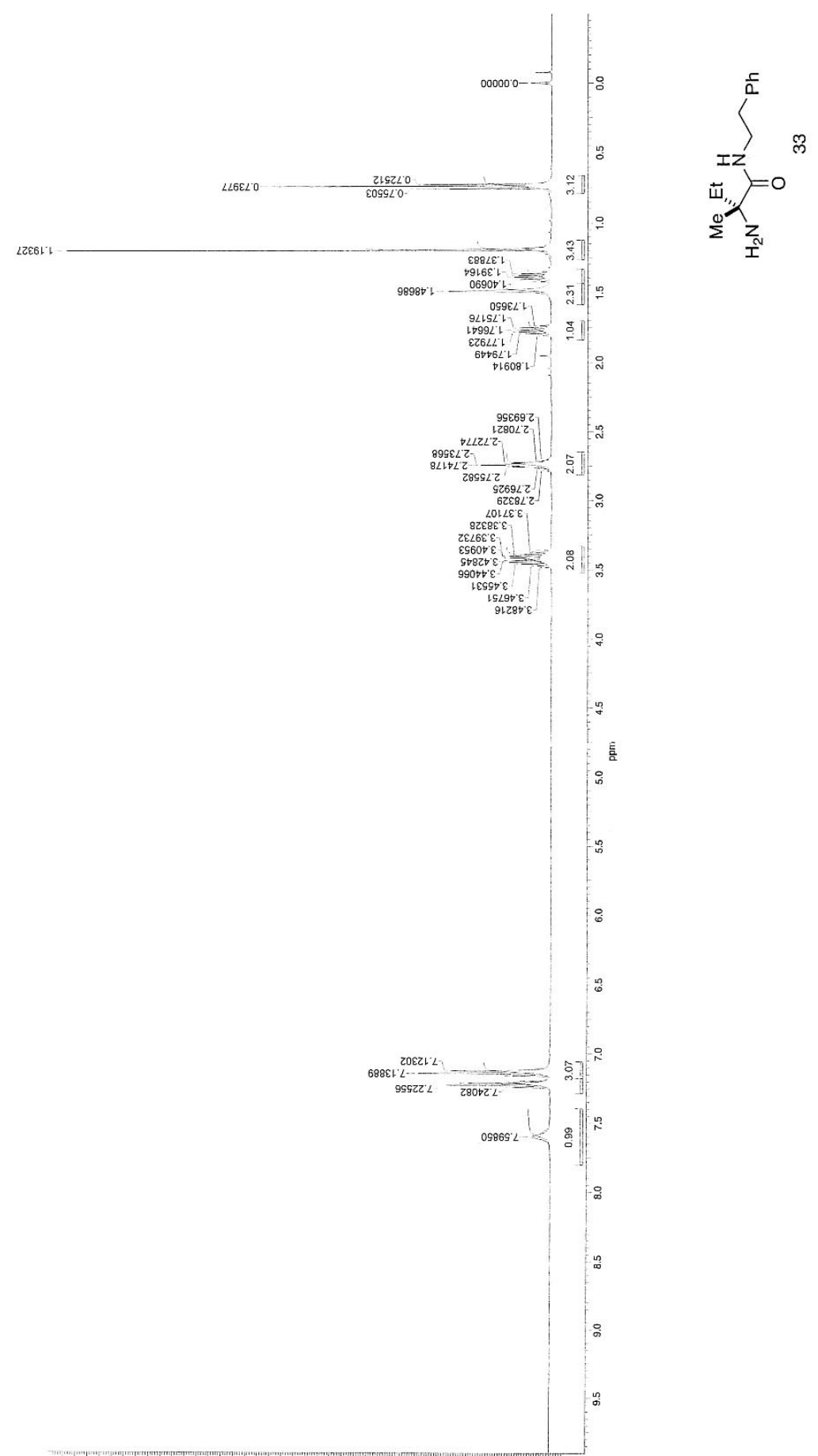

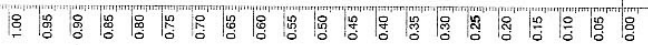




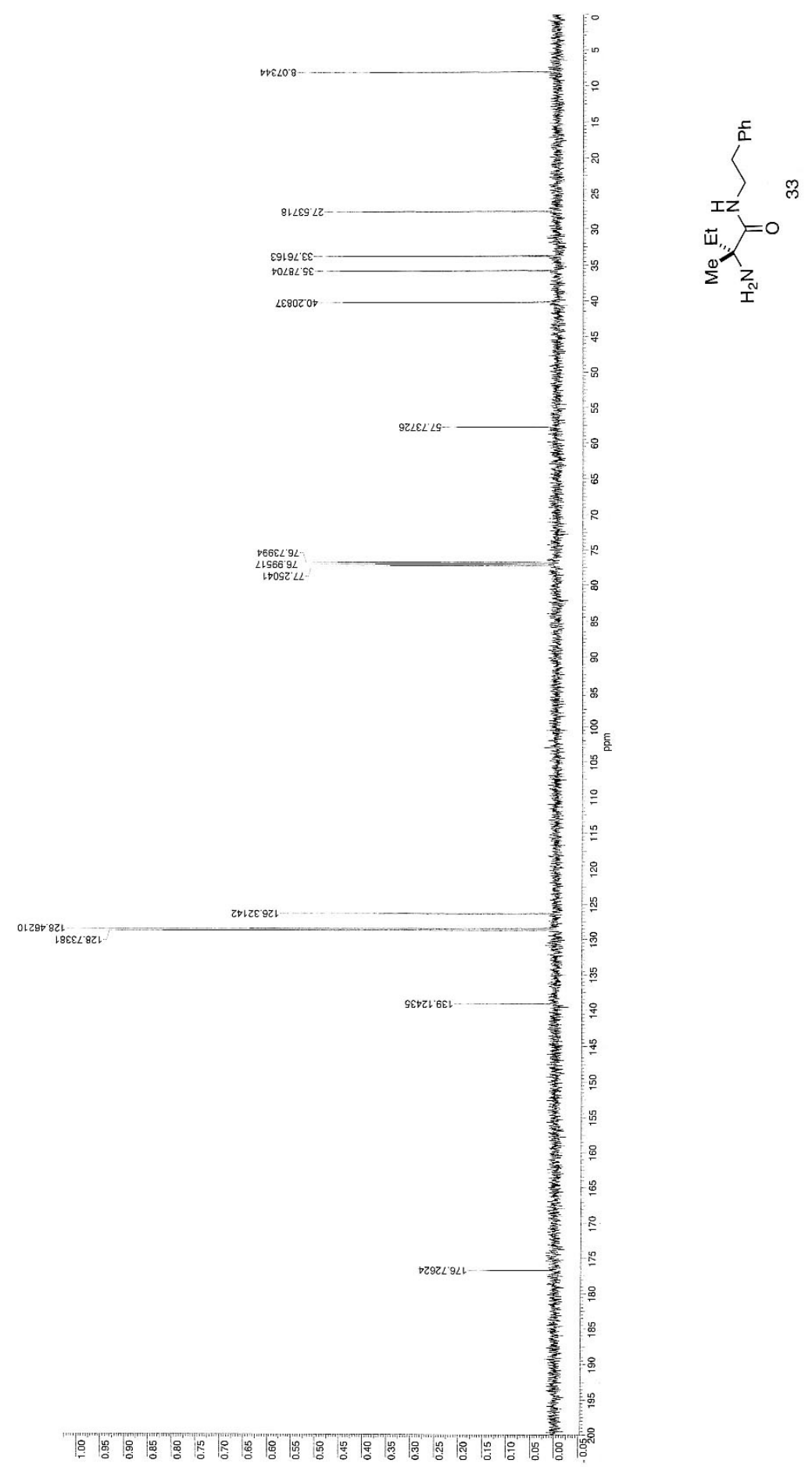




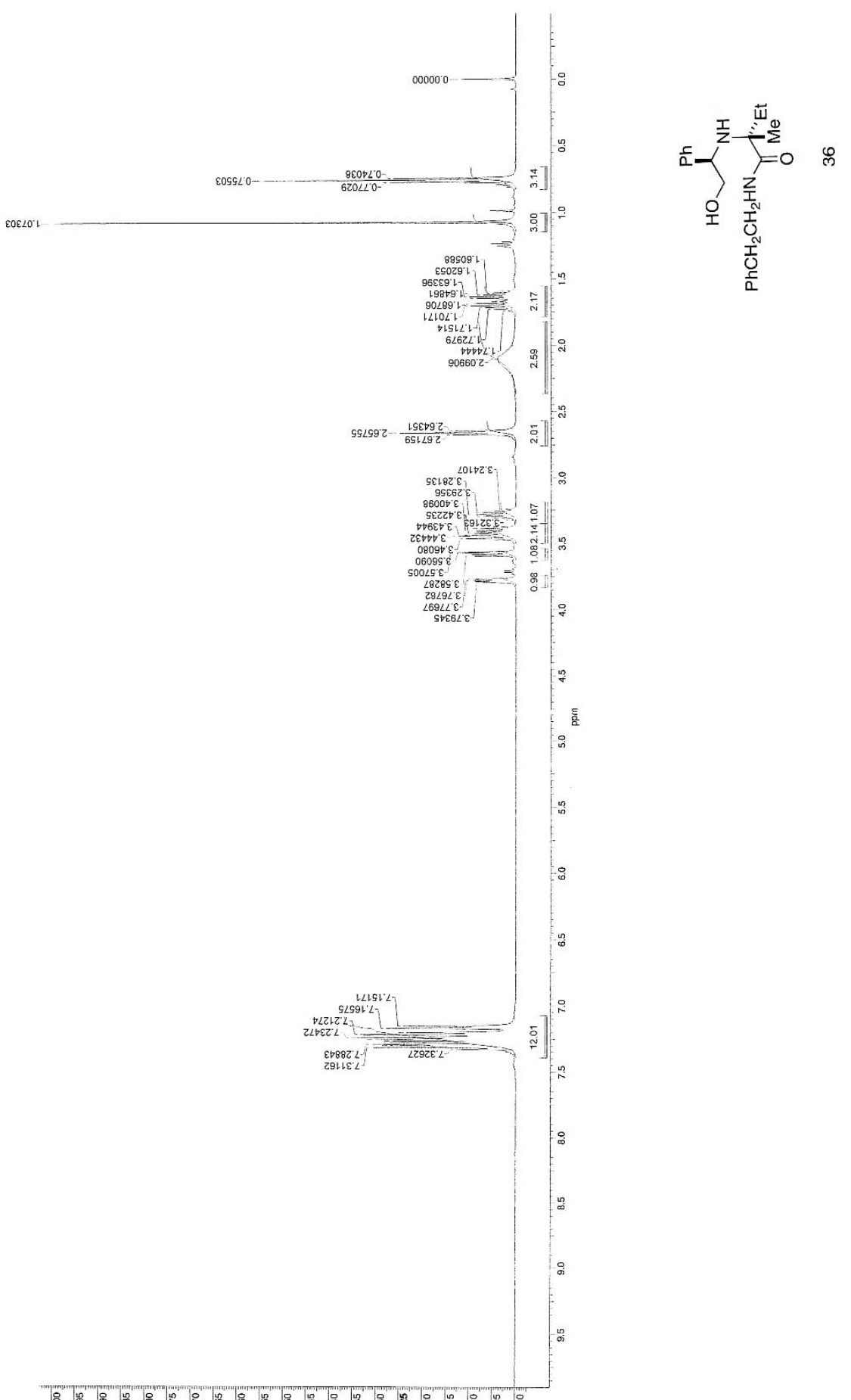

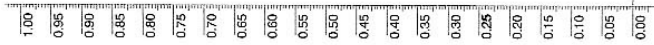




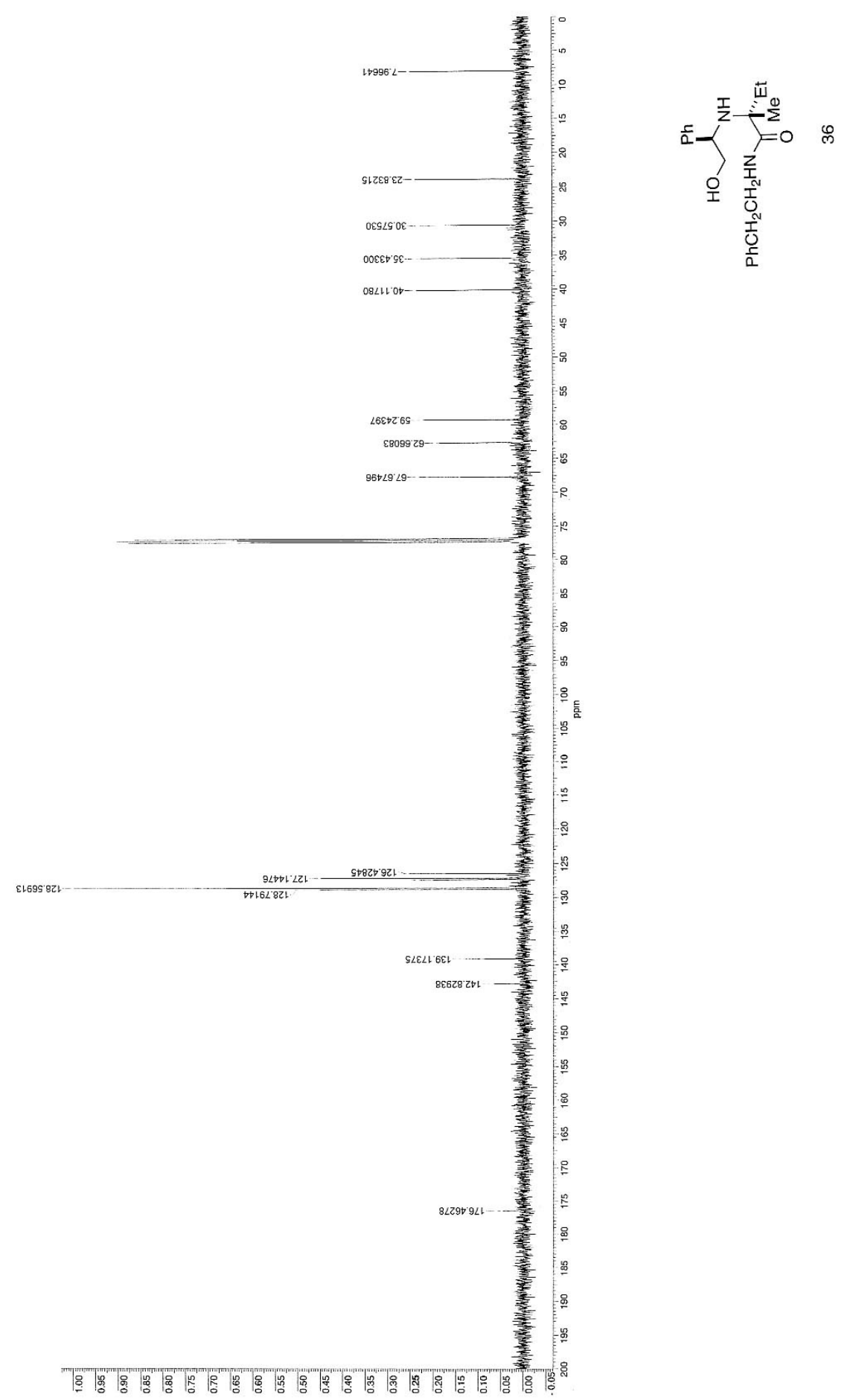

S95 


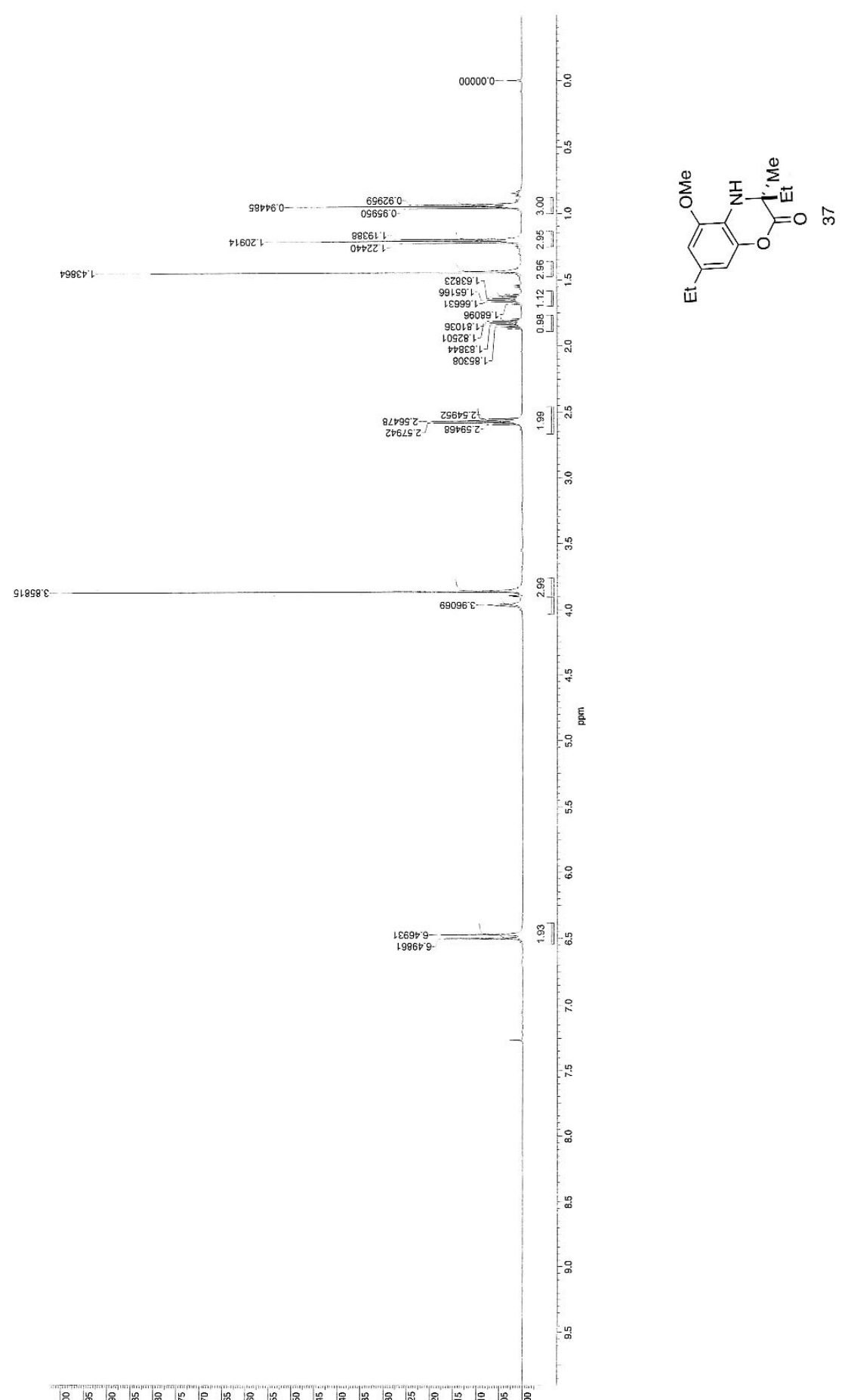

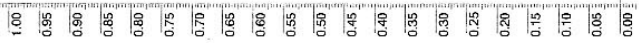




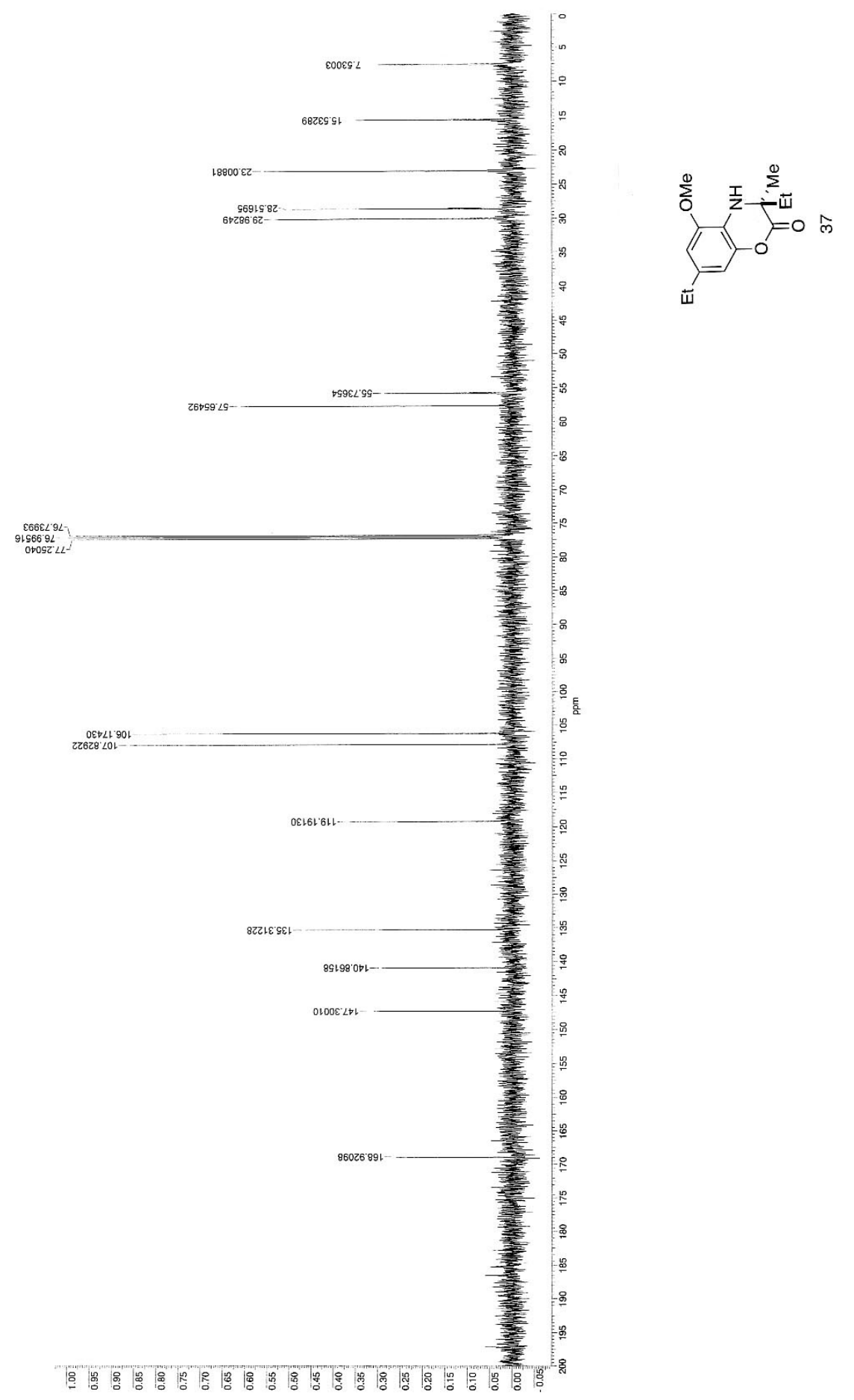




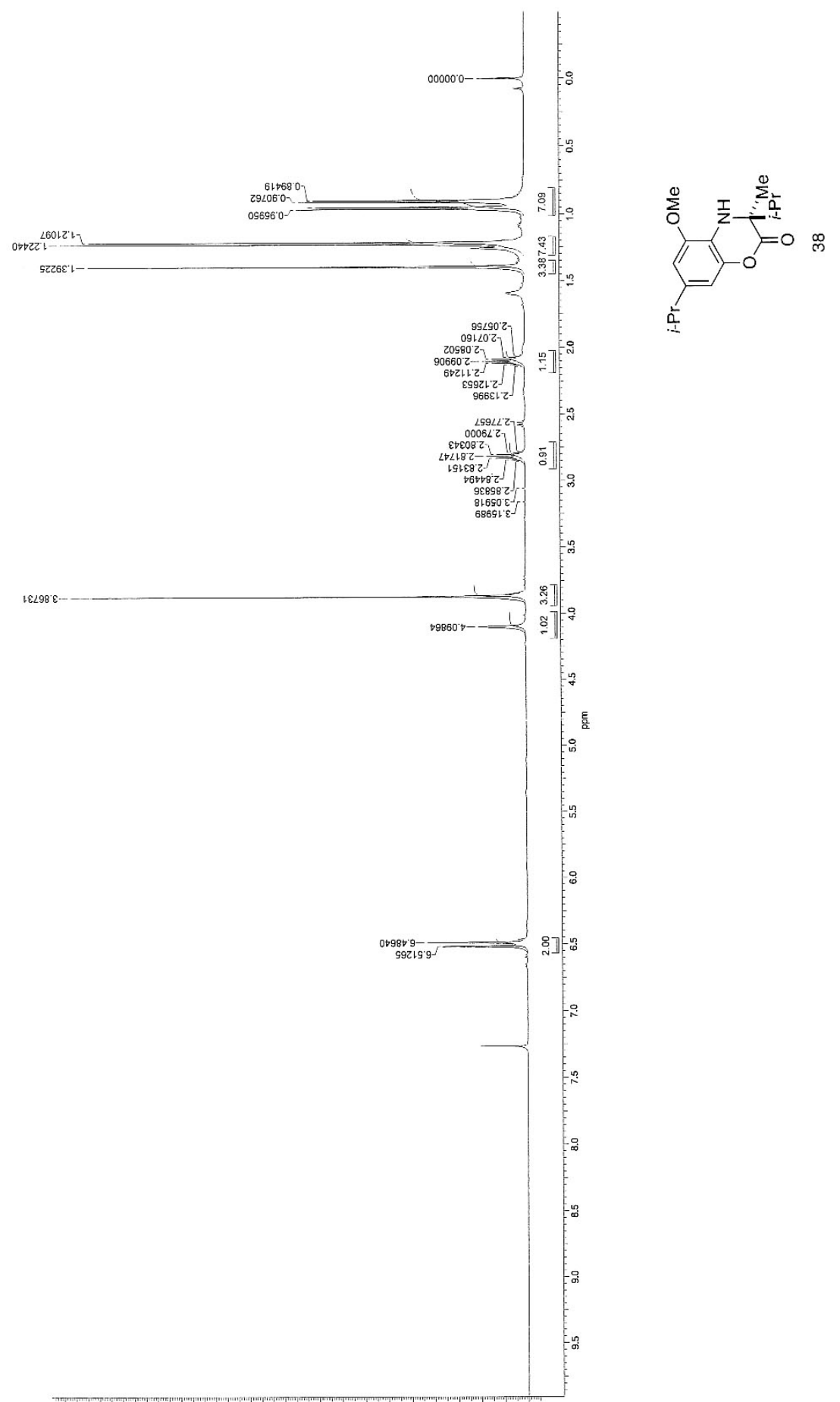

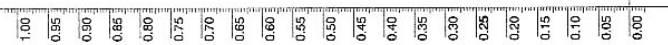




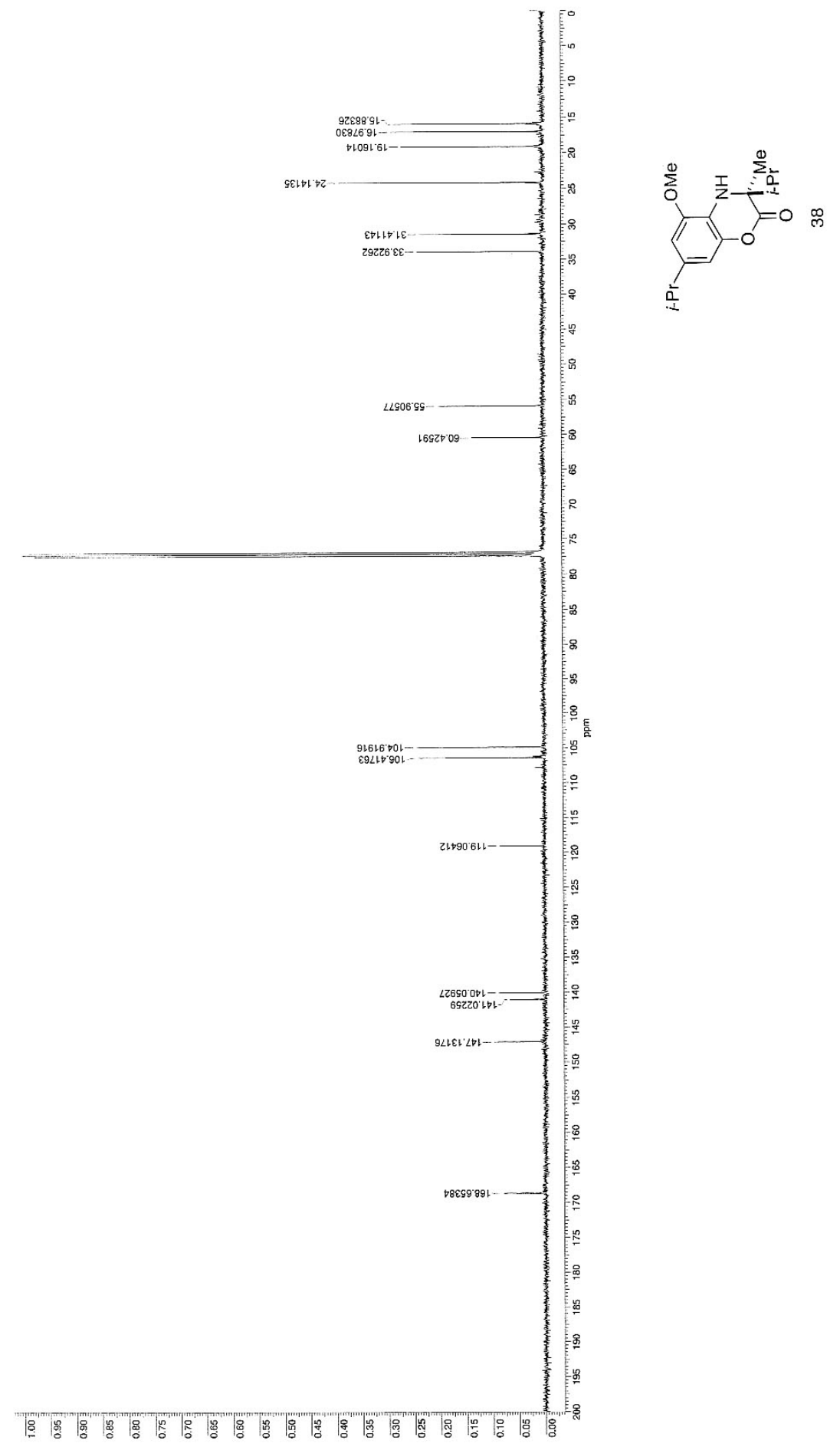

